\title{
CANONICAL BASES \\ ARISING FROM QUANTIZED ENVELOPING ALGEBRAS
}

\author{
G. LUSZTIG
}

\section{INTRODUCTION}

0.1 . Let $\mathbf{U}^{+}$be the + part of the quantized enveloping algebra $\mathbf{U}$ associated by Drinfeld and Jimbo to a root system. This is an algebra over the field of rational functions $\mathbf{Q}(v)$ which for $v=1$ specializes to the classical enveloping algebra $\mathbf{U}_{1}^{+}$of the nilpotent radical of a Borel subalgebra in a semisimple Lie algebra.

0.2. We are interested in the problem of constructing bases of $\mathbf{U}^{+}$as a $\mathbf{Q}(v)$ vector space. One class of bases of $\mathbf{U}^{+}$has been given in [DL]. We call them (or, rather, a slight modification of them, see $\S 2$ ) bases of PBW type, since for $v=1$, they specialize to bases of $\mathbf{U}_{1}^{+}$of the type provided by the PoincaréBirkhoff-Witt theorem. There are many bases of PBW type, one for each reduced expression of the longest element in the Weyl group.

One of the main results of this paper is the construction of a canonical basis B of $\mathbf{U}^{+}$. (We assume for simplicity that we are in the simply laced case; see however $\S 12$.)

0.3. The definition of $\mathbf{B}$ is as follows (see Theorem 3.2). First one shows that the $\mathbf{Z}\left[v^{-1}\right]$ - submodule generated by any basis of PBW type is independent of the choice of that basis; we denote this submodule by $\mathscr{L}$. Next one shows that the image of any basis of PBW type under the natural map $\pi: \mathscr{L} \rightarrow \mathscr{L} / v^{-1} \mathscr{L}$ is a $\mathrm{Z}$-basis of $\mathscr{L} / v^{-1} \mathscr{L}$ that is independent of the choice of the basis of PBW type; we denote this $\mathbf{Z}$-basis by $B$. Finally, one considers the $\mathbf{Q}$-algebra involution ${ }^{-}: \mathbf{U}^{+} \rightarrow \mathbf{U}^{+}$that is the identity on the canonical $\mathbf{Q}(v)$-algebra generators and takes $v$ to $v^{-1}$; one shows that there is a unique $\mathbf{Z}\left[v^{-1}\right]$-basis B of $\mathscr{L}$ such that each element of $\mathbf{B}$ is fixed by ${ }^{-}$and is such that $\pi(\mathbf{B})=B$.

The first two steps in this definition are elementary (see $\S 2$ ); the final step is less so, in the sense that to establish it, we must use some results from the representation theory of quivers. In more detail, it follows from Gabriel's theorem $[G, B G P]$ that a basis of PBW type of $\mathbf{U}_{1}^{+}$should be naturally parametrized

Received by the editors January 22, 1990.

1980 Mathematics Subject Classification (1985 Revision). Primary 20 G99.

This research was supported in part by National Foundation Grant DMS 8702842. 
by isomorphism classes of finite-dimensional "representations" of a quiver (the Dynkin graph with a chosen orientation); moreover, Ringel [R] has made the remarkable observation that the multiplication in $\mathbf{U}_{1}^{+}$and $\mathbf{U}^{+}$can be interpreted in terms of representations of quivers (over a finite field). (We give an exposition of the representation theory of quivers in $\S \S 4,5$.) Now the representations of fixed dimension of a quiver may be viewed as points of some vector space with an algebraic group action so that two points are in the same orbit precisely when the corresponding representations are isomorphic. The dimension of these orbits (or strata) can be computed explicitly (see $\S 6$ ) and this is used in establishing Theorem 3.2 (see $\S 7$ ).

Notice the analogy between the definition of $\mathbf{B}$ and the definition of the new basis for a Hecke algebra given in [KL1].

0.4. One of the main observations of this paper is that Ringel's interpretation of the multiplication in $\mathbf{U}^{+}$can be reformulated in purely geometric terms, using a "convolution" operation on complexes in the derived category of constructible sheaves that are constant on the strata mentioned in 0.3 (over an algebraically closed field). This allows us to use the theory of perverse sheaves.

Consider the Zariski closure of one of the strata mentioned in 0.3. One of our results is a description of the local intersection cohomology of such a closure. Namely, we prove $($ see $\S \S 9,10)$ that this local intersection cohomology vanishes in odd degrees and that its Poincare polynomial at any point in a lower stratum is equal to a certain entry of the transition matrix between the basis $\mathbf{B}$ and a basis of PBW type of $\mathbf{U}^{+}$associated to the orientation. This shows that this Poincare polynomial is (in principle) computable. Notice the analogy between these results and those of [KL2], which concerned Schubert varieties.

0.5 . In $\S 11$ we consider the problem analogous to that in 0.4 , for the "cyclic quiver"; this corresponds to an affine Dynkin graph of type $A$. In this case it is natural to restrict oneself to representations of the quiver that satisfy a certain nilpotency condition, in order to have only finitely many isomorphism classes of representations of a fixed dimension. We show that, in this case, the closures of the corresponding strata are locally isomorphic to affine Schubert varieties of type $A$.

0.6. The canonical basis $\mathbf{B}$ has a number of remarkable properties. One of them is that the product of two elements in $\mathbf{B}$ is a linear combination of elements in B with coefficients in $\mathbf{N}\left[v, v^{-1}\right]$.

Another one is that $\mathbf{B}$ is well adapted to finite-dimensional representations of $\mathbf{U}$. Namely, let $L_{\mathbf{d}}$ be a finite-dimensional simple $\mathbf{U}$-module corresponding to the dominant weight $\mathbf{d}$ and let $x_{0}$ be a lowest weight vector for it. Consider the subset of $\mathbf{B}$ consisting of all elements $\mathscr{E} \in \mathbf{B}$ such that $\mathscr{E} x_{0} \neq 0$. We will show in $\S 8$ that the elements $\mathscr{E} x_{0}$ with $\mathscr{E}$ running through this subset form a basis $\mathbf{B}[\mathbf{d}]$ of $L_{\mathbf{d}}$. This is a canonical basis of $L_{\mathbf{d}}$ that appears to have extremely favorable properties. It gives rise to a canonical basis in any finitedimensional simple module of the corresponding semisimple Lie algebra that, for type $A$, should be closely related to the basis in [DK]. 
0.7. In 8.13 we give a purely combinatorial formula for $\operatorname{dim} L_{\mathbf{d}}$, in the spirit of a conjecture in [BZ]. An analogous formula holds for the individual weight spaces of $L_{\mathbf{d}}$. We will show elsewhere that there is a canonical basis for the space of invariants in the tensor product of three modules of form $L_{\mathbf{d}}$, and that the dimension of this space is given by a purely combinatorial formula. (This follows easily from the results of this paper.)

\section{CONTENTS}

1. Notations.

2. Definition of the subquotient $\mathscr{L} / v^{-1} \mathscr{L}$ of $U^{+}$.

3. Definition of the canonical basis $\mathbf{B}$.

4. Quivers.

5. Multiplication.

6. Dimension of orbits.

7. A formula for $f_{\mathbf{c}}$.

8. Finite-dimensional U-modules.

9. Intersection cohomology.

10. Purity.

11. The cyclic quiver.

12. Comments on the non-simply laced case.

13. Comments on Fourier transform.

\section{Notations}

1.1. Let $v$ be an indeterminate, let $A=\mathbf{Z}\left[v, v^{-1}\right]$ and let $A^{\prime}=\mathbf{Q}(v)$ be its quotient field. Let ${ }^{-}: A^{\prime} \rightarrow A^{\prime}$ be the field automorphism (of order two) that takes $v$ to $v^{-1}$; it restricts to an automorphism of the ring $A$. Given integers $N, M \geq 0$ we define

$$
[N] !=\prod_{h=1}^{N} \frac{v^{h}-v^{-h}}{v-v^{-1}} \in A, \quad\left[\begin{array}{c}
M+N \\
N
\end{array}\right]=\frac{[M+N] !}{[M] ![N] !} \in A .
$$

1.2. Let $\left(a_{i j}\right)_{1 \leq i, j \leq n}$ be a symmetric, positive definite Cartan matrix. Let $Q$ be the free abelian group with basis $\left\{\alpha_{1}, \ldots, \alpha_{n}\right\}$. Define an inner product (, ) on $Q$ by $\left(\alpha_{i}, \alpha_{j}\right)=a_{i j}$. Let $R=\{\alpha \in Q \mid(\alpha, \alpha)=2\}$. This is a root system whose set of simple roots is $\left\{\alpha_{1}, \ldots, \alpha_{n}\right\}$. Let $R^{+}=\{\alpha \in R \mid \alpha=$ $\left.\sum c_{j} \alpha_{j}, c_{j} \in \mathbf{N}\right\}, R^{-}=-R^{+}$. Any $\alpha \in R$ defines a reflection $s_{\alpha}: Q \rightarrow Q$, $z \rightarrow z-(z, \alpha) \alpha$. We write $s_{i}$ instead of $s_{\alpha_{i}}$. Let $W$ be the Weyl group of $R$; it is the subgroup of $\operatorname{Aut}(Q)$ generated by the reflections $s_{i}(1 \leq i \leq n)$. Let $l(w)$ be the usual length function on $W$ with respect to the generators $\left\{s_{1}, \ldots, s_{n}\right\}$. Let $\nu=\# R^{+}$. Let $w_{0}$ be the unique element of $W$ of maximal length $(=\nu)$.

Let $\mathbf{U}$ be the Drinfeld-Jimbo quantized enveloping algebra corresponding to $\left(a_{i j}\right)$. With the notation of $[\mathrm{L} 1]$, this is the $A^{\prime}$-algebra with generators 
$E_{i}, F_{i}, K_{i}, K_{i}^{-1}(1 \leq i \leq n)$ and relations

$$
\begin{aligned}
& K_{i} K_{j}=K_{j} K_{i}, \quad K_{i} K_{i}^{-1}=1, \\
& K_{i} E_{j}=v^{a_{i j}} E_{j} K_{i}, \quad K_{i} F_{j}=v^{-a_{i j}} F_{j} K_{i}, \\
& E_{i} F_{j}-F_{j} E_{i}=\delta_{i j} \frac{K_{i}-K_{i}^{-1}}{v-v^{-1}}, \\
& \left\{\begin{array}{c}
E_{i}^{2} E_{j}-\left(v+v^{-1}\right) E_{i} E_{j} E_{i}+E_{j} E_{i}^{2}=0 \quad \text { if } a_{i j}=-1, \\
E_{i} E_{j}=E_{j} E_{i} \quad \text { if } a_{i j}=0,
\end{array}\right. \\
& \left\{\begin{array}{c}
F_{i}^{2} F_{j}-\left(v+v^{-1}\right) F_{i} F_{j} F_{i}+F_{j} F_{i}^{2}=0 \text { if } a_{i j}=-1, \\
F_{i} F_{j}=F_{j} F_{i} \text { if } a_{i j}=0 .
\end{array}\right.
\end{aligned}
$$

Let $\mathbf{U}^{+}$be the $A^{\prime}$-subalgebra of $\mathbf{U}$ generated by the elements $E_{i}(1 \leq i \leq n)$; it may be identified with the $A^{\prime}$-algebra defined by the generators $E_{i}(1 \leq i \leq n)$ and relations (a4). Let $\mathbf{U}^{0}$ be the $A^{\prime}$-subalgebra of $\mathbf{U}$ generated by the elements $K_{i}, K_{i}^{-1}(1 \leq i \leq n)$; it may be identified with the $A^{\prime}$-algebra defined by the generators $K_{i}, K_{i}^{-1}(1 \leq i \leq n)$ and relations (a1). Let $U$ (resp. $U^{+}$) be the $A$-subalgebra of $\mathrm{U}$ (resp. $\mathrm{U}^{+}$) generated by the elements

$$
E_{i}^{(N)}=E_{i}^{N} /[N] !, \quad F_{i}^{(N)}=F_{i}^{N} /[N] !, \quad K_{i}, \quad K_{i}^{-1}
$$

(resp. $E_{i}^{(N)}$ ) for $1 \leq i \leq n, N \geq 0$.

Let ${ }^{-}: \mathbf{U} \rightarrow \mathbf{U}$ be the $\mathbf{Q}$-algebra involution defined by

$$
E_{j} \rightarrow E_{j}, \quad F_{j} \rightarrow F_{j}, \quad K_{j} \rightarrow K_{j}^{-1}
$$

for all $j$ and $v \rightarrow v^{-1}$. This maps $\mathbf{U}^{+}, U, U^{+}$into themselves.

1.3. For $i \in[1, n]$, let $T_{i}: \mathbf{U} \rightarrow \mathbf{U}$ be the $A^{\prime}$-algebra automorphism defined in [L1,1.3]. Its inverse $T_{i}^{-1}$ is given by

$$
\begin{aligned}
E_{j} \rightarrow-K_{j}^{-1} F_{j}, & F_{j} \rightarrow-E_{j} K_{j}, \quad K_{j} \rightarrow K_{j}^{-1} \quad \text { if } i=j, \\
E_{j} \rightarrow E_{j}, & F_{j} \rightarrow F_{j}, \quad K_{j} \rightarrow K_{j} \quad \text { if } a_{i j}=0, \\
E_{j} \rightarrow-E_{j} E_{i}+v^{-1} E_{i} E_{j}, & F_{j} \rightarrow-F_{i} F_{j}+v F_{j} F_{i}, \quad K_{j} \rightarrow K_{i} K_{j} \quad \text { if } a_{i j}=-1 .
\end{aligned}
$$

We have the braid group relations:

$$
\left\{\begin{array}{c}
T_{i}^{-1} T_{j}^{-1} T_{i}^{-1}=T_{j}^{-1} T_{i}^{-1} T_{j}^{-1} \quad \text { if } a_{i j}=-1, \\
T_{i}^{-1} T_{j}^{-1}=T_{j}^{-1} T_{i}^{-1} \quad \text { if } a_{i j}=0 .
\end{array}\right.
$$

Let $r_{i}: \mathbf{U} \rightarrow \mathbf{U}$ be the $A^{\prime}$-algebra automorphism defined by

$$
E_{j} \rightarrow(-1)^{a_{i j}} E_{j}, \quad F_{j} \rightarrow(-1)^{a_{i j}} F_{j}, \quad K_{j} \rightarrow K_{j} .
$$


Let $\tilde{T}_{i}: \mathbf{U} \rightarrow \mathbf{U}$ be the composition $T_{i}^{-1} r_{i}$. We have

$$
\tilde{T}_{i} \tilde{T}_{j} \tilde{T}_{j}=T_{i}^{-1} T_{j}^{-1} T_{i}^{-1} \quad \text { if } a_{i j}=-1, \quad \widetilde{T}_{i} \widetilde{T}_{j}=T_{i}^{-1} T_{j}^{-1} r_{i} r_{j} \quad \text { if } a_{i j}=0 .
$$

Hence

$$
\tilde{T}_{i} \tilde{T}_{j} \tilde{T}_{i}=\tilde{T}_{j} \tilde{T}_{i} \widetilde{T}_{j} \quad \text { if } a_{i j}=-1, \quad \tilde{T}_{i} \widetilde{T}_{j}=\tilde{T}_{j} \tilde{T}_{i} \quad \text { if } a_{i j}=0
$$

Just as in $[\mathrm{L} 1,1.8(\mathrm{~d})]$ we see that the following holds.

(c) If $s_{i_{1}} \cdots s_{i_{k}}$ is a reduced expression in $W$ and $s_{i_{1}} \cdots s_{i_{k-1}}\left(\alpha_{i_{k}}\right)=\alpha_{j}$ for some $j \in[1, n]$ then $\widetilde{T}_{i_{1}} \cdots \widetilde{T}_{i_{k-1}}\left(E_{i_{k}}\right)=E_{j}$.

1.4. There is a unique $A^{\prime}$-algebra isomorphism $\Psi: \mathbf{U} \cong \mathbf{U}^{\text {opp }}$ such that $E_{i} \rightarrow$ $E_{i}, F_{i} \rightarrow F_{i}, K_{i} \rightarrow K_{i}^{-1}$. We have $\Psi \widetilde{T}_{i}=\widetilde{T}_{i}^{-1} \Psi$ for all $i$.

\section{Definition of the SUbQuotient $\mathscr{L} / v^{-1} \mathscr{L}$ of $U^{+}$AND ITS Basis $B$}

2.1. Let $\mathscr{X}$ be the set of all sequences $\mathbf{i}=\left(i_{1}, \ldots, i_{\nu}\right)$ in $[1, n]$ such that $s_{i_{1}} \cdots s_{i_{\nu}}=w_{0}$.

We shall regard $\mathscr{X}$ as the set of vertices of a graph $\mathbf{X}$ in which $\mathbf{i}=$ $\left(i_{1}, \ldots, i_{\nu}\right)$ and $\mathbf{i}^{\prime}=\left(i_{1}^{\prime}, \ldots, i_{\nu}^{\prime}\right)$ are joined if $\mathbf{i}^{\prime}$ is obtaining from $\mathbf{i}$ by

(a) replacing three consecutive entries $h, k, h$ in $\mathbf{i}$ (with $a_{h k}=-1$ ) by $k, h, k$ or by

(b) replacing two consecutive entries $h, k$ in $\mathbf{i}$ (with $a_{h k}=0$ ) by $k, h$.

For such joined $\left(\mathbf{i}, \mathbf{i}^{\prime}\right)$, i.e., in case (a) (resp. (b)), we define a map $R_{\mathbf{i}}^{\mathbf{i}^{\prime}}: \mathbf{N}^{\nu} \cong$ $\mathbf{N}^{\nu}$ as follows. This map takes $\mathbf{c}=\left(c_{1}, \ldots, c_{\nu}\right) \in \mathbf{N}^{\nu}$ to $\mathbf{c}^{\prime}=\left(c_{1}^{\prime}, \ldots, c_{\nu}^{\prime}\right) \in$ $\mathbf{N}^{\nu}$, which has the same coordinates as $\mathbf{c}$ except in the three (resp. two) consecutive positions at which $\left(\mathbf{i}, \mathbf{i}^{\prime}\right)$ differ; if $(a, b, c)$ (resp. $\left.(a, b)\right)$ are the coordinates of $\mathbf{c}$ at those three (resp. two) positions, the coordinates of $\mathbf{c}^{\prime}$ at those positions are

$$
(b+c-\min (a, c), \min (a, c), a+b-\min (a, c)) \quad(\text { resp. }(b, a)) .
$$

It is easy to check that $R_{\mathrm{i}}^{\mathbf{i}^{\prime}}$ is a bijection; its inverse is $R_{\mathbf{i}^{\prime}}^{\mathbf{i}}$.

It is well known that

(c) the graph $\mathbf{X}$ is connected.

We now define a new graph $\tilde{\mathbf{X}}$. The vertices of $\tilde{\mathbf{X}}$ are the pairs $(\mathbf{i}, \mathbf{c}) \in$ $\mathscr{X} \times \mathbf{N}^{\nu}$. Two such pairs $(\mathbf{i}, \mathbf{c}),\left(\mathbf{i}^{\prime}, \mathbf{c}^{\prime}\right)$ are joined precisely when $\left(\mathbf{i}, \mathbf{i}^{\prime}\right)$ are joined in $\mathbf{X}$ and $R_{\mathbf{i}}^{\mathbf{i}^{\prime}}(\mathbf{c})=\mathbf{c}^{\prime}$.

We have a morphism of graphs $p r_{1}: \tilde{\mathbf{X}} \rightarrow \mathbf{X}, \quad(\mathbf{i}, \mathbf{c}) \rightarrow \mathbf{i}$. This is a covering map between the corresponding 1-dimensional simplicial complexes.

2.2. Given $\mathbf{i}=\left(i_{1}, \ldots, i_{\nu}\right) \in \mathscr{X}$ and $\mathbf{c}=\left(c_{1}, \ldots, c_{\nu}\right) \in \mathbf{N}^{\nu}$ we define

$$
E_{\mathbf{i}}^{\mathbf{c}}=E_{i_{1}}^{\left(c_{1}\right)} \tilde{T}_{i_{1}}\left(E_{i_{2}}^{\left(c_{2}\right)}\right) \tilde{T}_{i_{1}} \tilde{T}_{i_{2}}\left(E_{i_{3}}^{\left(c_{3}\right)}\right) \cdots \tilde{T}_{i_{1}} \tilde{T}_{i_{2}} \cdots \tilde{T}_{i_{\nu-1}}\left(E_{i_{\nu}}^{\left(c_{\nu}\right)}\right) \text {. }
$$

According to $[\mathrm{L} 1,1.8,1.13]$, for any $\mathbf{i} \in \mathscr{X}$, the elements $E_{\mathbf{i}}^{\mathbf{c}} \quad\left(\mathbf{c} \in \mathbf{N}^{\nu}\right)$ are contained in $U^{+}$and form an $A^{\prime}$-basis of $\mathbf{U}^{+}$. (We shall denote this basis by $B_{\mathbf{i}}$ ) 
Hence, given $\mathbf{i}, \mathbf{i}^{\prime} \in \mathscr{Z}$ and $\mathbf{c} \in \mathbf{N}^{\nu}$, we can write uniquely

$$
E_{\mathbf{i}}^{\mathbf{c}}=\sum_{\mathbf{c}^{\prime} \in \mathbf{N}^{\nu}} \gamma_{\mathbf{i}, \mathbf{i}^{\prime}}^{\mathbf{c}, \mathbf{c}^{\prime}} E_{\mathbf{i}^{\prime}}^{\mathbf{c}^{\prime}}
$$

where $\gamma_{\mathbf{i}, \mathbf{i}^{\prime}}^{\mathbf{c}, \mathbf{c}^{\prime}} \in A^{\prime}$

Proposition 2.3. Let $\mathbf{i} \in \mathscr{X}$. (a) $B_{\mathbf{i}}$ is a basis of $U^{+}$as an A-module.

(b) Let $\mathscr{L}_{\mathbf{i}}$ be the $\mathrm{Z}\left[v^{-1}\right]$-submodule of $\mathbf{U}^{+}$generated by the basis $B_{\mathrm{i}}$. Then $\mathscr{L}_{\mathbf{i}}$ is independent of $\mathbf{i} \in \mathscr{Z}$. We denote it $\mathscr{L}$.

(c) Let $\pi: \mathscr{L} \rightarrow \mathscr{L} / v^{-1} \mathscr{L}$ be the canonical projection. Then $\pi\left(B_{\mathrm{i}}\right)$ is a Z-basis of $\mathscr{L} / v^{-1} \mathscr{L}$, independent of $\mathbf{i} \in \mathscr{X}$; we denote it $B$.

(d) Assume that $\mathbf{i}, \mathbf{i}^{\prime} \in \mathscr{X}$ are joined in the graph $\mathbf{X}$. For $\mathbf{c}, \mathbf{c}^{\prime} \in \mathbf{N}^{\nu}, \gamma_{\mathbf{i}, \mathbf{i}^{\prime}}^{\mathbf{c}, \mathbf{c}^{\prime}}$ is in $\mathbf{Z}\left[v^{-1}\right]$. Its constant term is 1 if $\mathbf{c}^{\prime}=R_{\mathbf{i}}^{\mathrm{i}^{\prime}}(\mathbf{c})$ and is zero otherwise.

The proof is a refinement of arguments in [DL], where (a) (or a statement very close to it) is proved; we shall reprove it here.

Assume that (d) holds. To prove that the objects defined in (b),(c) in terms of $\mathbf{i}, \mathbf{i}^{\prime} \in \mathscr{X}$ coincide, we may assume, in view of $2.1(\mathrm{c})$, that $\mathbf{i}, \mathbf{i}^{\prime}$ are joined in $\mathbf{X}$ in which case the desired statements follow immediately from (d). We can now complete the proof of (a) as in [loc. cit.]. We must show that $A \mathscr{L}=U^{+}$. It is enough to prove that, for any $i, N, A \mathscr{L}$ is stable under left multiplication by $E_{i}^{(N)}$. But if we choose (as we may) $\mathbf{i} \in \mathscr{X}$ with first entry $i$, then $A \mathscr{L}_{\mathbf{i}}$ is clearly stable under left multiplication by $E_{i}^{(N)}$. Since $A \mathscr{L}_{\mathbf{i}}=A \mathscr{L}$ by (b), we see that (a) holds.

It remains to prove (d). Using the definitions and 1.3(b) we see that it is enough to verify (d) under the assumption that $n=2$. In the remainder of this proof we shall therefore assume that $n=2$. The case where $a_{12}=0$ is trivial; we shall therefore assume that $a_{12}=-1$.

In this case we set $\mathbf{i}=(1,2,1), \mathbf{i}^{\prime}=(2,1,2)$. We have

$$
E_{\mathbf{i}}^{a, b, c}=E_{1}^{(a)}[b] !^{-1}\left(E_{2} E_{1}-v^{-1} E_{1} E_{2}\right)^{b} E_{2}^{(c)}=E_{1}^{(a)} \widetilde{T}_{1}\left(E_{2}^{(b)}\right) \widetilde{T}_{1} \widetilde{T}_{2}\left(E_{1}^{(c)}\right)
$$

where $(a, b, c) \in \mathbf{N}^{3}$. Let

$$
D_{b}=[b] !^{-1}\left(-E_{2} E_{1}+v^{-1} E_{1} E_{2}\right)^{b} .
$$
have

Let $\left(m, m^{\prime}, m^{\prime \prime}\right) \in \mathbf{N}^{3}$ be such that $m^{\prime} \geq m+m^{\prime \prime}$. Using [L1, 1.6(b)] we

$$
\begin{aligned}
E_{1}^{(m)} E_{2}^{\left(m^{\prime}\right)} E_{1}^{\left(m^{\prime \prime}\right)} & =E_{1}^{(m)} \sum_{k=0}^{m^{\prime \prime}} v^{-k-\left(m^{\prime}-k\right)\left(m^{\prime \prime}-k\right)} E_{1}^{\left(m^{\prime \prime}-k\right)}(-v)^{k} D_{k} E_{2}^{\left(m^{\prime}-k\right)} \\
& =\sum_{k=0}^{m^{\prime \prime}} v^{-\left(m^{\prime}-k\right)\left(m^{\prime \prime}-k\right)}\left[\begin{array}{c}
m+m^{\prime \prime}-k \\
m
\end{array}\right] E_{\mathrm{i}}^{m+m^{\prime \prime}-k, k, m^{\prime}-k}
\end{aligned}
$$


where

$$
v^{-\left(m^{\prime}-k\right)\left(m^{\prime \prime}-k\right)}\left[\begin{array}{c}
m+m^{\prime \prime}-k \\
m
\end{array}\right] \in v^{-\left(m^{\prime \prime}-k\right)\left(m^{\prime}-m-k\right)}\left(1+v^{-1} \mathbf{Z}\left[v^{-1}\right]\right)
$$

is in $v^{-1} \mathbf{Z}\left[v^{-1}\right]$ if $k<m^{\prime \prime}$ and equals 1 if $k=m^{\prime \prime}$.

Similarly,

$$
\begin{aligned}
E_{2}^{(m)} E_{1}^{\left(m^{\prime}\right)} E_{2}^{\left(m^{\prime \prime}\right)} & =\sum_{k=0}^{m} v^{-(m-k)\left(m^{\prime}-k\right)}\left[\begin{array}{c}
m+m^{\prime \prime}-k \\
m^{\prime \prime}
\end{array}\right] E_{1}^{\left(m^{\prime}-k\right)} D_{k} E_{2}^{\left(m+m^{\prime \prime}-k\right)} \\
& =\sum_{k=0}^{m} v^{-(m-k)\left(m^{\prime}-k\right)}\left[\begin{array}{c}
m+m^{\prime \prime}-k \\
m^{\prime \prime}
\end{array}\right] E_{\mathbf{i}}^{m^{\prime}-k, k, m+m^{\prime \prime}-k}
\end{aligned}
$$

where

$$
v^{-(m-k)\left(m^{\prime}-k\right)}\left[\begin{array}{c}
m+m^{\prime \prime}-k \\
m^{\prime \prime}
\end{array}\right] \in v^{-(m-k)\left(m^{\prime}-m^{\prime \prime}-k\right)}\left(1+v^{-1} \mathbf{Z}\left[v^{-1}\right]\right)
$$

is in $v^{-1} \mathbf{Z}\left[v^{-1}\right]$ if $k<m$ and equals 1 if $k=m$.

Consider the family $\mathscr{F}$ consisting of the following elements of $U^{+}$:

$$
\begin{gathered}
E_{1}^{(m)} E_{2}^{\left(m^{\prime}\right)} E_{1}^{\left(m^{\prime \prime}\right)}, \\
E_{2}^{(m)} E_{1}^{\left(m^{\prime}\right)} E_{2}^{\left(m^{\prime \prime}\right)}
\end{gathered}
$$

for various $\left(m, m^{\prime}, m^{\prime \prime}\right) \in \mathbf{N}^{3}$ such that $m^{\prime} \geq m+m^{\prime \prime}$ where the elements

$$
E_{1}^{(m)} E_{2}^{\left(m+m^{\prime \prime}\right)} E_{1}^{\left(m^{\prime \prime}\right)}=E_{2}^{\left(m^{\prime \prime}\right)} E_{1}^{\left(m+m^{\prime \prime}\right)} E_{2}^{(m)},
$$

which are both of type (e) and (f), are considered only once. Let $\mathscr{M}$ be the $\mathbf{Z}\left[v^{-1}\right]$ submodule of $U^{+}$generated by $\mathscr{F}$. We have a bijection $\sigma: \mathscr{F} \cong B_{\mathbf{i}}$; to an element (e) we associate $E_{\mathbf{i}}^{m, m^{\prime \prime}, m^{\prime}-m^{\prime \prime}}$ and to an element (f) we associate $E_{\mathbf{i}}^{m^{\prime}-m, m, m^{\prime \prime}}$. The previous computations show that each element in $\mathscr{F}$ is equal to the corresponding element of form $E_{\mathbf{i}}^{a, b, c}$ plus a linear combination with coefficients in $v^{-1} \mathbf{Z}\left[v^{-1}\right]$ of elements of the form $E_{\mathbf{i}}^{a^{\prime}, b^{\prime}, c^{\prime}}$ with $b^{\prime}<b$, $a+b=a^{\prime}+b^{\prime}, b+c=b^{\prime}+c^{\prime}$.

Hence $\mathscr{M}=\mathscr{L}_{\mathbf{i}}, \mathscr{F}$ is a $\mathbf{Z}\left[v^{-1}\right]$-basis of $\mathscr{M}$ and the image of $\mathscr{F}$ under the canonical map $\mathscr{M} \rightarrow \mathscr{M} / v^{-1} \mathscr{M}$ coincides with the image of $B_{\mathrm{i}}$. The automorphism $E_{1} \rightarrow E_{2}, E_{2} \rightarrow E_{1}$ of $\mathbf{U}^{+}$leaves stable $\mathscr{F}, \mathscr{M}$, and takes $\mathscr{L}_{\mathbf{i}}$ onto $\mathscr{L}_{\mathbf{i}^{\prime}}$ and $E_{\mathrm{i}}^{a, b, c}$ to $E_{\mathrm{i}^{\prime}}^{a, b, c}$. Hence we have necessarily $\mathscr{L}_{\mathbf{i}}=\mathscr{L}_{\mathrm{i}^{\prime}}=\mathscr{M}$ and the images of $B_{\mathbf{i}}$ and $B_{\mathbf{i}^{\prime}}$ under $\mathscr{M} \rightarrow \mathscr{M} / v^{-1} \mathscr{M}$ coincide. We obtain a bijection between $B_{\mathbf{i}}$ and $B_{\mathbf{i}^{\prime}}$ as follows: $E_{\mathbf{i}}^{a, b, c}$ corresponds to $E_{\mathbf{i}^{\prime}}^{a^{\prime}, b^{\prime}, c^{\prime}}$ precisely when they have the same image under $\mathscr{M} \rightarrow \mathscr{M} / v^{-1} \mathscr{M}$. This image is then the same as that of an element $\psi$ as in (e) or (f). Assume that $\psi$ is 
as in (e). Then, as we have seen, we have $(a, b, c)=\left(m, m^{\prime \prime}, m^{\prime}-m^{\prime \prime}\right)$ and similarly $\left(a^{\prime}, b^{\prime}, c^{\prime}\right)=\left(m^{\prime}-m, m, m^{\prime \prime}\right)$. Hence

$$
\left(a^{\prime}, b^{\prime}, c^{\prime}\right)=(b+c-\min (a, c), \min (a, c), a+b-\min (a, c)) .
$$

Assume next that $\psi$ is as in (f). Then, as we have seen, we have $(a, b, c)=$ $\left(m^{\prime}-m, m, m^{\prime \prime}\right)$ and similarly $\left(a^{\prime}, b^{\prime}, c^{\prime}\right)=\left(m, m^{\prime \prime}, m^{\prime}-m^{\prime \prime}\right)$. Hence $(\mathrm{g})$ holds again. This completes the proof of the proposition.

2.4. We now return to the setup in 2.1. Let $\widehat{\mathscr{X}}$ be the set of all morphisms of graphs $\sigma: \mathbf{X} \rightarrow \widetilde{\mathbf{X}}$ such that $p r_{1} \circ \sigma=1$. We may regard $\widehat{\mathscr{X}}$ as the set of all maps $\phi: \mathscr{X} \rightarrow \mathbf{N}^{\nu}$ such that $R_{\mathbf{i}}^{\mathbf{i}^{\prime}}(\phi(\mathbf{i}))=\phi\left(\mathbf{i}^{\prime}\right)$ whenever $\left(\mathbf{i}, \mathbf{i}^{\prime}\right)$ are joined in $\mathbf{X}$.

We define a map

$$
B \rightarrow \widehat{\mathscr{X}}
$$

as follows. To $\psi \in B$ we associate the function $\phi: \mathscr{X} \rightarrow \mathbf{N}^{\nu}$ given by $\phi(\mathbf{i})=\mathbf{c}$ where $\pi\left(E_{\mathbf{i}}^{\mathbf{c}}\right)=\psi$. From 2.3 it follows immediately that $\phi \in \widehat{\mathscr{X}}$.

For any $\mathbf{i} \in \mathscr{X}$, we have a map $\widehat{\mathscr{X}} \cong \mathbf{N}^{\nu}$ defined by $\phi \rightarrow \phi(\mathbf{i})$ and a bijection

$$
\mathbf{N}^{\nu} \rightarrow B
$$

defined by $\mathbf{c} \rightarrow \pi\left(E_{\mathbf{i}}^{\mathbf{c}}\right) \in B$. Their composition is a map $\widehat{\mathscr{X}} \rightarrow B$. It is clear that this is the inverse of the map (a). Thus we have

Corollary 2.5. The map 2.4(a) is a bijection.

2.6. Let $\mathbf{i}, \mathbf{i}^{\prime}$ be two elements of $\mathscr{X}$. We define a bijection $R_{\mathrm{i}}^{\mathrm{i}^{\prime}}: N^{\nu} \cong N^{\nu}$ as the composition

$$
R_{\mathbf{i}_{p-1}}^{\mathbf{i}_{p}} R_{\mathbf{i}_{p-2}}^{\mathbf{i}_{p-1}} \cdots R_{\mathbf{i}_{1}}^{\mathbf{i}_{2}}
$$

where $\mathbf{i}=\mathbf{i}_{1}, \mathbf{i}_{2}, \ldots, \mathbf{i}_{p}=\mathbf{i}^{\prime}$ is a sequence of vertices such that any two consecutive terms of this sequence are joined in $\mathbf{X}$. (We use the connectedness of this graph.) This bijection is independent of the choice of sequence: it is equal to the composition of the bijection 2.4 (b) (corresponding to $i$ ) with the inverse of the bijection $2.4(\mathrm{~b})$ (corresponding to $\mathrm{i}^{\prime}$ ).

2.7. For each $\mathbf{d}=\left(d_{1}, \ldots, d_{n}\right) \in \mathbf{N}^{n}$ we denote by $\mathbf{U}_{\mathbf{d}}^{+}$the set of all elements $\xi \in \mathbf{U}^{+}$such that $K_{i} \xi=v^{d_{i}} \xi K_{i}$ for all $i$. The elements in $\mathbf{U}_{\mathbf{d}}^{+}$are said to be homogeneous (of homogeneity $\mathbf{d}$ ). The subspaces $\mathbf{U}_{\mathbf{d}}^{+}$(for various $\mathbf{d}$ ) form a direct sum decomposition of $\mathbf{U}^{+}$; moreover, their intersections with $U^{+}$form a direct sum decomposition of $U^{+}$.

2.8. Let $\mathbf{i} \in \mathscr{X}$. We associate to $\mathbf{i}$ the sequence

$$
\alpha^{1}, \alpha^{2}, \ldots, \alpha^{\nu}
$$


defined by $\alpha^{k}=s_{i_{1}} s_{i_{2}} \cdots s_{i_{k-1}}\left(\alpha_{i_{k}}\right)$ for $k=1,2, \ldots, \nu$. This sequence contains each root in $R^{+}$exactly once. We can write uniquely

$$
\alpha^{k}=\sum_{j=1}^{n} p_{j}^{k} \alpha_{j}, \quad 1 \leq k \leq \nu
$$

where $p_{j}^{k} \in \mathbf{N}$.

We now define a map $\chi_{\mathbf{i}}: \mathbf{N}^{\nu} \rightarrow \mathbf{N}^{n}$ by

$$
\mathbf{c}=\left(c_{1}, c_{2}, \ldots, c_{\nu}\right) \rightarrow \mathbf{d}=\left(d_{1}, \ldots, d_{n}\right) \quad \text { where } d_{j}=\sum_{k=1}^{\nu} p_{j}^{k} c_{k} .
$$

The fibres of this map are finite. An element of $\mathbf{N}^{\nu}$ in the fibre of $\chi_{\mathbf{i}}$ over $\mathbf{d}$ is said to have i-homogeneity d.

2.9. If $\mathbf{i}, \mathbf{i}^{\prime}$ are joined in the graph $\mathbf{X}$, one can check easily that $\chi_{\mathbf{i}}=\chi_{\mathbf{i}^{\prime}} R_{\mathbf{i}}^{\mathbf{i}^{\prime}}$ : $\mathbf{N}^{\nu} \rightarrow \mathbf{N}^{n}$. Hence there is a unique map $\chi: \hat{\mathscr{Z}} \rightarrow \mathbf{N}^{n}$ such that $\chi(\phi)=\chi_{\mathbf{i}}(\phi(\mathbf{i}))$ for any $\mathbf{i} \in \mathscr{X}$ and any $\phi \in \hat{\mathscr{X}}$. This map, composed with the map 2.4(a) gives a map

$$
B \rightarrow \mathbf{N}^{n} \text {. }
$$

2.10. If $\mathbf{i} \in \mathscr{Z}$, the basis $B_{\mathbf{i}}$ consists of homogeneous elements. The part of this basis that is contained in $\mathrm{U}_{\mathrm{d}}^{+}$corresponds under the bijection $\pi: B_{\mathrm{i}} \cong B$ to the inverse image of $\mathbf{d}$ under the map 2.9(a).

2.11. Let $\mathbf{i}=\left(i_{1}, \ldots, i_{\nu}\right) \in \mathscr{X}$ and let $\mathbf{i}^{\prime}=\left(i_{1}^{\prime}, \ldots, i_{\nu}^{\prime}\right)$ be defined by $s_{i_{j}}^{\prime}=$ $w_{0} s_{i_{\nu-j+1}} w_{0}^{-1}$. Then $\mathbf{i}^{\prime} \in \mathscr{X}$. One verifies easily that for any $k \in[1, \nu]$ we have

$$
s_{i_{k-1}} s_{i_{k-2}} \cdots s_{i_{1}} s_{i_{1}^{\prime}} s_{i_{2}^{\prime}} \cdots s_{i_{\nu-k}^{\prime}} s_{i_{\nu-k+1}^{\prime}}=w_{0}
$$

and

$$
s_{i_{k-1}} s_{i_{k-2}} \cdots s_{i_{1}} s_{i_{1}^{\prime}} s_{i_{2}^{\prime}} \cdots s_{i_{\nu-k}^{\prime}}\left(\alpha_{i_{\nu-k+1}^{\prime}}\right)=\alpha_{i_{k}}
$$

Hence, using 1.3(c), we have

$$
\tilde{T}_{i_{k-1}} \tilde{T}_{i_{k-2}} \ldots \tilde{T}_{i_{1}} \tilde{T}_{i_{1}^{\prime}} \tilde{T}_{i_{2}^{\prime}} \ldots \widetilde{T}_{i_{\nu-k}^{\prime}}\left(E_{i_{\nu-k+1}^{\prime}}\right)=E_{i_{k}}
$$

It follows that

$$
\tilde{T}_{i_{1}^{\prime}} \widetilde{T}_{i_{2}^{\prime}} \cdots \widetilde{T}_{i_{\nu-k}^{\prime}}\left(E_{i_{\nu-k+1}^{\prime}}\right)=\tilde{T}_{i_{1}}^{-1} \tilde{T}_{i_{2}}^{-1} \cdots \tilde{T}_{i_{k-1}}^{-1}\left(E_{i_{k}}\right)=\Psi\left(\tilde{T}_{i_{1}} \tilde{T}_{i_{2}} \cdots \tilde{T}_{i_{k-1}}\left(E_{i_{k}}\right)\right) .
$$

This identity shows that

$$
\Psi\left(E_{\mathbf{i}}^{\mathfrak{c}}\right)=E_{\mathbf{i}^{\prime}}^{\mathbf{c}^{\prime}}
$$

where $\mathbf{c}^{\prime}=\left(c_{1}^{\prime}, \ldots, c_{\nu}^{\prime}\right)$ is related to $\mathbf{c}=\left(c_{1}, \ldots, c_{\nu}\right)$ by $c_{j}^{\prime}=c_{\nu-j+1}$. In particular, we have

$$
\Psi\left(B_{\mathbf{i}}\right)=B_{\mathbf{i}^{\prime}}
$$


and

$$
\Psi(\mathscr{L})=\mathscr{L}
$$

\section{Definition of the canonical basis B}

3.1. Applying ${ }^{-}: U^{+} \rightarrow U^{+}$to $\mathscr{L}$ we obtain a $\mathrm{Z}[v]$-submodule $\overline{\mathscr{L}}$ of $U^{+}$.

Theorem 3.2. (a) The restriction of $\pi: \mathscr{L} \rightarrow \mathscr{L} / v^{-1} \mathscr{L}$ defines an isomorphism of Z-modules $\pi^{\prime}: \mathscr{L} \cap \overline{\mathscr{L}} \cong \mathscr{L} / v^{-1} \mathscr{L}$. Hence, if we set $\mathbf{B}=\pi^{\prime-1}(B)$, then $\mathbf{B}$ is a $\mathbf{Z}$-basis of $\mathscr{L} \cap \overline{\mathscr{L}}$.

(b) $\mathbf{B}$ is a $\mathbf{Z}\left[v^{-1}\right]$-basis of $\mathscr{L}$, a $\mathbf{Z}[v]$-basis of $\overline{\mathscr{L}}$, an A-basis of $U^{+}$and an $A^{\prime}$-basis of $\mathbf{U}^{+}$.

(c) Each element of $\mathbf{B}$ is fixed by ${ }^{-}: U^{+} \rightarrow U^{+}$.

The proof will be given in 7.10, 7.11. We call $\mathbf{B}$ the canonical basis.

Proposition 3.3. $\Psi(\mathbf{B})=\mathbf{B}$.

By $2.11\left(\right.$ b), $\Psi$ maps $\mathscr{L}$ onto itself; it induces an automorphism of $\mathscr{L} / v^{-1} \mathscr{L}$ that maps $B$ onto itself, as we see from $2.11(\mathrm{a})$. Clearly, $\Psi$ commutes with - : $\mathbf{U} \rightarrow \mathbf{U}$ and with $\pi: \mathscr{L} \rightarrow \mathscr{L} / v^{-1} \mathscr{L}$; hence it leaves stable $\mathscr{L} \cap \overline{\mathscr{L}}$ and also B. The proposition follows.

3.4. Examples. In type $A_{1}$, the basis $\mathbf{B}$ consists of the elements $E_{1}^{(m)}, m \in \mathbf{N}$. In type $A_{2}$, the basis $\mathbf{B}$ consists of the elements

$$
\begin{gathered}
E_{1}^{(m)} E_{2}^{\left(m^{\prime}\right)} E_{1}^{\left(m^{\prime \prime}\right)}, \\
E_{2}^{(m)} E_{1}^{\left(m^{\prime}\right)} E_{2}^{\left(m^{\prime \prime}\right)}
\end{gathered}
$$

for various $\left(m, m^{\prime}, m^{\prime \prime}\right) \in \mathbf{N}^{3}$ such that $m^{\prime} \geq m+m^{\prime \prime}$ where the elements

$$
E_{1}^{(m)} E_{2}^{\left(m+m^{\prime \prime}\right)} E_{1}^{\left(m^{\prime \prime}\right)}=E_{2}^{\left(m^{\prime \prime}\right)} E_{1}^{\left(m+m^{\prime \prime}\right)} E_{2}^{(m)},
$$

which are both of type (a) and (b), are considered only once. This follows from the arguments in the proof of 2.3. In general, the basis $\mathbf{B}$ may contain nonmonomials; for example, in type $A_{3}$ (with generators $E_{1}, E_{2}, E_{3}$, such that $E_{1}, E_{3}$ commute), it contains the element

$$
E_{2}^{(2)} E_{1} E_{3} E_{2}-E_{2}^{(3)} E_{1} E_{3}=E_{2} E_{1} E_{3} E_{2}^{(2)}-E_{1} E_{3} E_{2}^{(3)}
$$

\section{QUIVERS}

4.1. The connection between the representation theory of quivers [G, BGP] and that of quantized enveloping algebra has been first pointed out by Ringel [R]. We shall recall some basic facts on quivers; we shall follow the methods of [BGP] except for the fact that we shall use in an essential way the reduced expressions for $w_{0}$. Recall that the Dynkin graph of $\left(a_{i j}\right)$ has $[1, n]$ as set of vertices; 
$i, j$ form an edge precisely when $a_{i j}=-1$. Assume given an orientation $\Omega$ of this Dynkin graph, i.e., a collection of arrows $i \rightarrow j$, one for each edge $i, j$ in the Dynkin graph. Let $F$ be a fixed field. Let $M \Omega$ be the category of "modules" of this oriented graph, or quiver. An object of $M \Omega$ is a collection of finite-dimensional $F$-vector spaces $V_{i} \quad(i \in[1, n])$ and of $F$-linear maps $f_{i j}: V_{i} \rightarrow V_{j}$ defined for $i \rightarrow j$. A morphism from this object to another object $\left(V_{i}^{\prime}, f_{i j}^{\prime}\right)$ is a collection of $F$-linear maps $g_{i}: V_{i} \rightarrow V_{i}^{\prime} \quad(i \in[1, n])$, such that $f_{i j}^{\prime} g_{i}=g_{j} f_{i j}$ for all $i \rightarrow j$. Then $M \Omega$ is an abelian category in an obvious way.

4.2. The dimension of $\mathbf{V}=\left(V_{i}, f_{i j}\right)$ is the sequence

$$
\operatorname{dim}(\mathbf{V})=\left(\operatorname{dim}\left(V_{1}\right), \ldots, \operatorname{dim}\left(V_{n}\right)\right) \in \mathbf{N}^{n} .
$$

For $i \in[1, n]$ let $e_{i}$ be the module defined by $V_{i}=F, V_{j}=0$ for $j \neq 0$. This module is simple and any simple module is isomorphic to an $e_{i}$ for a unique $i$.

4.3. Assume that $i \in[1, n]$ is a sink (resp. a source) of $\Omega$, i.e., that there is no arrow $i \rightarrow j$ (resp. $j \rightarrow i$ ) in $\Omega$. Let $M_{i}^{+} \Omega$ (resp. $M_{i}^{-} \Omega$ ) denote the full subcategory of $M \Omega$ whose objects are the $\left(V_{i}, f_{i j}\right)$ such that $\bigoplus_{j} f_{j i}: \bigoplus_{j} V_{j} \rightarrow$ $V_{i}$ is surjective (resp. $\bigoplus_{j} f_{i j}: V_{i} \rightarrow \bigoplus_{j} V_{j}$ is injective); in both cases, $j$ runs over the vertices of the Dynkin graph that are joined with $i$. The following statement is easily verified.

(a) Let $\mathbf{V}$ be a module in $M \Omega$. We have $\mathbf{V} \in M_{i}^{+} \Omega$ (resp. $\mathbf{V} \in M_{i}^{-} \Omega$ ) if and only if $\operatorname{Hom}_{M \Omega}\left(\mathbf{V}, e_{i}\right)=0$ (resp. $\operatorname{Hom}_{M \Omega}\left(e_{i}, \mathbf{V}\right)=0$ ).

Let $s_{i} \Omega$ be the orientation obtained from $\Omega$ by reversing each arrow that ends (resp. starts) at $i$; then $i$ is a source (resp. a sink) of $s_{i} \Omega$.

Following [BGP] we define the "reflection functor" $\Phi_{i}^{+}: M \Omega \rightarrow M_{i}^{-} s_{i} \Omega$ (resp. $\Phi_{i}^{-}: M \Omega \rightarrow M_{i}^{+} s_{i} \Omega$ ) by associating to an object $\left(V_{h}, f_{h h^{\prime}}\right) \in M \Omega$ the object $\left(V_{k}^{\prime}, f_{k k^{\prime}}^{\prime}\right) \in M s_{i} \Omega$ defined by $V_{i}^{\prime}=\operatorname{Ker}\left(\bigoplus_{j} f_{j i}: \bigoplus_{j} V_{j} \rightarrow V_{i}\right), \quad V_{h}^{\prime}=$ $V_{h}$ for $h \neq i$ (resp. $V_{i}^{\prime}=\operatorname{Coker}\left(\bigoplus_{j} f_{i j}: V_{i} \rightarrow \bigoplus_{j} V_{j}\right), \quad V_{h}^{\prime}=V_{h}$ for $\left.h \neq i\right), j$ as above; the maps $f_{k k^{\prime}}^{\prime}$ are the obvious ones. On morphisms, $\boldsymbol{\Phi}_{i}^{+}$(resp. $\boldsymbol{\Phi}_{i}^{-}$) is defined in the obvious way.

4.4. Assume that $i$ is a sink of $\Omega$. For any module $\mathbf{V} \in M \Omega$ there is a canonical exact sequence

$$
0 \longrightarrow \boldsymbol{\Phi}_{i}^{-} \boldsymbol{\Phi}_{i}^{+}(\mathbf{V}) \longrightarrow \mathbf{V} \longrightarrow \mathbf{V}(i) \longrightarrow 0
$$

where $\mathbf{V}(i)$ has $i$-component $\operatorname{Coker}\left(\bigoplus_{j} f_{j i}: \bigoplus_{j} V_{j} \rightarrow V_{i}\right)$ and its other components are 0 ( $j$ runs over the neighbors of $i$ in the Dynkin graph). In particular,

$$
\Phi_{i}^{-} \Phi_{i}^{+}(\mathbf{V}) \cong \mathbf{V} \quad \text { if and only if } \mathbf{V} \in M_{i}^{+} \Omega
$$

and the restriction of $\Phi_{i}^{+}$defines an equivalence of categories

$$
M_{i}^{+} \Omega \cong M_{i}^{-} s_{i} \Omega
$$


whose inverse is given by the restriction of $\Phi_{i}^{-}$. If $\mathbf{V} \in M_{i}^{+} \Omega$ corresponds to $\mathbf{V}^{\prime} \in M_{i}^{-} s_{i} \Omega$ under $(\mathrm{c})$ then

$$
\operatorname{dim}\left(\mathbf{V}^{\prime}\right)=\left(d_{1}^{\prime}, \ldots, d_{n}^{\prime}\right) \text { and } \operatorname{dim}(\mathbf{V})=\left(d_{1}, \ldots, d_{n}\right)
$$

are related by

$$
d_{1}^{\prime} \alpha_{1}+\cdots+d_{n}^{\prime} \alpha_{n}=s_{i}\left(d_{1} \alpha_{1}+\cdots+d_{n} \alpha_{n}\right)
$$

(notation of 1.2).

4.5. Let $\mathbf{M}, \mathbf{M}^{\prime}, \mathbf{M}^{\prime \prime}$ be modules in $M \Omega$; assume that the following conditions are satisfied:

(a) $\mathbf{M}$ is isomorphic to $\mathbf{M}^{\prime} \oplus \mathbf{M}^{\prime \prime}$,

(b) any exact sequence $0 \longrightarrow \mathbf{M}^{\prime \prime} \longrightarrow \widetilde{\mathbf{M}} \longrightarrow \mathbf{M}^{\prime} \longrightarrow 0$ in $M \Omega$ is split,

(c) there is a unique submodule of $\mathbf{M}$ that is isomorphic to $\mathbf{M}^{\prime \prime}$ and is such that the quotient of $\mathbf{M}$ by it is isomorphic to $\mathbf{M}^{\prime}$.

We express this by the notation $\mathbf{M} \cong \mathbf{M}^{\prime} \star \mathbf{M}^{\prime \prime}$. For example, in 4.4(a), we have

$$
\mathbf{V} \cong \mathbf{V}(i) \star \Phi_{i}^{-} \Phi_{i}^{+}(\mathbf{V})
$$

Assume that $i$ is a sink of $\Omega$, and that $\mathbf{M}^{\prime}, \mathbf{M}^{\prime \prime} \in M_{i}^{+} \Omega$; let $\overline{\mathbf{M}^{\prime}}, \overline{\mathbf{M}^{\prime \prime}}$ be the corresponding objects in $M_{i}^{-} s_{i} \Omega$ (under 4.4(c)). Assume also that $\overline{\mathbf{M}} \in M s_{i} \Omega$ satisfies $\overline{\mathbf{M}} \cong \overline{\mathbf{M}^{\prime}} \star \overline{\mathbf{M}^{\prime \prime}}$. Then

(e) $\overline{\mathbf{M}} \in M_{i}^{-} s_{i} \Omega$ and the corresponding object (under 4.4(c)) $\mathbf{M} \in M_{i}^{+} \Omega$ satisfies $\mathbf{M} \cong \mathbf{M}^{\prime} \star \mathbf{M}^{\prime \prime}$.

The proof is standard; it is left to the reader.

4.6. Assume that $i$ is a sink of $\Omega$ and that $\mathbf{V}$ is an indecomposable module in $M \Omega$, not isomorphic to $e_{i}$. From 4.5(d) it follows that $\mathbf{V}$ is in $M_{i}^{+} \Omega$. It also follows that 4.4(c) defines a bijection between the following two sets:

(a) the set of indecomposable modules (up to isomorphism) other than $e_{i}$ in $M_{i}^{+} \Omega$ and

(b) the set of indecomposable modules (up to isomorphism) other than $e_{i}$ in $M_{i}^{-} s_{i} \Omega$.

(Note that $e_{i}$ is defined for both $\Omega$ and $s_{i} \Omega$.) On the other hand, $\Phi_{i}^{+}\left(e_{i}\right)=0$ $\left(e_{i} \in M \Omega\right)$.

4.7. Assume that we are given an element $\mathbf{i}=\left(i_{1}, i_{2}, \ldots, i_{\nu}\right) \in \mathscr{Z}$. For $1 \leq k \leq \nu+1$ we set $\left.\Omega_{k}=s_{i_{k-1}}\left(\ldots\left(s_{i_{2}}\left(s_{i_{1}} \Omega\right) \ldots\right)\right)\right)$. We assume that $i_{k}$ is a sink of $\Omega_{k}$ for $1 \leq k \leq \nu$. (We then say that $\mathbf{i}$ is adapted to $\Omega$.) If $\mathbf{V}$ is a module in $M \Omega, k \in[0, \nu]$, and $k^{\prime} \in[1, k+1]$ we set

$$
\begin{gathered}
\mathbf{V}^{k}=\Phi_{i_{k}}^{+} \ldots \Phi_{i_{2}}^{+} \Phi_{i_{1}}^{+}(\mathbf{V}) \in M \Omega_{k+1}, \\
\mathbf{V}^{k^{\prime}, k}=\Phi_{i_{k^{\prime}}}^{-} \cdots \Phi_{i_{k-1}}^{-} \Phi_{i_{k}}^{-}\left(\mathbf{V}^{k}\right) \in M \Omega_{k^{\prime}}
\end{gathered}
$$


In particular,

$$
\mathbf{V}^{k+1, k}=\mathbf{V}^{k}
$$

We have

$$
\mathbf{V}^{\nu}=0
$$

Indeed, assume that $\mathbf{V}^{\nu}$ is nonzero for some $\mathbf{V}$. We may assume that $\mathbf{V}$ is indecomposable. From 4.6 it follows that $\mathbf{V} \in M_{i_{1}}^{+} \Omega_{1}, \Phi_{i_{1}}^{+} \mathbf{V} \in M_{i_{2}}^{+} \Omega_{2}, \Phi_{i_{2}}^{+} \Phi_{i_{1}}^{+} \mathbf{V} \in$ $M_{i_{3}}^{+} \Omega_{3}$, etc. Now using $4.4(\mathrm{~d})$ we see that $\operatorname{dim}\left(\mathbf{V}^{\nu}\right)=\left(d_{1}^{\prime}, \ldots, d_{n}^{\prime}\right)$ is related to $\operatorname{dim}(\mathbf{V})=\left(d_{1}, \ldots, d_{n}\right)$ by $\left(d_{1}^{\prime} \alpha_{1}+\cdots, d_{n}^{\prime} \alpha_{n}\right)=w_{0}\left(d_{1} \alpha_{1}+\cdots, d_{n} \alpha_{n}\right)$. In the left-hand side of this equality the coefficients are in $\mathbf{N}$ and not all are zero; in the right-hand side the coefficients are in $-\mathbf{N}$; this is a contradiction.

4.8. In the setup of 4.7 , let $X_{k}$ be a module in $M \Omega_{k}$ whose component at any vertex $\neq i_{k}$ is 0 ; assume that $2 \leq k^{\prime} \leq k$. Then

(a) the module $\mathbf{P}^{\prime}=\Phi_{i_{k^{\prime}}}^{-} \cdots \Phi_{i_{k-2}}^{-} \Phi_{i_{k-1}}^{-}\left(X_{k}\right)$ is in $M_{i_{k^{\prime}-1}}^{-} \Omega_{k^{\prime}}$.

Indeed, we can assume that $X_{k}=e_{i_{k}}$. From 4.6 we see that $\mathbf{P}^{\prime}$ is indecomposable. Let $\mathbf{d}=\operatorname{dim} \mathbf{P}^{\prime} \in \mathbf{Z}^{n} \cong Q$. If $\mathbf{P}^{\prime}$ is not in $M_{i_{k^{\prime}-1}}^{-} \Omega_{k^{\prime}}$ then $\mathbf{P}^{\prime} \cong e_{i_{k^{\prime}-1}}($ again by 4.6$)$ and then $s_{i_{k^{\prime}-1}}\left(\operatorname{dim} \mathbf{P}^{\prime}\right) \in \mathbf{Z}^{n} \cong Q$ would have some strictly negative coordinate; on the other hand, this vector is equal to $s_{i_{k^{\prime}-1}} s_{i_{k^{\prime}}} \cdots s_{i_{k-2}} s_{i_{k-1}}\left(\alpha_{i_{k}}\right)$, which has positive coordinates since $s_{i_{k^{\prime}-1}} s_{i_{k^{\prime}}} \cdots$ $s_{i_{k-2}} s_{i_{k-1}} s_{i_{k}}$ is a reduced expression in $W$. This contradiction proves (a).

Now let $0 \longrightarrow \mathbf{M}^{\prime \prime} \longrightarrow \mathbf{M} \longrightarrow \mathbf{M}^{\prime} \longrightarrow 0$ be an exact sequence in $M \Omega_{k}^{\prime}$ such that $\mathbf{M}^{\prime} \in M_{i_{k^{\prime}-1}}^{-} \Omega_{k^{\prime}}$. Then

$$
0 \longrightarrow \Phi_{i_{k^{\prime}-1}}^{-}\left(\mathbf{M}^{\prime \prime}\right) \longrightarrow \Phi_{i_{k^{\prime}-1}}^{-}(\mathbf{M}) \longrightarrow \Phi_{i_{k^{\prime}-1}}^{-}\left(\mathbf{M}^{\prime}\right) \longrightarrow 0
$$

is an exact sequence in $M \Omega_{k^{\prime}-1}$. This follows immediately from the "snake lemma."

Proposition 4.9. Assume that $\mathbf{i} \in \mathscr{X}$ is adapted to $\Omega$. Let $\mathbf{V}$ be an object in $M \Omega$.

(a) We have a canonical filtration (in $M \Omega$ )

$$
\mathbf{V}=\mathbf{V}^{1,0} \supset \mathbf{V}^{1,1} \supset \cdots \supset \mathbf{V}^{1, \nu-1} \supset \mathbf{V}^{1, \nu}=0
$$

We have $\mathbf{V}^{1, k-1} / \mathbf{V}^{1, k}=P_{k}$ where

$$
P_{k}=\Phi_{i_{1}}^{-} \Phi_{i_{2}}^{-} \cdots \Phi_{i_{k-1}}^{-}\left(X_{k}\right)
$$

and $X_{k}=\mathbf{V}^{k-1} / \mathbf{V}^{k, k}$ is a module in $M \Omega_{k}$ whose $j$-component is zero for any $j \neq i_{k}$. (Notation of 4.7.)

(b) We have

$$
\mathbf{V}^{1, k-1} \cong P_{k} \star \mathbf{V}^{1, k}
$$


for any $k \in[1, \nu]$. Hence,

$$
\mathbf{V}=P_{1} \star\left(P_{2} \star\left(\cdots \star P_{\nu}\right) \ldots\right)
$$

(c) If $k^{\prime}<k$, we have

$$
\operatorname{Hom}_{M \Omega}\left(P_{k}, P_{k^{\prime}}\right)=0 \text {. }
$$

The proof will be given in 4.10, 4.11.

4.10. From 4.4(a) we have (for $k \in[1, \nu]$ ) a canonical exact sequence in $M \Omega_{k}$

$$
0 \longrightarrow \mathbf{V}^{k, k} \longrightarrow \mathbf{V}^{k-1} \longrightarrow X_{k} \longrightarrow 0
$$

where $X_{k}$ is concentrated at the vertex $i_{k}$. Applying 4.8(a) and (b) repeatedly, we deduce that for any $k^{\prime} \in[1, k]$ we have a canonical exact sequence

$$
0 \longrightarrow \mathbf{V}^{k^{\prime}, k} \longrightarrow \mathbf{V}^{k^{\prime}, k-1} \longrightarrow \Phi_{i_{k^{\prime}}}^{-} \Phi_{i_{k^{\prime}+1}^{-}}^{-} \cdots \Phi_{i_{k-1}}^{-} X_{k} \longrightarrow 0 \text {. }
$$

4.11. We will show that

$$
\mathbf{V}^{k^{\prime}, k-1} \cong \Phi_{i_{k^{\prime}}}^{-} \Phi_{i_{k^{\prime}+1}^{-}}^{-} \cdots \Phi_{i_{k-1}}^{-}\left(X_{k}\right) \star \mathbf{V}^{k^{\prime}, k}
$$

for any $k \in[1, \nu], k^{\prime} \in[1, k]$. For $k^{\prime}=k$ this follows from 4.5(d). Assume that (a) is known for some $k^{\prime} \leq k, k^{\prime} \geq 2$; we shall deduce from this the analogous statement for $k^{\prime}-1, k$ by applying to it $\Phi_{i_{k^{\prime}-1}^{-}}^{-}$. In view of $4.5(\mathrm{e})$, it is enough to verify that

$$
\mathbf{V}^{k^{\prime}, k} \in M_{i_{k^{\prime}-1}^{-}}^{-} \Omega_{k^{\prime}}
$$

and

$$
\Phi_{i_{k^{\prime}}}^{-} \Phi_{i_{k^{\prime}+1}}^{-} \ldots \Phi_{i_{k-1}}^{-}\left(X_{k}\right) \in M_{i_{k^{\prime}-1}}^{-} \Omega_{k^{\prime}}
$$

The last inclusion follows from 4.8(a). Applying 4.10(b) repeatedly, we see that $\mathbf{V}^{k^{\prime}, k}$ is a submodule of $\mathbf{V}^{k^{\prime}, k^{\prime}-1}=\mathbf{V}^{k^{\prime}-1}$, which, by definition, is in $M_{i_{k^{\prime}-1}}^{-} \Omega_{k^{\prime}}$; hence so is any submodule of it. Thus, (a) is proved. We now take $k^{\prime}=1$ in (a) and we see that $4.9(\mathrm{a})$, (b) hold.

We now prove $4.9(\mathrm{c})$. Assume that $\operatorname{Hom}_{M \Omega}\left(P_{k}, P_{k^{\prime}}\right) \neq 0$ for some $k^{\prime}<k$. Applying successively the functors $\Phi_{i_{1}}^{+}, \quad \Phi_{i_{2}}^{+}, \ldots, \Phi_{i_{k^{\prime}-1}^{+}}^{+}$and using 4.4(c), we deduce that there exists a nonzero element $\xi \in \operatorname{Hom}\left(\mathbf{P}, X_{k^{\prime}}\right)$ where

$$
\mathbf{P}=\Phi_{i_{k^{\prime}}}^{-} \Phi_{i_{k^{\prime}+1}}^{-} \ldots \Phi_{i_{k-1}}^{-}\left(X_{k}\right) \in M \Omega_{k^{\prime}}
$$

This contradicts $4.3(\mathrm{a})$ since $\mathbf{P} \in M_{i_{k^{\prime}}}^{+} \Omega_{k^{\prime}}$. This contradiction proves 4.9(c).

Proposition 4.12. We fix an orientation $\Omega$. The following hold.

(a) For any $\alpha \in R^{+}$there is a unique indecomposable module (up to isomorphism) denoted $e_{\alpha} \in M \Omega$ such that $\operatorname{dim}\left(e_{\alpha}\right)=\left(d_{1}, \ldots, d_{n}\right), \alpha=d_{1} \alpha_{1}+\cdots+$ $d_{n} \alpha_{n}$; any indecomposable module is isomorphic to $e_{\text {cx }}$ for a unique $\alpha$ (Gabriel's theorem). In particular, we have $e_{\alpha_{i}}=e_{i}$. 
(b) There exists some $\mathbf{i} \in \mathscr{Z}$ adapted to $\Omega$.

(c) There exists a total order $\alpha^{1}, \alpha^{2}, \ldots, \alpha^{\nu}$ on $R^{+}$such that

$$
\operatorname{Hom}_{M \Omega}\left(e_{\alpha^{k}}, e_{\alpha^{k^{\prime}}}\right)=0
$$

for any $k^{\prime}<k$.

We first show that (b) implies (a). Let $i$ be adapted to $\Omega$. From 4.6 we see that for $k \in[1, \nu], \Phi_{i_{1}}^{-} \Phi_{i_{2}}^{-} \cdots \Phi_{i_{k-1}}^{-}\left(e_{i_{k}}\right)$ is an indecomposable module in $M \Omega$ of dimension $\left(d_{1}, \ldots, d_{n}\right)$ where

$$
s_{i_{1}} s_{i_{2}} \cdots s_{i_{k-1}}\left(\alpha_{i_{k}}\right)=d_{1} \alpha_{1}+\cdots+d_{n} \alpha_{n} .
$$

From 4.9(b) we see that any indecomposable module in $M \Omega$ is of the form above. Since any $\alpha \in R^{+}$is of the form (d) for a unique $k \in[1, \nu]$, we see that (a) holds.

Note that (b) implies (c) by 4.9(c).

We now show that (a) and (c) imply (b). Consider a total order as in (c). It is clear from the definition that $e_{\alpha^{1}}$ is necessarily a simple module (hence $\alpha^{1}=\alpha_{i_{1}}$ for some $\left.i_{1} \in[1, n]\right)$ and that $i_{1}$ is necessarily a sink of $\Omega$. The sequence $s_{i_{1}}\left(\alpha^{2}\right), s_{i_{1}}\left(\alpha^{3}\right), \ldots s_{i_{1}}\left(\alpha^{\nu}\right), \alpha^{1}$ plays the same role for $s_{i_{1}} \Omega$ as $\alpha^{1}, \ldots, \alpha^{\nu}$ for $\Omega$; the indecomposable modules in $M s_{i_{1}} \Omega$ corresponding to the terms of this sequence are $\Phi_{i_{1}}^{+} e_{\alpha^{2}}, \ldots, \Phi_{i_{1}}^{+} e_{\alpha^{\nu}}, e_{\alpha_{i_{1}}}$. (We use 4.6 and 4.3(a).) By the previous argument, we see that $s_{i_{1}}\left(\alpha^{2}\right)=\alpha_{i_{2}}$ where $i_{2} \in[1, n]$ is a sink of $s_{i_{1}} \Omega$. Continuing in this way, we find a sequence $\left(i_{1}, i_{2}, \ldots, i_{\nu}\right)$ in $[1, n]$ such that $i_{k}$ is a sink of $s_{i_{1}} s_{i_{2}} \cdots s_{i_{k-1}} \Omega$ for $1 \leq k \leq \nu$ and $s_{i_{1}} s_{i_{2}} \cdots s_{i_{k-1}}\left(\alpha_{i_{k}}\right)=\alpha^{k}$ for $1 \leq k \leq \nu$. The last condition implies that $s_{i_{1}} s_{i_{2}} \cdots s_{i_{\nu}}$ is a reduced expression for $w_{0}$. Hence $\left(i_{1}, i_{2}, \ldots, i_{\nu}\right)$ is adapted to $\Omega$ and (b) holds.

Next we assume that our (oriented) Dynkin graph is embedded as a subgraph of a larger (oriented) Dynkin graph and that the proposition is already known for the larger graph. Clearly, the indecomposable modules for the smaller graph may be identified with the indecomposable modules for the larger graph, which are zero at vertices outside the smaller graph. Hence (a) for the larger graph implies (a) for the smaller graph. Similarly, (c) for the larger graph implies (c) for the smaller graph: we consider an order as in (c) for the larger graph and we discard the roots that do not belong to the smaller graph. We find an order as in (c) for the smaller graph. By an earlier part of the argument, (b) for the smaller graph follows from (a) and (c) for the same graph. We can choose the imbedding above so that the larger graph has the following property: the longest element in the Weyl group is central. Thus, we have reduced the general case to the case where $w_{0}$ is central in $W$. In this case, we can define a Coxeter element $c \in W$ (as in [BGP]) by $c=s_{i_{1}} s_{i_{2}} \cdots s_{i_{n}}$ where $i_{1}, \ldots, i_{n}$ is a permutation of $1, \ldots, n$ such that $i_{j} \rightarrow i_{j^{\prime}}$ in $\Omega$ implies $j^{\prime}<j$. Then $c$ has even order $h$ and $c^{h / 2}=w_{0}$. For $1 \leq k \leq \nu=n h / 2$ we set $i_{k}=i_{j}$ where 
$k \equiv j(\bmod n), 1 \leq j \leq n$. It is easy to check that $\left(i_{1}, \ldots, i_{\nu}\right)$ is adapted to $M \Omega$. Hence (b) holds; as we have seen earlier, this implies that (a) and (c) hold also. This completes the proof.

4.13. The previous proof shows that there is a 1-1 correspondence between the sequences $\mathrm{i} \in \mathscr{Z}$ that are adapted to $\Omega$ and the total orders on $R^{+}$as in 4.12(c). This is obtained by attaching to $i$ the ordering

$$
\alpha^{1}, \alpha^{2}, \ldots, \alpha^{\nu}
$$

of $R^{+}$given by $s_{i_{1}} s_{i_{2}} \cdots s_{i_{k-1}}\left(\alpha_{i_{k}}\right)=\alpha^{k}$.

One can show that a sequence $\mathbf{i} \in \mathscr{X}$ can be adapted to at most one orientation. (This follows from 4.14(b).)

4.14. Assume $\mathbf{i}=\left(i_{1}, \ldots, i_{\nu}\right) \in \mathscr{X}$ is adapted to $\Omega$. Let $\mathbf{i}^{\prime}=\left(i_{2}, \ldots, i_{\nu}, j\right) \mathscr{X}$ be defined by $s_{j}=w_{0} s_{i_{1}} w_{0}^{-1}$. Then

(a) $\mathbf{i}^{\prime}$ is in $\mathscr{X}$ and is adapted to $s_{i_{1}} \Omega$.

This follows from the proof of 4.12 .

We now prove the following statement.

(b) If $i \rightarrow j$ in $\Omega$ then $\alpha_{j}$ precedes $\alpha_{i}$ in the sequence 4.13(a).

Assume that $\alpha_{i}$ precedes $\alpha_{j}$ in the sequence 4.13(a). If we had $j=i_{1}$, then $\alpha_{j}=\alpha^{1}$ clearly precedes $\alpha_{i}$ and we have a contradiction. Thus, $j \neq i_{1}$. Then $i \rightarrow j$ in $s_{i_{1}} \Omega$. The sequence 4.13(a) has the following form:

$$
\alpha_{i_{1}}, \ldots, \alpha_{i}, \ldots, \alpha_{j}, \ldots
$$

if $a_{i i_{1}}=0$ and

$$
\alpha_{i_{1}}, \ldots, \alpha_{i_{1}}+\alpha_{i}, \ldots, \alpha_{i}, \ldots, \alpha_{j}, \ldots
$$

if $a_{i i_{1}}=-1$. The sequence analogous to $4.13(\mathrm{a})$ corresponding to $\mathrm{i}^{\prime}$ above is

$$
s_{i_{1}}\left(\alpha^{2}\right), s_{i_{1}}\left(\alpha^{3}\right), \ldots, s_{i_{1}}\left(\alpha^{\nu}\right), \alpha^{1}
$$

hence is of the form

if $a_{i i_{1}}=0$ and

$$
\ldots, \alpha_{i}, \ldots, \alpha_{j}, \ldots
$$

$$
\ldots, \alpha_{i}, \ldots, \alpha_{i_{1}}+\alpha_{i}, \ldots, \alpha_{j}, \ldots
$$

if $a_{i i_{1}}=-1$. In both cases we see that $\alpha_{i}$ precedes $\alpha_{j}$ in the sequence analogous to 4.13(a) corresponding to $i^{\prime}$. We can now repeat the previous argument for $\mathbf{i}^{\prime}$ instead of $\mathbf{i}$, etc. Eventually we find a sequence that begins with $j$ and we have a contradiction.

4.15. From 4.12(a) it follows that any indecomposable module in $M \Omega$ remains indecomposable over the algebraic closure of $F$ and that, in fact, the classification of (indecomposable) modules is independent of the ground field $F$. 
It also follows that there is a bijection $\mathbf{c} \rightarrow \mathbf{V}_{\mathbf{c}}$ between $\mathbf{N}^{\nu}$ and the set of isomorphism classes of objects in $M \Omega$, where $\mathbf{V}_{\mathbf{c}}$ is the direct sum of $c_{k}$ copies of $e_{\alpha^{k}}$ for $k=1, \ldots, \nu .\left(\alpha^{k}\right.$ are as in 4.12(c) and $\mathrm{c}=\left(c_{1}, \ldots, c_{\nu}\right)$.)

We have $\operatorname{dim}\left(\mathbf{V}_{\mathbf{c}}\right)=\left(d_{1}, \ldots, d_{n}\right)$ where

$$
\sum_{k=0}^{\nu} c_{k} \alpha^{k}=\sum_{i=0}^{n} d_{i} \alpha_{i}
$$

In particular,

(b) up to isomorphism, there are only finitely many modules in $M \Omega$ of specified dimension $\left(\in \mathbf{N}^{n}\right)$.

4.16. Let $\mathbf{d}=\left(d_{1}, \ldots, d_{n}\right) \in \mathbf{N}^{n}$. Let

$$
\mathbf{E}_{\mathbf{d}}=\bigoplus_{i \rightarrow j} \operatorname{Hom}\left(F^{d_{i}}, F^{d_{j}}\right)
$$

sum over all arrows $i \rightarrow j$ in $\Omega$ and let $G_{\mathbf{d}}=\prod_{i} \mathrm{GL}_{d_{i}}(F)$. The group $G_{\mathbf{d}}$ acts naturally on the $F$-vector space $\mathbf{E}_{\mathbf{d}}$ by $\left(g_{i}\right):\left(f_{i j}\right) \rightarrow\left(g_{j} f_{i j} g_{i}^{-1}\right)$. Any point in $\mathbf{E}_{\mathrm{d}}$ may be regarded as a module in $M \Omega$ of dimension $\mathbf{d}$. Moreover two points in $\mathbf{E}_{\mathbf{d}}$ define isomorphic modules if and only if they are in the same $G_{\mathrm{d}}$-orbit. This gives a 1-1 correspondence between the set of $G_{\mathrm{d}}$-orbits on $\mathbf{E}_{\mathrm{d}}$ and the set of isomorphism classes of modules of dimension $\mathrm{d}$ in $M \Omega$. Let $\mathscr{O}_{\mathrm{c}}$ be the orbit corresponding to the module $\mathbf{V}_{\mathbf{c}}$ (see 4.15). Thus the $G_{\mathrm{d}}$-orbits on $\mathbf{E}_{\mathbf{d}}$ have been indexed by the sequences $\mathbf{c} \in \mathbf{N}^{\nu}$ satisfying 4.15(a). Since there are only finitely many such sequences we see that

(a) if $F$ is algebraically closed, there is a unique open dense $G_{d}$-orbit on $\mathbf{E}_{\mathbf{d}}$.

By a well-known argument of Tits (see [BGP]) we have the following result.

(b) If $F$ is algebraically closed and the $G_{\mathrm{d}}$-orbit $\mathscr{O}$ on $\mathbf{E}_{\mathbf{d}}$ corresponds to an indecomposable module, then $\mathscr{O}$ is the unique open dense orbit.

\section{Multiplication}

5.1. In this section we shall assume that $F=F_{q}$, a finite field with $q$ elements. Let $\Omega$ be an orientation of the Dynkin graph. We fix a sequence $\mathbf{i} \in \mathscr{X}$ adapted to $\Omega$; let $\alpha^{1}, \ldots, \alpha^{\nu}$ be the corresponding total order on $R^{+}$(see 4.13). Following Ringel [R], we define $R \Omega$ to be the $C$-vector space with basis (V) indexed by the isomorphism classes of objects in $M \Omega$ with the $\mathrm{C}$-algebra structure given by

$$
\mathbf{v}^{\prime} \cdot \mathbf{v}^{\prime \prime}=\sum_{\mathbf{v}} g_{\mathbf{v}, \mathbf{v}^{\prime}, \mathbf{v}^{\prime \prime}} \mathbf{V}
$$

where $g_{\mathbf{V}, \mathbf{v}^{\prime}, \mathbf{v}^{\prime \prime}}$ is the number of submodules of $\mathbf{V}$ that are isomorphic to $\mathbf{V}^{\prime \prime}$ and are such that the corresponding quotient module is isomorphic to $\mathbf{V}^{\prime}$. This is an associative algebra with unit element (the 0 module). If $i$ is a sink (resp. a source ) of $\Omega$, we denote by $R_{i}^{+} \Omega$ (resp. $R_{i}^{-} \Omega$ ) the subspace of $R \Omega$ spanned by the $\mathbf{V}$ in $M_{i}^{+} \Omega$ (resp. $M_{i}^{-} \Omega$ ); it is easy to see that this is a subalgebra of $R \Omega$. The following result is easily verified. 
(a) If $i$ is a sink of $\Omega$, then $\Phi_{i}^{+}: M_{i}^{+} \Omega \cong M_{i}^{-} s_{i} \Omega$ defines an algebra isomorphism $R_{i}^{+} \Omega \cong R_{i}^{-} s_{i} \Omega$ with inverse defined by $\Phi_{i}^{-}$.

5.2. If $i \rightarrow j$ is an arrow in $\Omega$, we denote by $e_{i j}$ the module defined by $V_{i}=F, V_{j}=F, V_{h}=0$ for $h \neq i, j, f_{i j}=$ Id. With the notation in 4.12(a), we have $e_{i j}=e_{\alpha_{i}+\alpha_{j}}$. The following identities in $R \Omega$ are easily verified.

$$
e_{i} e_{j}=e_{j} e_{i}+e_{i j}, \quad e_{i} e_{i j}=q e_{i j} e_{i}, \quad e_{i j} e_{j}=q e_{j} e_{i j} .
$$

It follows that

(b) $\quad e_{i}^{2} e_{j}-(q+1) e_{i} e_{j} e_{i}+q e_{j} e_{i}^{2}=0, \quad e_{i} e_{j}^{2}-(q+1) e_{j} e_{i} e_{j}+q e_{j}^{2} e_{i}=0$.

5.3. For any $N \in \mathbf{N}$ and $\alpha \in R^{+}$, let $[[N]] !=\prod_{s=1}^{N} \frac{q^{s}-1}{q-1}$ and

$$
e_{\alpha}^{((N))}=([[N]] !)^{-1} e_{\alpha}^{N} \in R \Omega .
$$

If $P \in M \Omega$ is a direct sum of $N$ copies of $e_{\alpha^{k}}$, then we have as in 4.9(a)

$$
P=\Phi_{i_{1}}^{-} \Phi_{i_{2}}^{-} \cdots \Phi_{i_{k-1}}^{-}(X)
$$

where $X$ is a direct sum of $N$ copies of $e_{i_{k}}$ (in the appropriate category). From the definitions in 5.1 it follows immediately that $X=([[N]] !)^{-1} e_{i_{k}}^{N}$; now using 5.1(a) we deduce that

in $R \Omega$.

$$
P=e_{\alpha^{k}}^{((N))}
$$

Lemma 5.4. (a) The algebra $R \Omega$ is generated by the elements $e_{i}(i \in[1, n])$.

(b) Let $i=i_{1}$. The algebra $R_{i}^{+} \Omega$ is generated by the elements $e_{j}(j \neq i)$ and $e_{j i}(j \rightarrow i)$.

Let $\mathbf{c} \in \mathbf{N}^{\nu}$ and let $\mathbf{V}_{\mathbf{c}} \in M \Omega$ be as in 4.15. By 4.9(b) and 5.3 we have $\mathbf{V}_{\mathbf{c}}=P_{1} P_{2} \cdots P_{\nu}$ (product in $R \Omega$ ) where $P_{k}=e_{\alpha^{k}}^{\left(\left(c_{k}\right)\right)}$; hence

$$
\mathbf{V}_{\mathbf{c}}=e_{\alpha^{1}}^{\left(\left(c_{1}\right)\right)} e_{\alpha^{2}}^{\left(\left(c_{2}\right)\right)} \cdots e_{\alpha^{\nu}}^{\left(\left(c_{\nu}\right)\right)}
$$

(product in $R \Omega$ ).

Consider the partial order on $\mathbf{N}^{\nu}$ defined by $\mathbf{c}^{\prime} \leq \mathbf{c}^{\prime \prime}$ if either

(d) any coordinate of $\operatorname{dim}\left(\mathbf{V}_{\mathbf{c}^{\prime}}\right)$ is $\leq$ than the corresponding coordinate of $\operatorname{dim}\left(\mathbf{V}_{\mathbf{c}^{\prime \prime}}\right)$ and these two vectors do not coincide or

(e) $\operatorname{dim}\left(\mathbf{V}_{\mathbf{c}^{\prime}}\right)=\operatorname{dim}\left(\mathbf{V}_{\mathbf{c}^{\prime}}\right)$ and $\mathscr{O}_{\mathbf{c}^{\prime}}$ is contained in the Zariski closure of $\mathscr{O}_{\mathbf{c}^{\prime \prime}}$ (orbits as in 4.16 over the algebraic closure of $F$ ).

Let $R^{\prime}$ be the subalgebra of $R \Omega$ generated by the elements $e_{i}$. We shall prove that $\mathbf{V}_{\mathbf{c}}$ is contained in $R^{\prime}$. We may assume that the analogous statement for $\mathbf{V}_{\mathbf{c}^{\prime}}$ is already known whenever $\mathbf{c}^{\prime}<\mathbf{c}$.

Using (c), we see that we may assume that $\mathbf{V}_{\mathrm{c}}=e_{\alpha^{k}}$ for some $k$. We can find $h \in[1, n]$ such that $\mathbf{V}_{\mathbf{c}}$ is nonzero at $h$ and such that for any arrow $h \rightarrow h^{\prime}$ in $\Omega$ the linear map $f_{h h^{\prime}}$ (in the module structure of $\mathbf{V}_{\mathbf{c}}$ ) is zero. (We 
use the fact that the Dynkin graph has no cycles.) Let $Y$ be the module whose $h$-component is equal to the $h$-component of $\mathbf{V}_{\mathbf{c}}$ and whose other components are zero. We have a canonical injective morphism $Y \rightarrow \mathbf{V}_{\mathbf{c}}$ with cokernel $Y^{\prime}$. It is clear that the product $Y^{\prime} Y$ in $R \Omega$ is equal to $\mathbf{V}_{\mathrm{c}}$ plus a linear combination of elements $\mathbf{V}_{\mathbf{c}^{\prime}}$ such that $\operatorname{dim}\left(\mathbf{V}_{\mathbf{c}^{\prime}}\right)=\operatorname{dim}\left(\mathbf{V}_{\mathbf{c}^{\prime}}\right)$ and $\mathbf{c}^{\prime} \neq \mathbf{c}$.

All these $\mathbf{c}^{\prime}$ satisfy $\mathbf{c}^{\prime}<\mathbf{c}$, since, by $4.16(\mathrm{~b}), \mathscr{O}_{\mathbf{c}}$ is an open orbit. By the induction hypothesis, we have $\mathbf{V}_{\mathbf{c}^{\prime}} \in R^{\prime}$ for all such $\mathbf{c}^{\prime}$. It remains to show that $Y^{\prime}$ and $Y$ are in $R^{\prime}$. If $Y^{\prime} \neq 0$, this again follows from the induction hypothesis. Hence we may assume that $Y^{\prime}=0$ and $\mathbf{v}_{c}=Y$. Since $\mathbf{v}_{c}$ is indecomposable, it is equal to $e_{h}$, which is contained in $R^{\prime}$ by definition. This proves (a).

We now prove (b). Let $R^{\prime \prime}$ be the subalgebra of $R_{i}^{+} \Omega$ generated by the elements indicated in (b). We have $\mathbf{V}_{\mathbf{c}} \in M_{i}^{+} \Omega$ if and only if $c_{i_{1}}=0$. Assuming that $c_{i_{1}}=0$, we shall prove that $\mathbf{V}_{\mathbf{c}}$ is contained in $R^{\prime \prime}$. We may assume that the analogous statement for $\mathbf{V}_{\mathbf{c}^{\prime}}$ is already known whenever $\mathbf{c}^{\prime}<\mathbf{c}$ and $c_{i_{1}}^{\prime}=0$. Using again (c), we see that we may assume that $\mathbf{V}_{\mathbf{c}}=e_{\alpha^{k}}$ for some $k>1$. Let $h, Y, Y^{\prime}$ be as in the first part of the proof. We have $Y^{\prime} \in M_{i}^{+} \Omega$; if moreover $h \neq i$, we have also $Y \in M_{i}^{+} \Omega$ and our earlier argument gives the desired conclusion. Hence we can assume that the following condition is satisfied:

(f) for any $h \in[1, n]$ such that $h \neq i$ and such that $\mathbf{V}_{\mathbf{c}}$ is nonzero at $h$ there exists some arrow $h \rightarrow h^{\prime}$ in $\Omega$ such that the linear map $f_{h h^{\prime}}$ (in the module structure of $\mathbf{V}_{\mathbf{c}}$ ) is nonzero.

Since $k>1$, the $j$-component of $\mathbf{V}_{\mathbf{c}}$ is nonzero for some $j \neq i$. Using the fact that the Dynkin graph has no cycles, we see that we can find such a $j$ such that, in addition, for any arrow $j \rightarrow h^{\prime}$ in $\Omega$, with $h^{\prime} \neq i$, the linear map map $f_{j h^{\prime}}$ (in the module structure of $\mathbf{V}_{\mathbf{c}}$ ) is zero. From (f) it then follows that $f_{j i}$ (in the module structure of $\mathbf{V}_{\mathbf{c}}$ ) is nonzero. Let $Y$ be the submodule of $\mathbf{V}_{\mathbf{c}}$ consisting of the $j$-component of $\mathbf{V}_{\mathbf{c}}$ and its image under $f_{j i}$ (at the vertex $i$ ). It is clear that this is well defined and that it belongs to $M_{i}^{+} \Omega$. Let $Y^{\prime}$ be the corresponding quotient module. We have automatically $Y^{\prime} \in M_{i}^{+} \Omega$.

We again have that the product $Y^{\prime} Y$ in $R_{i}^{+} \Omega$ is equal to $\mathbf{V}_{\mathrm{c}}$ plus a linear combination of elements $\mathbf{V}_{\mathbf{c}^{\prime}}$ such that $c_{i_{1}}^{\prime} \neq 0, \operatorname{dim}\left(\mathbf{V}_{\mathbf{c}^{\prime}}\right)=\operatorname{dim}\left(\mathbf{V}_{\mathbf{c}}\right)$ and $\mathbf{c}^{\prime}<\mathbf{c}$.

By the induction hypothesis, we have $\mathbf{V}_{\mathbf{c}^{\prime}} \in R^{\prime \prime}$ for all such $\mathbf{c}^{\prime}$. It remains to show that $Y^{\prime}$ and $Y$ are in $R^{\prime \prime}$. If $Y^{\prime} \neq 0$, this again follows from the induction hypothesis. Hence we may assume that $Y^{\prime}=0$ and $\mathbf{V}_{\mathbf{c}}=Y$. Since $\mathbf{V}_{\mathrm{c}}$ is indecomposable, it is equal to $e_{j i}$, which is contained in $R^{\prime \prime}$ by definition. This proves (b).

5.5. We regard $\mathrm{C}$ as an $A$-algebra with $v$ acting as multiplication by a fixed square root $q^{1 / 2}$ of $q$. Let $U_{q}, U_{q}^{+}$be the $\mathbf{C}$-algebras obtained from the $A$ algebras $U, U^{+}$by extension of scalars. Let $\mathbf{z}=\left(z_{1}, \ldots, z_{n}\right) \in \mathbf{Z}^{n}$ be such 
that $z_{i}-z_{j}=1$ whenever $i \rightarrow j$ is an arrow in $\Omega$. Such $\mathbf{z}$ clearly exists.

5.6. Let $U_{q}^{0}$ be the subalgebra of $U_{q}$ generated by $K_{i}, K_{i}^{-1}$ for all $i$. It is isomorphic to the the group algebra of $\mathbf{Z}^{n}$. Let $\widetilde{R \Omega}=U_{q}^{0} \otimes_{\mathbf{C}} R \Omega$. This may be regarded as an associative algebra containing $U_{q}^{0}$ and $R \Omega$ as subalgebras, with the commutation rule $K_{i} \mathbf{V} K_{i}^{-1}=q^{\sum_{j} a_{i j} d_{j} / 2} \mathbf{V}$ where $\mathbf{V} \in M \Omega$ has dimension $\left(d_{1}, \ldots, d_{n}\right)$. Let $U_{q}^{\geq 0}=U_{q}^{0} \otimes_{c} U_{q}^{+}$; this may be identified with the subalgebra of $U_{q}$ generated by $U_{q}^{0}$ and $U_{q}^{+}$.

Proposition 5.7 (Ringel) [R]. There is a unique algebra isomorphism $U_{q}^{\geq 0} \rightarrow \widetilde{R \Omega}$ such that $K_{i} \rightarrow K_{i}, K_{i}^{-1} \rightarrow K_{i}^{-1}, E_{i} \rightarrow K_{i}^{z_{i}} e_{i}$.

The formulas above define an algebra homomorphism: we must verify that the relations $1.2(\mathrm{a} 1),(\mathrm{a} 2),(\mathrm{a} 3)$, which define the algebra $U_{q}^{\geq 0}$, are respected; this follows easily from $5.2(\mathrm{~b})$. This homomorphism is surjective by $5.4(\mathrm{a})$. For any $\mathbf{d} \in \mathbf{N}^{n}$, we define $U_{\mathbf{d}, q}^{+} \subset U_{q}^{+}$and $R \Omega_{\mathbf{d}}$ in the same way as $\mathbf{U}_{\mathbf{d}}^{+}$was defined in 2.7. These are finite-dimensional vector spaces (of the same dimension) that form a direct sum decomposition of $U_{q}^{+}$and $R \Omega$. Tensoring with $U_{q}^{0}$ we obtain direct sum decompositions of $U_{q}^{\geq 0}$ and $\widetilde{R \Omega}$ into summands (indexed by $\mathbf{N}^{n}$ ).

It is clear that our homomorphism respects these direct sum decompositions. Its restriction to any summand is a surjective homomorphism between free modules of the same finite rank over $U_{q}^{0}$ hence is an isomorphism. The proposition follows.

5.8. The inverse of the isomorphism in 5.7 may be restricted to $R \Omega$ and thus defines an imbedding of algebras $\Gamma: R \Omega \hookrightarrow U_{q}$ such that $\Gamma\left(e_{j}\right)=K_{j}^{-z_{j}} E_{j}$ for all $j \in[1, n]$.

The following property of $\Gamma$ follows easily from the definition.

(a) If $\mathbf{V} \in M \Omega$ has dimension $\left(d_{1}, \ldots, d_{n}\right)$, then $\Gamma(\mathbf{V})=K_{1}^{-z_{1} d_{1}} \cdots K_{n}^{-z_{n} d_{n}} \phi$ where $\phi \in U_{q}^{+}$.

Similarly, if $i=i_{1}$, we have an imbedding of algebras $\Gamma^{\prime}: R s_{i} \Omega \hookrightarrow U_{q}$ such that $\Gamma\left(e_{j}\right)=K_{j}^{-z_{j}^{\prime}} E_{j}$ for all $j \in[1, n]$, where $z_{j}^{\prime}=z_{j}$ for $j \neq i$ and $z_{i}^{\prime}=$ $z_{i}+2$. (Note that $\left(z_{1}^{\prime}, \ldots, z_{n}^{\prime}\right)$ plays the same role for $s_{i} \Omega$ as $\left(z_{1}, \ldots, z_{n}\right)$ for $\Omega$.) Let $\rho_{i}: \mathbf{U} \rightarrow \mathbf{U}$ be the algebra isomorphism defined by

$$
K_{j} \rightarrow K_{j}, \quad E_{j} \rightarrow(-1)^{a_{i j}} v^{a_{i j} z_{i}-\delta_{i j}} K_{i}^{a_{i j}} E_{j}, \quad F_{j} \rightarrow(-1)^{a_{i j}} v^{-a_{i j} z_{i}+\delta_{i j}} F_{j} K_{i}^{-a_{i j}}
$$

The same formula (with $v=q^{1 / 2}$ ) defines an algebra isomorphism $\rho_{i}: U_{q} \rightarrow$ $U_{q}$. With these notations we can state the following result.

Lemma 5.9. For any $\xi \in R_{i}^{-} s_{i} \Omega$ we have $T_{i}^{-1}\left(\rho_{i}\left(\Gamma^{\prime}(\xi)\right)\right)=\Gamma\left(\Phi_{i}^{-}(\xi)\right)$ where $T_{i}^{-1}$ is as in 1.3 . 
We set $v=q^{1 / 2}$. Using 5.4(b) and 5.1(a) we see that the algebra $R_{i}^{-} s_{i} \Omega$ is generated by the elements $e_{j}(j \neq i)$ and $e_{i j}\left(i \rightarrow j\right.$ in $\left.s_{i} \Omega\right)$. Hence we may assume that $\xi$ is one of these generators. Assume first that $\xi=e_{j}$ where $a_{i j}=-1$. Then $z_{i}=z_{j}-1$ and

$$
\begin{aligned}
\Gamma\left(\Phi_{i}^{-}(\xi)\right)=\Gamma\left(e_{j i}\right) & =\Gamma\left(e_{j} e_{i}-e_{i} e_{j}\right)=K_{j}^{-z_{j}} E_{j} K_{i}^{-z_{i}} E_{i}-K_{i}^{-z_{i}} E_{i} K_{j}^{-z_{j}} E_{j} \\
= & -v^{-z_{i}} K_{j}^{-z_{j}} K_{i}^{-z_{i}}\left(-E_{j} E_{i}+v^{-1} E_{i} E_{j}\right), \\
T_{i}^{-1}\left(\rho_{i}\left(\Gamma^{\prime}(\xi)\right)\right) & =T_{i}^{-1}\left(\rho_{i}\left(K_{j}^{-z_{j}} E_{j}\right)\right)=T_{i}^{-1}\left(-v^{-z_{i}} K_{i}^{-1} K_{j}^{-z_{j}} E_{j}\right) \\
& =-v^{-z_{i}} K_{j}^{-z_{j}} K_{i}^{-z_{i}}\left(-E_{j} E_{i}+v^{-1} E_{i} E_{j}\right)
\end{aligned}
$$

as required. Assume next that $\xi=e_{j}$ where $a_{i j}=0$. Then

$$
\begin{gathered}
\Gamma\left(\Phi_{i}^{-}(\xi)\right)=\Gamma\left(e_{j}\right)=K_{j}^{-z_{j}} E_{j}, \\
T_{i}^{-1}\left(\rho_{i}\left(\Gamma^{\prime}(\xi)\right)\right)=T_{i}^{-1}\left(\rho_{i}\left(K_{j}^{-z_{j}} E_{j}\right)\right)=K_{j}^{-z_{j}} E_{j}
\end{gathered}
$$

as required. Finally, assume that $\xi=e_{i j}=e_{i} e_{j}-e_{j} e_{i}$ where $i \rightarrow j$ in $s_{i} \Omega$. Then $z_{i}=z_{j}-1$ and

$$
\begin{aligned}
& \Gamma\left(\Phi_{i}^{-}(\xi)\right)=\Gamma\left(e_{j}\right)=K_{j}^{-z_{j}} E_{j} \\
T_{i}^{-1}\left(\rho_{i}\left(\Gamma^{\prime}(\xi)\right)\right)= & T_{i}^{-1}\left(\rho_{i}\left(K_{i}^{-z_{i}-2} E_{i} K_{j}^{-z_{j}} E_{j}-K_{j}^{-z_{j}} E_{j} K_{i}^{-z_{i}-2} E_{i}\right)\right) \\
= & T_{i}^{-1}\left(\rho_{i}\left(K_{i}^{-z_{i}-2} K_{j}^{-z_{j}}\left(v^{-z_{j}} E_{i} E_{j}-v^{-z_{i}-2} E_{j} E_{i}\right)\right)\right) \\
= & K_{i} K_{j}^{-z_{j}} v^{-z_{j}} T_{i}^{-1}\left(\rho_{i}\left(E_{i} E_{j}-v^{-1} E_{j} E_{i}\right)\right) \\
= & K_{i} K_{j}^{-z_{j}} v^{-z_{j}} v^{z_{i}+1} K_{i}^{-1} E_{j}=K_{j}^{-z_{j}} E_{j}
\end{aligned}
$$

as required. The lemma is proved.

5.10. Let $k \in[1, \nu]$. As in 5.8 , we have an algebra homomorphism $\Gamma_{k}: R \Omega_{k}$ $\hookrightarrow U_{q}$ such that $\Gamma_{k}\left(e_{j}\right)=K_{j}^{-z_{j}^{k}} E_{j}$ for $j \in[1, n]$ where $\Omega_{k}$ is as in 4.7 and $z_{j}^{k}$ are defined inductively by $z_{j}^{1}=z_{j}, z_{j}^{k}=z_{j}^{k-1}$ for $k>1, j \neq i_{k-1}$, $z_{j}^{k}=z_{j}^{k-1}+2$ for $k>1, j=i_{k-1}$. Let $k^{\prime} \in[1, k]$. The following identity holds in $U_{q}$.

$$
\Gamma_{k^{\prime}}\left(\boldsymbol{\Phi}_{i_{k^{\prime}}}^{-} \cdots \boldsymbol{\Phi}_{i_{k-2}}^{-} \Phi_{i_{k-1}}^{-}\left(e_{i_{k}}\right)\right)=q^{f \prime / 2} K^{\prime} \tilde{T}_{i_{k^{\prime}}} \cdots \tilde{T}_{i_{k-2}} \tilde{T}_{i_{k-1}}\left(E_{i_{k}}\right)
$$

where $\widetilde{T}_{i}$ are as in 1.3, $e_{i_{k}}$ is regarded as an element of $R \Omega_{k}, f^{\prime}$ is an integer and $K^{\prime}$ is a monomial in the $K_{i}, K_{i}^{-1}$. This is proved by descending induction on $k^{\prime}$, using 5.9 repeatedly.

For $k^{\prime}=1$, the last identity can be written in the form

$$
\Gamma\left(e_{\alpha^{k}}\right)=q^{f^{\prime} / 2} K^{\prime} \widetilde{T}_{i_{1}} \cdots \widetilde{T}_{i_{k-2}} \tilde{T}_{i_{k-1}}\left(E_{i_{k}}\right)
$$


where $f^{\prime}$ is an integer and $K^{\prime}$ is a monomial in the $K_{i}, K_{i}^{-1}$. (Here, $e_{\alpha^{k}}$ is regarded as an element of $R \Omega$.) If $V_{\mathrm{c}} \in M \Omega$ are as in 4.15 and $E_{\mathrm{i}}^{\mathrm{c}} \in U^{+}$(or in $U_{q}^{+}$) are as in $2.2(\mathrm{a})$, we have (using (a) and 5.4(c))

$$
\Gamma\left(V_{\mathbf{c}}\right)=q^{f_{\mathbf{c}} / 2} K E_{\mathbf{i}}^{\mathbf{c}} \in U_{q}
$$

where $f_{\mathrm{c}}$ is an integer and $K$ is a monomial in the $K_{i}, K_{i}^{-1}$, depending on $\mathbf{c} \in \mathbf{N}^{\nu}$. More precisely, if $\operatorname{dim}(\mathbf{V})=\mathbf{d}=\left(d_{1}, \ldots, d_{n}\right)$ and $K(\mathbf{d})=$ $K_{1}^{-z_{1} d_{1}} \cdots K_{n}^{-z_{n} d_{n}}$, then

$$
K=K(\mathbf{d})
$$

as we see from $5.8(a)$.

5.11. The integers $f_{\mathbf{c}}$ will be described in $\S 7$. Here we note only that they are independent of $q$ and that they satisfy a certain identity, which we now explain. Let $\mathbf{c}=\left(c_{1}, \ldots, c_{\nu}\right) \in \mathbf{N}^{\nu}$ be of i-homogeneity $\mathbf{d}$. Let $\mathbf{c}_{k} \in \mathbf{N}^{\nu}$ be such that its $k$ th coordinate is $c_{k}$ and its other coordinates are zero. Let $\mathbf{d}^{k}=\left(d_{1}^{k}, \ldots, d_{n}^{k}\right)$ be the i-homogeneity oi $\mathbf{c}_{k}$. We have

$$
\Gamma\left(\mathbf{V}_{\mathbf{c}}\right)=\Gamma\left(\mathbf{V}_{\mathbf{c}_{1}} \cdots \mathbf{V}_{\mathbf{c}_{\nu}}\right)=\Gamma\left(\mathbf{V}_{\mathbf{c}_{1}}\right) \cdots \Gamma\left(\mathbf{V}_{\mathbf{c}_{\nu}}\right) .
$$

Using $5.10(\mathrm{~b})$, this can be written as

$$
q^{f_{\mathrm{c}} / 2} K(\mathbf{d}) E_{\mathbf{i}}^{\mathbf{c}}=q^{f_{\mathbf{c}_{1}} / 2} K\left(\mathbf{d}^{1}\right) E_{\mathbf{i}}^{\mathbf{c}_{1}} \cdots q^{f_{\mathfrak{c}_{\nu}} / 2} K\left(\mathbf{d}^{\nu}\right) E_{\mathbf{i}}^{\mathbf{c}_{\nu}}
$$

Using the commutation formulas

$$
E_{\mathbf{i}}^{\mathbf{c}^{\prime}} K\left(\mathbf{d}^{k}\right)=q^{m_{k^{\prime}, k} / 2} K\left(\mathbf{d}^{k}\right) E_{\mathbf{i}}^{\mathbf{c}^{\prime}} \quad\left(k^{\prime}<k\right)
$$

where $m_{k^{\prime}, k}=\sum_{i} z_{i} d_{i}^{k} c_{k^{\prime}}\left(\alpha_{i}, \alpha^{k^{\prime}}\right)$, we move all $K$-factors in (a) to the left and we deduce

$$
f_{\mathbf{c}}=\sum_{k} f_{\mathbf{c}_{k}}+\sum_{k^{\prime}<k ; i} z_{i} d_{i}^{k} c_{k^{\prime}}\left(\alpha_{i}, \alpha^{k^{\prime}}\right) \text {. }
$$

We now substitute $c_{k^{\prime}}\left(\alpha_{i}, \alpha^{k^{\prime}}\right)=\sum_{j} a_{i j} d_{j}^{k^{\prime}}$ and we obtain

$$
f_{\mathbf{c}}=\sum_{k} f_{\mathbf{c}_{k}}+\sum_{k^{\prime}<k ; i, j} a_{i j} z_{i} d_{i}^{k} d_{j}^{k^{\prime}} \text {. }
$$

Lemma 5.12 (Ringel). For any $\mathbf{c}, \mathbf{c}^{\prime}, \mathbf{c}^{\prime \prime}$ in $\mathbf{N}^{\nu}, g_{\mathbf{v}_{\mathbf{c}}}, \mathbf{v}_{\mathbf{c}^{\prime}}, \mathbf{v}_{\mathbf{c}^{\prime \prime}}$ is, as a function of $q$, given by a polynomial with integer coefficients in $q$.

For a proof, see $[R]$.

\section{DIMENSION OF ORBITS}

6.1. In this section, unless otherwise specified, $F$ is an algebraic closure of a finite field $F_{q}$. We again fix an orientation $\Omega$ for our Dynkin graph, an 
$\mathbf{i} \in \mathscr{X}$ adapted to $\Omega$ and we consider the positive roots ordered accordingly. Let $\mathbf{d}, \mathbf{d}^{\prime}, \mathbf{d}^{\prime \prime} \in \mathbf{N}^{n}$ be such that $\mathbf{d}=\mathbf{d}^{\prime}+\mathbf{d}^{\prime \prime}$. We define a diagram of varieties

$$
\mathbf{E}_{\mathbf{d}^{\prime}} \times \mathbf{E}_{\mathbf{d}^{\prime \prime}} \stackrel{\beta}{\longleftarrow} \mathbf{E}^{\prime} \stackrel{\beta^{\prime}}{\longrightarrow} \mathbf{E}^{\prime \prime} \stackrel{\beta^{\prime \prime}}{\longrightarrow} \mathbf{E}_{\mathbf{d}}
$$

as follows. $\mathbf{E}_{\mathbf{d}}, \mathbf{E}_{\mathbf{d}^{\prime}}, \mathbf{E}_{\mathbf{d}^{\prime \prime}}$ are as in 4.16. A point of $\mathbf{E}^{\prime \prime}$ is by definition a point $\left(f_{i j}\right)_{i \rightarrow j}$ of $\mathbf{E}_{\mathbf{d}}$ together with a $d_{i}^{\prime \prime}$-dimensional subspace $C_{i}$ of $F^{d_{i}}$ for each $i \in[1, n]$ such that $f_{i j}\left(C_{i}\right) \subset C_{j}$ for any $i \rightarrow j$ in $\Omega$. The map $\beta^{\prime \prime}$ is the obvious one (forgetting the $C_{i}$ 's). A point of $\mathbf{E}^{\prime}$ is by definition a point $\left(f_{i j}, C_{i}\right)$ of $\mathbf{E}^{\prime \prime}$ together with a collection of isomorphisms $\mu_{i}^{\prime \prime}: F^{d_{i}^{\prime \prime}} \cong C_{i}$, $\mu_{i}^{\prime}: F^{d_{i}} / C_{i} \cong F^{d_{i}^{\prime}}$ for $i \in[1, n]$. To this point we attach the point $\left(f_{i j}^{\prime \prime}\right)_{i \rightarrow j}$ of $\mathbf{E}_{\mathbf{d}^{\prime \prime}}$ and the point $\left(f_{i j}^{\prime}\right)_{i \rightarrow j}$ of $\mathbf{E}_{\mathbf{d}^{\prime}}$ by $f_{i j}^{\prime \prime}=\left(\mu_{j}^{\prime \prime}\right)^{-1} f_{i j} \mu_{i}^{\prime \prime}, f_{i j}^{\prime}=\mu_{j}^{\prime} f_{i j}\left(\mu_{i}^{\prime}\right)^{-1}$ (the maps $C_{i} \rightarrow C_{j}$ and $F^{d_{i}} / C_{i} \rightarrow F^{d_{j}} / C_{j}$ defined by $f_{i j}$ are denoted again by $f_{i j}$ ). This defines the map $\beta$. The map $\beta^{\prime}$ is the obvious one (forgetting the $\left.\mu_{i}^{\prime \prime}, \mu_{i}^{\prime}\right)$. The group $G_{\mathrm{d}} \times G_{\mathrm{d}^{\prime}} \times G_{\mathrm{d}^{\prime \prime}}$ (see 4.16) acts naturally on each of the varieties in (a) so that the maps in (a) are compatible with this action. The action on $\mathbf{E}_{\mathrm{d}^{\prime}} \times \mathbf{E}_{\mathrm{d}^{\prime \prime}}$ is as in 4.16 on the factors $G_{\mathrm{d}^{\prime}}, G_{\mathrm{d}^{\prime \prime}}$ and $G_{\mathrm{d}}$ acts trivially. The action on $\mathbf{E}_{\mathbf{d}}$ is as in 4.16 on the factor $G_{\mathrm{d}}$ and the other two factors act trivially.

(b) The map $\beta^{\prime}$ is a principal $G_{\mathrm{d}^{\prime}} \times G_{\mathrm{d}^{\prime \prime}}$ fibration.

(c) The map $\beta$ is a locally trivial fibration with smooth connected fibres of dimension

$$
\sum_{i} d_{i}^{\prime 2}+\sum_{i}\left(d_{i}^{\prime \prime}\right)^{2}+\sum_{i} d_{i}^{\prime} d_{i}^{\prime \prime}+\sum_{i \rightarrow j} d_{i}^{\prime} d_{j}^{\prime \prime}
$$

(d) The map $\beta^{\prime \prime}$ is proper.

6.2. Now let $\mathscr{O}_{\mathbf{c}^{\prime}}$ be a $G_{\mathbf{d}^{\prime}}$-orbit on $\mathbf{E}_{\mathbf{d}^{\prime}}$ and let $\mathscr{O}_{\mathbf{c}^{\prime \prime}}$ be a $G_{\mathbf{d}^{\prime \prime}}$-orbit on $\mathbf{E}_{\mathbf{d}^{\prime \prime}}$ (notation of 4.16).

Let $Z=\beta^{\prime}\left(\beta^{-1}\left(\mathscr{O}_{\mathbf{c}^{\prime}} \times \mathscr{O}_{\mathbf{c}^{\prime \prime}}\right)\right)$. From $6.1(\mathrm{~b}),(\mathrm{c})$, we see that $Z$ is a smooth, connected variety of dimension

$$
\operatorname{dim} Z=\operatorname{dim}\left(\mathscr{O}_{\mathbf{c}^{\prime}}\right)+\operatorname{dim}\left(\mathscr{O}_{\mathbf{c}^{\prime \prime}}\right)+\sum_{i} d_{i}^{\prime} d_{i}^{\prime \prime}+\sum_{i \rightarrow j} d_{i}^{\prime} d_{j}^{\prime \prime}
$$

Now let $\mathscr{O}_{\mathrm{c}}$ be a $G_{\mathrm{d}}$-orbit on $\mathbf{E}_{\mathrm{d}}$.

It follows easily from the definitions $6.1,4.5$ that the following two conditions are equivalent:

(b) $\mathbf{V}_{\mathrm{c}} \cong \mathbf{V}_{\mathbf{c}^{\prime}} \star \mathbf{V}_{\mathbf{c}^{\prime \prime}}$;

(c) $\beta^{\prime \prime}$ restricts to a bijection $Z \cong \mathscr{O}_{c}$

(notation of 4.15). Hence, if (b) holds, we have

$$
\operatorname{dim}\left(\mathscr{O}_{\mathbf{c}}\right)=\operatorname{dim}\left(\mathscr{O}_{\mathbf{c}^{\prime}}\right)+\operatorname{dim}\left(\mathscr{O}_{\mathbf{c}^{\prime \prime}}\right)+\sum_{i} d_{i}^{\prime} d_{i}^{\prime \prime}+\sum_{i \rightarrow j} d_{i}^{\prime} d_{j}^{\prime \prime} .
$$


In general, $\beta^{\prime \prime}(Z)$ is a union of $G_{\mathbf{d}}$-orbits on $\mathbf{E}_{\mathbf{d}}$. We shall write $\left(\mathscr{O}_{\mathbf{c}^{\prime}}, \mathscr{O}_{\mathbf{c}^{\prime \prime}}\right)$ $\Longrightarrow \mathscr{O}_{c}$ whenever $\mathscr{O}_{\mathrm{c}}$ is contained in $\beta^{\prime \prime}(Z)$. In this case, we have clearly

$$
\operatorname{dim}\left(\mathscr{O}_{\mathbf{c}}\right) \leq \operatorname{dim}\left(\mathscr{O}_{\mathbf{c}^{\prime}}\right)+\operatorname{dim}\left(\mathscr{O}_{\mathbf{c}^{\prime \prime}}\right)+\sum_{i} d_{i}^{\prime} d_{i}^{\prime \prime}+\sum_{i \rightarrow j} d_{i}^{\prime} d_{j}^{\prime \prime}
$$

6.3. More generally, suppose that we are given $\mathbf{d}^{k}=\left(d_{1}^{k}, \ldots, d_{n}^{k}\right) \in \mathbf{N}^{n}$ and $\mathbf{c}_{k} \in \mathbf{N}^{\nu}\left(k \in\left[1, k_{0}\right]\right)$ such that $\operatorname{dim} V_{\mathbf{c}_{k}}=\mathbf{d}^{k}$ for all $k$. We say that

$$
\left(\mathscr{O}_{c_{1}}, \mathscr{O}_{c_{2}}, \ldots, \mathscr{O}_{c_{k_{0}}}\right) \Longrightarrow \mathscr{O}_{c}
$$

if there is a sequence $\mathbf{c}^{1}, \mathbf{c}^{2}, \ldots, \mathbf{c}^{k_{0}}$ of elements of $\mathbf{N}^{\nu}$ such that $\mathbf{c}^{1}=\mathbf{c}$, $\mathbf{c}^{k_{0}}=\mathbf{c}_{k_{0}}$ and

$$
\left(\mathscr{O}_{\mathbf{c}_{k}}, \mathscr{O}_{\mathbf{c}^{k+1}}\right) \Longrightarrow \mathscr{O}_{\mathbf{c}^{k}}
$$

for $k \in\left[1, k_{0}-1\right]$.

Lemma 6.4. Assume that either condition 6.3(a) or condition (a) below is satisfied:

$$
\mathbf{V}_{\mathbf{c}} \cong \mathbf{V}_{\mathbf{c}_{1}} \star\left(\mathbf{V}_{\mathbf{c}_{2}} \star\left(\cdots \star \mathbf{V}_{\mathbf{c}_{k_{0}}}\right) \ldots\right)
$$

Then we have

$$
\operatorname{dim}\left(\mathscr{O}_{\mathbf{c}}\right) \leq \sum_{k=1}^{k_{0}} \operatorname{dim}\left(\mathscr{O}_{\mathbf{c}_{k}}\right)+\sum_{h<k ; i} d_{i}^{h} d_{i}^{k}+\sum_{h<k ; i \rightarrow j} d_{i}^{h} d_{j}^{k}
$$

with equality if (a) holds.

For $k_{0}=2$ this is just $6.2(\mathrm{~d}),(\mathrm{e})$; the general case follows by applying repeatedly $6.2(\mathrm{~d}),(\mathrm{e})$.

6.5. We now take $k_{0}=\nu$ and assume that for any $k \in[1, \nu], \mathbf{c}_{k}$ has the same $k$ th coordinate as $\mathbf{c}$ and all its other coordinates are zero. We then have the following result.

\section{Proposition 6.6.}

$$
\operatorname{dim}\left(\mathscr{O}_{\mathbf{c}}\right)=\sum_{k ; i \rightarrow j} d_{i}^{k} d_{j}^{k}+\sum_{h<k ; i} d_{i}^{h} d_{i}^{k}+\sum_{h<k ; i \rightarrow j} d_{i}^{h} d_{j}^{k} .
$$

This can be deduced from 6.4. The condition 6.4(a) is satisfied (see 4.9(b)). Moreover, it is clear that $\operatorname{dim}\left(\mathbf{E}_{\mathbf{c}_{k}}\right)=\sum_{i \rightarrow j} d_{i}^{k} d_{j}^{k}$. Hence it is enough to show that $\operatorname{dim}\left(\mathscr{O}_{\mathbf{c}_{k}}\right)=\operatorname{dim}\left(\mathbf{E}_{\mathbf{c}_{k}}\right)$. But this is contained in the following lemma, which will be proved in 6.8 .

Lemma 6.7. $\mathscr{O}_{\mathbf{c}_{k}}$ is open in $\mathbf{E}_{\mathbf{c}_{k}}$.

6.8. In the setup of $6.1,6.2$, let $Z_{\mathbf{c}, \mathbf{c}^{\prime}, \mathbf{c}^{\prime \prime}}$ be the intersection $Z \cap \beta^{\prime \prime-1} \mathscr{O}_{\mathbf{c}}$. All the varieties considered above are naturally defined over $F_{q}$. It follows easily 
from the definitions that

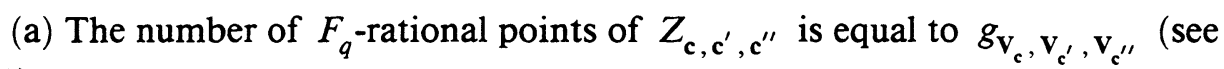
5.1).

(In the last expression, the notation is relative to $F_{q}$.) In particular,

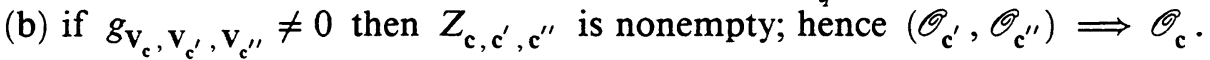

We now take $\mathbf{c}^{\prime}, \mathbf{c}^{\prime \prime}$ to have all coordinates equal to 0 except the $k$ th, which is $a^{\prime}, a^{\prime \prime}$ respectively. If $\mathbf{p}=\left(p_{1}^{k}, \ldots, p_{n}^{k}\right), \alpha^{k}=\sum_{i} p_{i}^{k} \alpha_{i}$, then $\operatorname{dim} \mathbf{V}_{\mathbf{c}^{\prime}}=$

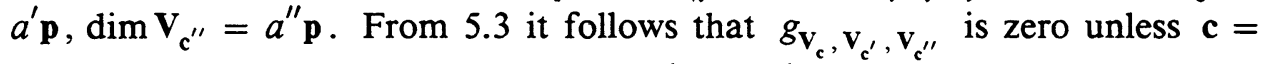
$\mathbf{c}^{\prime}+\mathbf{c}^{\prime \prime}$ in which case it is equal to $[[a]]^{-1}\left[\left[a^{\prime}\right]\right]^{-1}\left[\left[a+a^{\prime}\right]\right]$, a polynomial in $q$ of degree $a^{\prime} a^{\prime \prime}$. Since this holds for any $q$, we see from (a) that $Z_{\mathbf{c}, \mathbf{c}^{\prime}, \mathbf{c}^{\prime \prime}}$ is empty unless $\mathbf{c}=\mathbf{c}^{\prime}+\mathbf{c}^{\prime \prime}$ in which case it is a variety of dimension $a^{\prime} a^{\prime \prime}$. If we now take $\mathbf{c}=\mathbf{c}^{\prime}+\mathbf{c}^{\prime \prime}$, we see that $\operatorname{dim} Z=\operatorname{dim} \mathscr{O}_{\mathbf{c}}+a^{\prime} a^{\prime \prime}$. Using the formula 6.2(a), we deduce

$$
\operatorname{dim} \mathscr{O}_{\mathbf{c}}=\operatorname{dim} \mathscr{O}_{\mathbf{c}^{\prime}}+\operatorname{dim} \mathscr{O}_{\mathbf{c}^{\prime \prime}}+a^{\prime} a^{\prime \prime}\left(-1+\sum_{i} p_{i}^{k} p_{i}^{k}+\sum_{i \rightarrow j} p_{i}^{k} p_{j}^{k}\right)
$$

Since $\left(\alpha^{k}, \alpha^{k}\right)=2$, we have $-1+\sum_{i} p_{i}^{k} p_{i}^{k}=\sum_{i \rightarrow j} p_{i}^{k} p_{j}^{k}$. Introducing this in (c), we obtain

$$
\operatorname{dim} \mathscr{O}_{\mathbf{c}}=\operatorname{dim} \mathscr{O}_{\mathbf{c}^{\prime}}+\operatorname{dim} \mathscr{O}_{\mathbf{c}^{\prime \prime}}+2 a^{\prime} a^{\prime \prime} \sum_{i \rightarrow j} p_{i}^{k} p_{j}^{k}
$$

We want to show that $\operatorname{dim} \mathscr{O}_{\mathbf{c}}=\operatorname{dim} \mathbf{E}_{\mathbf{c}}$. We do this by induction on $a$, the $k$ th coordinate of $\mathbf{c}$. (Recall that the other coordinates are zero.) When $a=1$, this follows from 4.16(b). We can assume that $a>1$ and that our statement is already known when $a$ is replaced by a smaller number. Consider the identity (d) with $a=a^{\prime}+a^{\prime \prime}, a^{\prime}<a, a^{\prime \prime}<a$. Using the induction hypothesis, we have

$$
\operatorname{dim} \mathscr{O}_{\mathbf{c}}=\operatorname{dim} \mathbf{E}_{\mathbf{c}^{\prime}}+\operatorname{dim} \mathbf{E}_{\mathbf{c}^{\prime \prime}}+2 a^{\prime} a^{\prime \prime} \sum_{i \rightarrow j} p_{i}^{k} p_{j}^{k}
$$

We substitute $\operatorname{dim} \mathbf{E}_{\mathbf{c}^{\prime}}=a^{\prime 2} \sum_{i \rightarrow j} p_{i}^{k} p_{j}^{k}, \operatorname{dim} \mathbf{E}_{\mathbf{c}^{\prime \prime}}=a^{\prime \prime 2} \sum_{i \rightarrow j} p_{i}^{k} p_{j}^{k}$; we obtain

$$
\operatorname{dim} \mathscr{O}_{\mathbf{c}}=\left(a^{\prime}+a^{\prime \prime}\right)^{2} \sum_{i \rightarrow j} p_{i}^{k} p_{j}^{k}=\operatorname{dim} \mathbf{E}_{\mathbf{c}},
$$

as desired. Lemma 6.7 follows; at the same time, Proposition 6.6 is proved.

Corollary 6.9. In the setup of 6.5 , the codimension of the orbit $\mathscr{O}_{c}$ in the vector space $\mathbf{E}_{\mathbf{d}}$ is given by

$$
-\sum_{h<k ; i} d_{i}^{h} d_{i}^{k}+\sum_{h<k ; i \rightarrow j} d_{j}^{h} d_{i}^{k}
$$


We must show that the sum of the expression (a) with the right-hand side of the expression 6.6(a) is equal to $\operatorname{dim} \mathbf{E}_{\mathrm{d}}$. This sum is

$$
\begin{aligned}
\sum_{k ; i \rightarrow j} d_{i}^{k} d_{j}^{k}+\sum_{h<k ; i \rightarrow j} d_{i}^{h} d_{j}^{k}+\sum_{h<k ; i \rightarrow j} d_{j}^{h} d_{i}^{k} & =\sum_{i \rightarrow j}\left(\sum_{k} d_{i}^{k}\right)\left(\sum_{k} d_{j}^{k}\right) \\
& =\sum_{i \rightarrow j} d_{i} d_{j}=\operatorname{dim} \mathbf{E}_{\mathbf{d}} .
\end{aligned}
$$

The proposition is proved.

6.10. In the setup of 6.5 , assume that $\mathbf{c}_{k}^{\prime} \in \mathbf{N}^{\nu}(k \in[1, \nu])$ satisfy $\operatorname{dim} \mathbf{V}_{\mathbf{c}_{k}^{\prime}}=$ $\operatorname{dim} \mathbf{V}_{\mathbf{c}_{k}}$ for all $k$ and $\mathbf{c}_{k}^{\prime} \neq \mathbf{c}_{k}$ for some $k$. Let $\mathbf{c}^{\prime} \in \mathbf{N}^{\nu}$ be such that

$$
\left(\mathscr{O}_{\mathbf{c}_{1}^{\prime}}, \mathscr{O}_{\mathbf{c}_{2}^{\prime}}, \ldots, \mathscr{O}_{\mathbf{c}_{\nu}^{\prime}}\right) \Longrightarrow \mathscr{O}_{\mathbf{c}^{\prime}}
$$

Using 6.4, we see that

$$
\begin{aligned}
\operatorname{dim}\left(\mathscr{O}_{\mathbf{c}^{\prime}}\right) & \leq \sum_{k=1}^{\nu} \operatorname{dim}\left(\mathscr{O}_{\mathbf{c}_{k}^{\prime}}\right)+\sum_{h<k ; i} d_{i}^{h} d_{i}^{k}+\sum_{h<k ; i \rightarrow j} d_{i}^{h} d_{j}^{k}, \\
\operatorname{dim}\left(\mathscr{O}_{\mathbf{c}}\right) & =\sum_{k=1}^{\nu} \operatorname{dim}\left(\mathscr{O}_{\mathbf{c}_{k}}\right)+\sum_{h<k ; i} d_{i}^{h} d_{i}^{k}+\sum_{h<k ; i \rightarrow j} d_{i}^{h} d_{j}^{k} .
\end{aligned}
$$

Substracting term by term, we obtain

$$
\operatorname{dim}\left(\mathscr{O}_{\mathbf{c}}\right)-\operatorname{dim}\left(\mathscr{O}_{\mathbf{c}^{\prime}}\right) \geq \sum_{k=1}^{\nu}\left(\operatorname{dim}\left(\mathscr{O}_{\mathbf{c}_{k}}\right)-\operatorname{dim}\left(\mathscr{O}_{\mathbf{c}_{k}^{\prime}}\right)\right)
$$

From 6.7 and from our assumption on $\mathbf{c}_{k}^{\prime}$ we deduce that $\operatorname{dim}\left(\mathscr{O}_{\mathbf{c}_{k}}\right)-\operatorname{dim}\left(\mathscr{O}_{\mathbf{c}_{k}^{\prime}}\right) \geq$ 0 for all $k$, with strict inequality for some $k$. This, together with (b) implies

$$
\operatorname{dim}\left(\mathscr{O}_{\mathbf{c}}\right)-\operatorname{dim}\left(\mathscr{O}_{\mathbf{c}^{\prime}}\right)>0
$$

6.11. We now show that the assumption (a) in 6.10 is a consequence of the following assumption:

(a) $E_{\mathbf{i}}^{\mathbf{c}^{\prime}}$ appears with nonzero coefficient in the product $E_{\mathbf{i}}^{\mathbf{c}_{1}^{\prime}} E_{\mathbf{i}}^{\mathbf{c}_{2}^{\prime}} \cdots E_{\mathbf{i}}^{\mathbf{c}_{\nu_{j}^{\prime}}}$ with respect to the basis $B_{\mathrm{i}}$ of $U^{+}$.

From (a) it follows that the statement analogous to (a) is true when $U^{+}$is replaced by $U_{q}^{+}$for large enough $q$. Using the homorphism $\Gamma$ and the identity 5.10 (b), we deduce that

(b) $\mathbf{V}_{\mathbf{c}^{\prime}}$ appears with nonzero coefficient in the product $\mathbf{V}_{\mathbf{c}_{1}^{\prime}} \mathbf{V}_{\mathbf{c}_{2}^{\prime}} \cdots \mathbf{V}_{\mathbf{c}_{\nu}^{\prime}}$ in the algebra $R \Omega$ (notations relative to $F_{q}$.)

Using 6.8(b) repeatedly, we see that (b) implies condition 6.10(a).

\section{A FORMULA FOR $f_{c}$}

7.1. In this section we assume that $F$ is a finite field with $q$ elements, that $\Omega$ is a fixed orientation of the Dynkin graph and that we have fixed $i \in \mathscr{X}$ adapted to $\Omega$. By changing, if necessary, the numbering of the simple roots we may assume that the following condition is satisfied:

(a) if $i \rightarrow j$ is an arrow in $\Omega$ then $j<i$. 
7.2. Let $\mathbf{V}$ be a module of dimension $\mathbf{d}$ in $M \Omega$. For each $i \in[1, n]$, let $\mathbf{V}^{i}$ be the submodule of $\mathbf{V}$ whose $j$-component coincides with the $j$-component of $\mathbf{V}$ for any $j \in[1, i]$ and whose other components are zero; let $\mathbf{V}_{i}$ be the module in $M \Omega$ whose $i$-component is the same as the $i$-component of $\mathbf{V}$ and whose other components are zero. (The maps between the components of $\mathbf{V}_{i}$ are necessarily zero.) Thus we have a canonical exact sequence

$$
0 \longrightarrow \mathbf{v}^{i-1} \longrightarrow \mathbf{v}^{i} \longrightarrow \mathbf{v}_{i} \longrightarrow 0
$$

(where we set $V^{0}=0$ ) and clearly, $\mathbf{V}^{i}$ has exactly one submodule (resp. quotient module) isomorphic to $\mathbf{V}^{i-1}$ (resp. $\left.\mathbf{V}_{i}\right)$. We have $\mathbf{V}_{i}=e_{i}^{\left(\left(d_{i}\right)\right)}$ in $R \Omega$.

From the definition of the multiplication in $R \Omega$, it follows that

$$
\mathbf{V}_{n} \cdots \mathbf{V}_{2} \mathbf{V}_{1}=e_{n}^{\left(\left(d_{n}\right)\right)} \cdots e_{2}^{\left(\left(d_{2}\right)\right)} e_{1}^{\left(\left(d_{1}\right)\right)}
$$

is equal to the sum of all modules of dimension d (one in each isomorphism class) each appearing with coefficient one in the sum. In other words, if we set

$$
e(\mathbf{d})=e_{n}^{\left(\left(d_{n}\right)\right)} \cdots e_{2}^{\left(\left(d_{2}\right)\right)} e_{1}^{\left(\left(d_{1}\right)\right)}
$$

then

$$
e(\mathbf{d})=\sum_{\mathbf{c}} \mathbf{V}_{\mathbf{c}}
$$

where c runs over all elements of $\mathbf{N}^{\nu}$ of $\mathbf{i}$-homogeneity $\mathbf{d}$.

7.3. We now consider the homomorphism $\Gamma$ (see 5.8), defined in terms of $\mathbf{z} \in \mathbf{Z}^{n}$ (see 5.5).

Recall that $\Gamma\left(e_{i}\right)=K_{i}^{-z_{i}} E_{i}$ for all $i$. We have $[[N]] !=q^{N(N-1) / 4}[N] !$; hence $\Gamma\left(e_{i}^{((N))}\right)=q^{N(N-1)\left(2 z_{i}-1\right) / 4} K_{i}^{-N z_{i}} E_{i}^{(N)}$. Hence we have

$$
\Gamma(e(\mathbf{d}))=q^{r_{\mathrm{d}} / 2} K(\mathbf{d}) E_{n}^{\left(d_{n}\right)} E_{n-1}^{\left(d_{n-1}\right)} \cdots E_{1}^{\left(d_{1}\right)}
$$

where $K(\mathbf{d})=K_{1}^{-z_{1} d_{1}} \cdots K_{n}^{-z_{n} d_{n}}($ see $5.10(\mathbf{c}))$, and

$$
r_{\mathbf{d}}=\sum_{i} d_{i}\left(d_{i}-1\right)\left(2 z_{i}-1\right) / 2-\sum_{i \rightarrow j} d_{i} d_{j} z_{j} .
$$

We now apply $\Gamma$ to the identity $7.2(a)$. Using $5.10($ b), we obtain an equality of the following form in $U_{q}$ :

$$
q^{r_{\mathbf{d}} / 2} K(\mathbf{d}) E_{n}^{\left(d_{n}\right)} E_{n-1}^{\left(d_{n-1}\right)} \cdots E_{1}^{\left(d_{1}\right)}=\sum_{\mathbf{c}} q^{f_{\mathbf{c}} / 2} K(\mathbf{d}) E_{\mathbf{i}}^{\mathbf{c}}
$$

where c runs over all elements of $\mathbf{N}^{\nu}$ of i-homogeneity $\mathbf{d}$ and the integers $f_{\mathbf{c}}$ are independent of $q$. We can cancel $K(\mathbf{d})$ in the identity (b). Moreover, since (b) holds for all $q$, we must have a corresponding identity in $U$ :

$$
E_{n}^{\left(d_{n}\right)} E_{n-1}^{\left(d_{n-1}\right)} \cdots E_{1}^{\left(d_{1}\right)}=\sum_{\mathbf{c}} v^{f_{\mathbf{c}}-r_{\mathbf{d}}} E_{\mathbf{i}}^{\mathbf{c}}
$$


Lemma 7.4. Exactly one exponent $f_{\mathbf{c}}-r_{\mathbf{d}}$ in the sum $7.3(\mathrm{c})$ is equal to zero.

We associate $\mathbf{i}^{\prime} \in \mathscr{Z}$ to $\mathbf{i}$ as in 2.11. Assume the truth of the following statement:

(a) $E_{n}^{\left(d_{n}\right)} E_{n-1}^{\left(d_{n-1}\right)} \cdots E_{1}^{\left(d_{1}\right)}$ belongs to the basis $B_{\mathrm{i}^{\prime}}$ of $\mathscr{L}$ (see $2.3(\mathrm{~b})$ ).

From 2.3(c) it follows that any element of the basis $B_{\mathrm{i}^{\prime}}$ can be expressed as a linear combination of elements in the basis $B_{\mathbf{i}}$ with coefficients in $\mathbf{Z}\left[v^{-1}\right]$ and that exactly one of these coefficients has constant term 1 . Applying this to the element in (a), we see that the conclusion of the lemma holds.

It remains to prove (a). Using $2.11(a)$, we see that it is enough to verify that

$$
E_{1}^{\left(d_{1}\right)} E_{2}^{\left(d_{2}\right)} \cdots E_{n}^{\left(d_{n}\right)} \in B_{\mathbf{i}}
$$

From 2.8 we see that there exists a sequence $l(1)<l(2)<\cdots<l(n)$ in $[1, \nu]$ and a permutation $p$ of $[1, n]$ such that

$$
\alpha_{p(j)}=s_{i_{1}} s_{i_{2}} \cdots s_{i_{l(j-1)}}\left(\alpha_{i_{l(j)}}\right)
$$

for all $j \in[1, n]$. Using $1.3(\mathrm{c})$ it then follows that

$$
\tilde{T}_{i_{1}} \tilde{T}_{i_{2}} \cdots \tilde{T}_{i_{l(j-1)}}\left(E_{i_{l(j)}}\right)=E_{p(j)}
$$

for all $j \in[1, n]$ and from the definition of $B_{\mathrm{i}}$ we see that

$$
E_{p(1)}^{\left(d_{p(1)}\right)} E_{p(2)}^{\left(d_{p(2)}\right)} \cdots E_{p(n)}^{\left(d_{p(n)}\right)} \in B_{\mathrm{i}} .
$$

From 4.14(b) we see that

(e) if $i \rightarrow j$ is an arrow in $\Omega$, then $p^{-1}(j)<p^{-1}(i)$.

Since $E_{i} E_{j}=E_{j} E_{i}$ if $i \neq j$ are not joined in the Dynkin graph, we see from (e) and 7.1(a) that the factors in (d) can be rearranged so that the element in (d) is equal to that in (b) and thus (b) holds. The lemma is proved.

Lemma 7.5. For any $\mathbf{c} \in \mathbf{N}^{\nu}$ of $\mathbf{i}$-homogeneity $\mathbf{d}$ we have

$$
f_{\mathbf{c}}-r_{\mathbf{d}}=-\delta(\mathbf{c})
$$

where

$$
\delta(\mathbf{c})=-\sum_{h<k ; i} d_{i}^{h} d_{i}^{k}+\sum_{h<k ; i \rightarrow j} d_{j}^{h} d_{i}^{k} .
$$

It is enough to prove (a) for a (c, d) under the following inductive assumption: (a) holds when $(\mathbf{c}, \mathbf{d})$ is replaced by $\left(\mathbf{c}^{\prime}, \mathbf{d}^{\prime}\right)$ such that at least one of the conditions (b),(c) below is satisfied.

(b) Any coordinate of $\mathbf{d}^{\prime}$ is $\leq$ than the corresponding coordinate of $\mathbf{d}$ and $\mathbf{d}^{\prime} \neq \mathbf{d}$.

(c) We have $\mathbf{d}^{\prime}=\mathbf{d}$ and the dimension of the orbit $\mathscr{O}_{\mathbf{c}^{\prime}}$ is strictly smaller than that of $\mathscr{O}_{c}$. 
Let $\mathbf{c}_{k} \in \mathbf{N}^{\nu}$ be as in 5.11. Let $\mathbf{d}^{k}=\left(d_{1}^{k}, \ldots, d_{n}^{k}\right)$ be the i-homogeneity of $\mathrm{c}_{k}$. We can rewrite $5.11(\mathrm{~b})$ as follows:

$$
f_{\mathbf{c}}=\sum_{k} f_{\mathbf{c}_{k}}-\sum_{k^{\prime}<k ; j \rightarrow j^{\prime}} d_{j}^{k} d_{j^{\prime}}^{k^{\prime}} z_{j}-\sum_{k^{\prime}<k ; j \rightarrow j^{\prime}} d_{j}^{k^{\prime}} d_{j^{\prime}}^{k} z_{j^{\prime}}+2 \sum_{k^{\prime}<k ; i} d_{i}^{k} d_{i}^{k^{\prime}} z_{i} .
$$

From $7.3(\mathrm{a})$ we have

$$
\begin{aligned}
r_{\mathbf{d}}-\sum_{k} r_{\mathbf{d}^{k}}= & \sum_{i}\left(\begin{array}{c}
d_{i} \\
2
\end{array}\right)\left(2 z_{i}-1\right)-\sum_{k, i}\left(\begin{array}{c}
d_{i}^{k} \\
2
\end{array}\right)\left(2 z_{i}-1\right) \\
& -\sum_{i \rightarrow j} d_{i} d_{j} z_{j}+\sum_{k ; i \rightarrow j} d_{i}^{k} d_{j}^{k} z_{j} \\
= & \sum_{k^{\prime}<k ; i} d_{i}^{k^{\prime}} d_{i}^{k}\left(2 z_{i}-1\right)-\sum_{k^{\prime}<k ; i \rightarrow j}\left(d_{i}^{k^{\prime}} d_{j}^{k}+d_{i}^{k} d_{j}^{k^{\prime}}\right) z_{j} .
\end{aligned}
$$

It follows that

$$
\begin{aligned}
f_{\mathbf{c}}-r_{\mathbf{d}}-\sum_{k}\left(f_{\mathbf{c}_{k}}-r_{\mathbf{d}^{k}}\right) & =-\sum_{k^{\prime}<k ; j \rightarrow j^{\prime}} d_{j}^{k} d_{j^{\prime}}^{k^{\prime}}\left(z_{j}-z_{j^{\prime}}\right)+\sum_{k^{\prime}<k ; i} d_{i}^{k^{\prime}} d_{i}^{k} \\
& =-\sum_{k^{\prime}<k ; j \rightarrow j^{\prime}} d_{j}^{k} d_{j^{\prime}}^{k^{\prime}}+\sum_{k^{\prime}<k ; i} d_{i}^{k^{\prime}} d_{i}^{k} \\
& =-\delta(\mathbf{c})=-\delta(\mathbf{c})+\sum_{k} \delta\left(\mathbf{c}_{k}\right) .
\end{aligned}
$$

(We have used the equality $\delta\left(c_{k}\right)=0$.) Now (d) shows that if (a) holds for each $c_{k}$ then it also holds for $\mathbf{c}$. Using the induction hypothesis, we see that we may assume that $\mathbf{c}=\mathbf{c}_{k}$ for some $k$. In this case, the orbit $\mathscr{O}_{c}$ is open (by 6.7). Hence for any $c^{\prime} \neq c$ with the same $i$-homogeneity as $c$, we have

$$
\operatorname{dim} \mathscr{O}_{\mathbf{c}^{\prime}}<\operatorname{dim} \mathscr{O}_{\mathbf{c}}
$$

and the induction hypothesis applies to $c^{\prime}$ and gives

$$
f_{\mathbf{c}^{\prime}}-r_{\mathbf{d}}=-\delta\left(\mathbf{c}^{\prime}\right)=-\operatorname{codim} \mathscr{\Theta}_{\mathbf{c}^{\prime}}<0
$$

(We have used 6.9 and (e).) Hence in 7.3(c), all exponents of $v$ (except possibly for the single term corresponding to our fixed c) are $<0$. Since in our case $\delta(\mathbf{c})=0$, we must only prove that $f_{\mathbf{c}}-r_{\mathbf{d}}=0$. Assume that this is not so. Then, in 7.3(c), all exponents of $v$, without exception, are different from 0 . This contradicts 7.4. The lemma is proved.

7.6. We can restate 7.3 (c) taking into account 7.5 in the following result.

Proposition 7.7. We have

$$
E_{n}^{\left(d_{n}\right)} E_{n-1}^{\left(d_{n-1}\right)} \cdots E_{1}^{\left(d_{1}\right)}=\sum_{\mathbf{c}} v^{-\delta(\mathbf{c})} E_{\mathbf{i}}^{\mathbf{c}}
$$


where the sum is taken over all $\mathbf{c} \in \mathbf{N}^{\nu}$ of $\mathbf{i}$-homogeneity $\mathbf{d}$ and $\delta(\mathbf{c})$ is the codimension of the orbit $\mathscr{O}_{\mathbf{c}}$.

7.8. As before,we associate $\mathbf{c}_{k}, \mathbf{d}^{k}(k \in[1, \nu])$ to $\mathbf{c}, \mathbf{d}$. Let

$$
E((\mathbf{c}))=E\left(\mathbf{d}^{1}\right) E\left(\mathbf{d}^{2}\right) \cdots E\left(\mathbf{d}^{\nu}\right)
$$

where

$$
E\left(\mathbf{d}^{k}\right)=E_{n}^{\left(d_{n}^{k}\right)} E_{n-1}^{\left(d_{n-1}^{k}\right)} \cdots E_{1}^{\left(d_{1}^{k}\right)} .
$$

Write

$$
E((\mathbf{c}))=\sum_{\mathbf{c}^{\prime}} h_{\mathbf{c}^{\prime}}^{\mathbf{c}} E_{\mathbf{i}}^{\mathbf{c}^{\prime}}
$$

where $\mathbf{c}^{\prime}$ runs over the set of elements in $\mathbf{N}^{\nu}$ of i-homogeneity $\mathbf{d}$ and $h_{\mathbf{c}^{\prime}}^{\mathbf{c}} \in A$. From 7.7 and the results in $6.10,6.11$, we see that

$$
h_{\mathrm{c}}^{\mathrm{c}}=1
$$

(since $\delta\left(\mathbf{c}_{k}\right)=0$ for all $k$, by 6.7$)$ and

$$
h_{\mathbf{c}^{\prime}}^{\mathbf{c}} \neq 0, \mathbf{c} \neq \mathbf{c}^{\prime} \Longrightarrow \operatorname{dim}\left(\mathscr{O}_{\mathbf{c}^{\prime}}\right)<\operatorname{dim}\left(\mathscr{O}_{\mathbf{c}}\right) \text {. }
$$

It is clear that

$$
\overline{E((\mathbf{c}))}=E((\mathbf{c}))
$$

We can write

$$
\overline{E_{\mathrm{i}}^{\mathbf{c}}}=\sum_{\mathbf{c}^{\prime}} \omega_{\mathbf{c}^{\prime}}^{\mathbf{c}} E_{\mathbf{i}}^{\mathbf{c}^{\prime}}
$$

where $\mathbf{c}^{\prime}$ runs over the set of elements in $\mathbf{N}^{\nu}$ of $\mathbf{i}$-homogeneity $\mathbf{d}$ and $\omega_{\mathbf{c}^{\prime}}^{\mathbf{c}} \in A$ are uniquely determined.

Proposition 7.9. We have $\omega_{\mathbf{c}}^{\mathbf{c}}=1 ;$ moreover, $\omega_{\mathbf{c}^{\prime}}^{\mathbf{c}} \neq 0, \mathbf{c} \neq \mathbf{c}^{\prime}$ implies $\operatorname{dim}\left(\mathscr{O}_{\mathbf{c}^{\prime}}\right)<$ $\operatorname{dim}\left(\mathscr{O}_{\mathbf{c}}\right)$.

Using $7.8(\mathrm{e}),(\mathrm{f})$, we see that

$$
\sum_{\mathbf{c}^{\prime}} \overline{h_{\mathbf{c}^{\prime}}^{\mathbf{c}}} \sum_{\mathbf{c}^{\prime \prime}} \omega_{\mathbf{c}^{\prime \prime}}^{\mathbf{c}^{\prime}} E_{\mathbf{i}}^{c^{\mathbf{c}^{\prime \prime}}}=\sum_{\mathbf{c}^{\prime \prime}} h_{\mathbf{c}^{\prime \prime}}^{\mathbf{c}} E_{\mathbf{i}}^{\mathbf{c}^{\mathbf{c}^{\prime \prime}} ;}
$$

hence

$$
h_{\mathbf{c}^{\prime \prime}}^{\mathbf{c}}=\sum_{\mathbf{c}^{\prime}} \overline{h_{\mathbf{c}^{\prime}}^{\mathbf{c}}} \omega_{\mathbf{c}^{\prime \prime}}^{\mathbf{c}^{\prime}}
$$

for all $\mathbf{c}, \mathbf{c}^{\prime \prime}$ of i-homogeneity $\mathbf{d}$. For such $\mathbf{c}, \mathbf{c}^{\prime \prime}$ we say that $\mathbf{c}^{\prime \prime} \leq \mathbf{c}$ if either $\mathbf{c}=\mathbf{c}^{\prime \prime}$ or if $\mathbf{c} \neq \mathbf{c}^{\prime \prime}$ and $\operatorname{dim}\left(\mathscr{O}_{\mathbf{c}^{\prime}}\right)<\operatorname{dim}\left(\mathscr{O}_{\mathbf{c}}\right)$. From 7.8(c),(d) we see that the matrix $\left(h_{\mathbf{c}^{\prime \prime}}^{\mathbf{c}}\right)$ is upper triangular (with respect to $\leq$ ) with diagonal entries equal to 1 . From (a) it then follows that the same must hold for the matrix $\left(\omega_{\mathbf{c}^{\prime \prime}}^{\mathbf{c}}\right)$. The proposition is proved. 
7.10. In this and the following subsection we shall give the proof of Theorem 3.2. Let $I$ be a finite partially ordered set and assume that for any $i^{\prime}, i$ in $I$ we are given elements $u_{i^{\prime}}^{i} \in A$ such that

$$
\sum_{i^{\prime \prime}} u_{i^{\prime}}^{i^{\prime \prime}} \overline{u_{i^{\prime \prime}}^{i}}=\delta_{i, i^{\prime \prime}}
$$

for all $i^{\prime}, i \in I$,

$$
u_{i}^{i}=1 \text { for all } i \in I
$$

and

$$
u_{i}^{i^{\prime}}=0 \text { unless } i^{\prime} \leq i
$$

Then the system of equations

$$
Z_{i^{\prime}}^{i}=\sum_{i^{\prime \prime}: i^{\prime} \leq i^{\prime \prime} \leq i} u_{i^{\prime}}^{i^{\prime \prime}} \overline{Z_{i^{\prime \prime}}^{i}}
$$

$\forall i^{\prime} \leq i$ with unknowns $Z_{i^{\prime}}^{i} \in \mathbf{Z}\left[v^{-1}\right]\left(i^{\prime} \leq i\right)$ has a unique solution such that $Z_{i}^{i}=1$ for all $i \in I$ and $Z_{i^{\prime}}^{i} \in v^{-1} \mathbf{Z}\left[v^{-1}\right]$ for $i^{\prime}<i$. This can be proved exactly as in [L3, p. 101].

7.11. We apply this in the case where $I=\chi_{\mathbf{i}}^{-1}(\mathbf{d})$ (see 2.8) for some $\mathbf{d} \in \mathbf{N}^{n}$. We regard $I$ as a partially ordered set with the partial order $\mathbf{c}^{\prime} \leq \mathbf{c}$ defined in the proof of 7.9. We take $u_{i^{\prime}}^{i}$ in 7.10 to be $\omega_{\mathbf{c}^{\prime}}^{\mathbf{c}}$. This satisfies 7.10 (a) since - : $U^{+} \rightarrow U^{+}$is an involution. It satisfies $7.10(\mathrm{~b}),(\mathrm{c})$, by 7.9 . We see that one can solve uniquely the system of equations

$$
\zeta_{\mathbf{c}^{\prime}}^{\mathbf{c}}=\sum_{\mathbf{c}^{\prime \prime}: \mathbf{c}^{\prime} \leq \mathbf{c}^{\prime \prime} \leq \mathbf{c}} \omega_{\mathbf{c}^{\prime}}^{\mathbf{c}^{\prime \prime} \overline{\bar{c}_{\mathbf{c}^{\prime \prime}}^{\mathbf{c}}}}
$$

with unknowns $\zeta_{\mathbf{c}^{\prime}}^{\mathbf{c}} \in \mathbf{Z}\left[v^{-1}\right], \mathbf{c}^{\prime} \leq \mathbf{c}$ in $I$ so that $\zeta_{\mathbf{c}}^{\mathbf{c}}=1$ for all $\mathbf{c} \in I$ and $\zeta_{\mathbf{c}^{\prime}}^{\mathbf{c}} \in v^{-1} \mathbf{Z}\left[v^{-1}\right]$ for all $\mathbf{c}^{\prime}<\mathbf{c}$. For $\mathbf{c} \in I$, let

$$
\mathscr{E}^{\mathbf{c}}=\sum_{\mathbf{c}^{\prime}} \zeta_{\mathbf{c}^{\prime}}^{\mathbf{c}} E_{\mathbf{i}}^{\mathbf{c}^{\prime}}
$$

where, in the sum we take $\mathbf{c}^{\prime} \in I, \mathbf{c}^{\prime} \leq \mathbf{c}$. Let $J$ be the family consisting of the elements $\mathscr{E}^{\mathbf{c}}$ where $\mathbf{c} \in \chi_{\mathbf{i}}^{-1}(\mathbf{d})$ for various $\mathbf{d} \in \mathbf{N}^{n}$. From (a) it follows that

$$
\text { each element of } J \text { is fixed by }{ }^{-} \text {. }
$$

Moreover, from (b) and the definition we see that $J$ is a $\mathbf{Z}\left[v^{-1}\right]$-basis of $\mathscr{L}$ and that $\pi\left(\mathscr{E}^{\mathbf{c}}\right)=\pi\left(E_{\mathbf{i}}^{\mathbf{c}}\right)$ so that $\pi$ applies $J$ bijectively onto $B$. Using (c) we see that $\mathscr{L} \cap \overline{\mathscr{L}}$ is precisely the free $\mathrm{Z}$-module with basis $J$, and that 3.2(a) holds with $\mathbf{B}=J$. The other statements of 3.2 also follow. Thus Theorem 3.2 is proved.

7.12. From 7.11(b) we see that $\mathbf{B}=\sqcup_{\mathbf{d}}\left(\mathbf{B} \cap \mathbf{U}_{\mathbf{d}}^{+}\right)$. 
7.13. We now define in $R \Omega$

$$
e((\mathbf{c}))=e\left(\mathbf{d}^{1}\right) e\left(\mathbf{d}^{2}\right) \cdots e\left(\mathbf{d}^{\nu}\right)
$$

where $e\left(\mathbf{d}^{k}\right)$ is as in 7.2. We have

$$
e((\mathbf{c}))=\sum_{\mathbf{c}^{\prime}} \tilde{h}_{\mathbf{c}^{\prime}}^{\mathbf{c}} \mathbf{v}_{\mathbf{c}^{\prime}}
$$

where $\tilde{h}_{\mathbf{c}^{\prime}}^{\mathrm{c}} \in \mathbf{C}$. From 5.12 it follows that, as a function of $q$,

(c) $\tilde{h}_{\mathbf{c}^{\prime}}^{\mathbf{c}}$ is a polynomial in $q$ with integer coefficients.

With the notation of 7.3 we have

$$
\begin{aligned}
\Gamma(e((\mathbf{c}))) & =\Gamma\left(e\left(\mathbf{d}^{1}\right)\right) \Gamma\left(e\left(\mathbf{d}^{2}\right)\right) \cdots \Gamma\left(e\left(\mathbf{d}^{\nu}\right)\right) \\
& =q^{r_{\mathbf{d}_{1}} / 2} K\left(\mathbf{d}^{1}\right) E\left(\mathbf{d}^{1}\right) \cdots q^{r_{\mathbf{d}_{\nu}} / 2} K\left(\mathbf{d}^{\nu}\right) E\left(\mathbf{d}^{\nu}\right) \\
& =q^{r_{\mathrm{d}} / 2+m / 2} K(\mathbf{d}) E((\mathbf{c}))
\end{aligned}
$$

where

$$
\begin{aligned}
m= & -r_{\mathbf{d}}+\sum_{k} r_{\mathbf{d}_{k} / 2}+\sum_{k^{\prime}<k ; i, j} d_{i}^{k^{\prime}} d_{j}^{k} z_{j} a_{i j} \\
= & -\sum_{k^{\prime}<k ; i} d_{i}^{k^{\prime}} d_{i}^{k}\left(2 z_{i}-1\right)+\sum_{k^{\prime}<k ; i \rightarrow j}\left(d_{i}^{k^{\prime}} d_{j}^{k}+d_{i}^{k} d_{j}^{k^{\prime}}\right) z_{j} \\
& +2 \sum_{k^{\prime}<k ; i} d_{i}^{k^{\prime}} d_{i}^{k} z_{i}-\sum_{k^{\prime}<k ; i \rightarrow j} d_{i}^{k^{\prime}} d_{j}^{k} z_{j}-\sum_{k^{\prime}<k ; i \rightarrow j} d_{j}^{k^{\prime}} d_{i}^{k} z_{i} \\
= & -\sum_{k^{\prime}<k ; i \rightarrow j} d_{j}^{k^{\prime}} d_{i}^{k}+\sum_{k^{\prime}<k ; i} d_{i}^{k^{\prime}} d_{i}^{k} \\
= & -\delta(\mathbf{c}) .
\end{aligned}
$$

Thus

$$
\Gamma(e((\mathbf{c})))=q^{\left(r_{\mathbf{d}}-\delta(\mathbf{c})\right) / 2} K(\mathbf{d}) E((\mathbf{c})) .
$$

On the other hand, we can rewrite $5.10(\mathrm{~b})$ taking into account 7.5:

$$
\Gamma\left(\mathbf{V}_{\mathbf{c}}\right)=q^{\left(-\delta_{\mathbf{c}}+r_{\mathbf{d}}\right) / 2} K(\mathbf{d}) E_{\mathbf{i}}^{\mathbf{c}}
$$

Applying $\Gamma$ to (b) and using (d),(e), we obtain

$$
q^{\left(r_{\mathbf{d}}-\delta(\mathbf{c})\right) / 2} K(\mathbf{d}) E((\mathbf{c}))=\sum_{\mathbf{c}^{\prime}} \tilde{h}_{\mathbf{c}^{\prime}}^{\mathbf{c}} q^{\left(-\delta\left(\mathbf{c}^{\prime}\right)+r_{\mathbf{d}}\right) / 2} K(\mathbf{d}) E_{\mathbf{i}}^{\mathbf{c}^{\prime}}
$$

Comparing this with $7.8(\mathrm{~b})$, we obtain

$$
h_{\mathbf{c}^{\prime}}^{\mathbf{c}}=q^{\left(\delta(\mathbf{c})-\delta\left(\mathbf{c}^{\prime}\right)\right) / 2} \tilde{h}_{\mathbf{c}^{\prime}}^{\tilde{\mathbf{c}}}
$$

The left-hand side is an element of $A$ evaluated at $v=q^{1 / 2}$, while to the right side we can apply (c). Since (f) holds for all $q$, we deduce that

(g) $v^{-\delta(\mathbf{c})+\delta\left(\mathbf{c}^{\prime}\right)} h_{\mathbf{c}^{\prime}}^{\mathbf{c}} \in A$ is a polynomial in $v^{2}$ with integral coefficients. 
Proposition 7.14. Let

$$
\omega_{\mathbf{c}^{\prime}}^{\prime \mathbf{c}}=v^{-\delta(\mathbf{c})+\delta\left(\mathbf{c}^{\prime}\right)} \omega_{\mathbf{c}^{\prime}}^{\mathbf{c}} \in A
$$

Then $\omega_{\mathbf{c}^{\prime}}^{\prime c}$ is contained in $\mathbf{Z}\left[v^{2}, v^{-2}\right]$.

We set

$$
h_{\mathbf{c}^{\prime}}^{\prime \mathbf{c}}=v^{-\delta(\mathbf{c})+\delta\left(\mathbf{c}^{\prime}\right)} h_{\mathbf{c}^{\prime}}^{\mathbf{c}} \in A
$$

From 7.9(a) we deduce

$$
h_{\mathbf{c}^{\prime \prime}}^{\prime \mathbf{c}}=\sum_{\mathbf{c}^{\prime}} v^{-2 \delta(\mathbf{c})+2 \delta\left(\mathbf{c}^{\prime}\right)} \overline{h_{\mathbf{c}^{\prime}}^{\prime \mathbf{c}}} \omega_{\mathbf{c}^{\prime \prime}}^{\prime \mathbf{c}^{\prime}}
$$

By $7.13(\mathrm{~g})$, the matrix $\left(h_{\mathbf{c}^{\prime}}^{\prime \mathbf{c}}\right)$ and the matrix $\left(v^{-2 \delta(\mathbf{c})+2 \delta\left(\mathbf{c}^{\prime}\right)} \overline{h_{\mathbf{c}^{\prime}}^{\prime \mathbf{c}}}\right)$ have entries in $\mathbf{Z}\left[v^{2}, v^{-2}\right]$; moreover, these matrices are triangular with 1 on diagonal $(7.8(\mathrm{c}),(\mathrm{d}))$. Hence, by $(\mathrm{a})$, the matrix $\left(\omega_{\mathbf{c}^{\prime}}^{\prime \mathbf{c}}\right)$ must have the same properties. The proposition follows.

\section{Finite-dimensional U-Modules}

8.1. Let $\mathbf{d}=\left(d_{1}, \ldots, d_{n}\right) \in \mathbf{N}^{n}$ and let $I_{\mathbf{d}}$ (resp. $J_{\mathbf{d}}$ ) be the left (resp. right) ideal in $\mathbf{U}^{+}$generated by the elements $E_{1}^{d_{1}+1}, \ldots, E_{n}^{d_{n}+1}$.

Theorem 8.2. (a) The intersection $\mathbf{B} \cap I_{\mathbf{d}}$ is an $A^{\prime}$-basis of $I_{\mathbf{d}}$.

(b) The intersection $\mathbf{B} \cap J_{\mathbf{d}}$ is an $A^{\prime}$-basis of $J_{\mathbf{d}}$.

The proof will be given in 8.6.

8.3. We may assume that the numbering of the simple roots is such that for some $t \in[1, n]$, we have $a_{i j}=0$ whenever $i \neq j \in[1, n]$ are both $\leq t$ or both $>t$. We define a sequence

$$
\mathbf{i}=\left(i_{1}, i_{2}, \ldots, i_{\nu}\right)
$$

by the requirement that $i_{r}=r$ if $1 \leq r \leq n, i_{r^{\prime}}=i_{r^{\prime \prime}}$ if $r^{\prime} \equiv r^{\prime \prime}(\bmod n)$. It is known [B, Ex.2, $\S 6, \mathrm{Ch} . \mathrm{V}]$ that $\mathbf{i} \in \mathscr{X}$. Although we do not need this here, we note that $\mathbf{i}$ is adapted to the unique orientation for which $\{1,2, \ldots, t\}$ are sinks and $\{t+1, t+2, \ldots, n\}$ are sources. Let $J_{\mathbf{d}}^{\prime}$ (resp. $J_{\mathbf{d}}^{\prime \prime}$ ) be the right ideal of $\mathbf{U}^{+}$generated by the elements $E_{1}^{d_{1}+1}, \ldots, E_{t}^{d_{t}+1}$ (resp. $E_{t+1}^{d_{t+1}+1}, \ldots, E_{n}^{d_{n}+1}$ ). It is clear that the intersection $B_{\mathrm{i}} \cap J_{\mathrm{d}}^{\prime}$ is an $A^{\prime}$-basis of $J_{\mathrm{d}}^{\prime}$; this intersection consists of all elements $E_{\mathbf{i}}^{\mathbf{c}}$ such that $\mathbf{c}=\left(c_{1}, \ldots, c_{\nu}\right)$ satisfies

$$
c_{i} \geq d_{i}+1 \text { for } 1 \leq i \leq t \text {. }
$$

Similarly, if $\mathbf{j}=\left(j_{1}, j_{2}, \ldots, j_{\nu}\right)$ is the sequence defined by $j_{r}=n+1-r$ if $1 \leq r \leq n, j_{r^{\prime}}=j_{r^{\prime \prime}}$ if $r^{\prime} \equiv r^{\prime \prime}(\bmod n)$ then $\mathbf{j} \in \mathscr{X}$ and the intersection $B_{\mathbf{j}} \cap J_{\mathbf{d}}^{\prime \prime}$ is an $A^{\prime}$-basis of $J_{\mathbf{d}}^{\prime \prime}$; this intersection consists of all elements $E_{\mathbf{j}}^{c^{\prime}}$ such that $\mathbf{c}^{\prime}=\left(c_{1}^{\prime}, \ldots, c_{\nu}^{\prime}\right)$ satisfies

$$
c_{i}^{\prime} \geq d_{n-i+1}+1 \text { for } 1 \leq i \leq n-t .
$$


Hence

(c) the bijection $\mathbf{N}^{\nu} \cong B_{\mathbf{i}}$ given by $\mathbf{c} \rightarrow E_{\mathbf{i}}^{\mathbf{c}}$ restricts to a bijection

$$
\left\{\mathbf{c} \in \mathbf{N}^{\nu} \mid c_{i} \leq d_{i}(1 \leq i \leq t)\right\} \cong B_{\mathbf{i}}-\left(B_{\mathbf{i}} \cap J_{\mathbf{d}}^{\prime}\right) \text {. }
$$

and

(d) the bijection $\mathbf{N}^{\nu} \cong B_{\mathbf{j}}$ given by $\mathbf{c} \rightarrow E_{\mathbf{j}}^{\mathbf{c}}$ restricts to a bijection

$$
\left\{\mathbf{c} \in \mathbf{N}^{\nu} \mid c_{i}^{\prime} \leq d_{n-i+1}(1 \leq i \leq n-t)\right\} \cong B_{\mathbf{j}}-\left(B_{\mathbf{j}} \cap J_{\mathbf{d}}^{\prime \prime}\right) .
$$

8.4. Under the canonical bijections

$$
\mathbf{B} \cong B_{\mathbf{i}}, \quad \mathbf{B} \cong B_{\mathbf{j}}
$$

(the compositions of $\mathbf{B} \cong B \cong B_{\mathbf{i}}$ (resp. $\mathbf{B} \cong B \cong B_{\mathbf{j}}$ ), see $3.2(\mathrm{a})$ ) the subset $B_{\mathbf{i}} \cap J_{\mathbf{d}}^{\prime}$ of $B_{\mathbf{i}}$ corresponds to a subset $\mathbf{B}^{\prime}(\mathbf{d})$ of $\mathbf{B}$ and the subset $B_{\mathbf{j}} \cap J_{\mathbf{d}}^{\prime \prime}$ corresponds to a subset $\mathbf{B}^{\prime \prime}(\mathbf{d})$ of $\mathbf{B}$.

Lemma 8.5. (a) $\mathbf{B}^{\prime}(\mathbf{d})$ is an $A^{\prime}$-basis of $J_{\mathbf{d}}^{\prime}$.

(b) $\mathbf{B}^{\prime \prime}$ (d) is an $A^{\prime}$-basis of $J_{\mathbf{d}}^{\prime \prime}$.

We shall only prove (a); the proof of (b) is the same (by symmetry). It is clear from the definition that $J_{\mathbf{d}}^{\prime \prime}$ is stable under the involution ${ }^{-}: \mathbf{U}^{+} \rightarrow \mathbf{U}^{+}$. Hence the elements $\omega_{\mathbf{c}^{\prime}}^{\mathbf{c}} \in A$ (see 7.8) have the following property:

(c) if $\mathrm{c}$ satisfies $8.3(\mathrm{a})$ and $\omega_{\mathbf{c}^{\prime}}^{\mathrm{c}} \neq 0$ then $\mathbf{c}^{\prime}$ satisfies $8.3(\mathrm{a})$.

From the system of equations 7.11 (a) we see, using (c), that

(d) if c satisfies 8.3(a) and $\zeta_{\mathbf{c}^{\prime}}^{c} \neq 0$ (see 7.11) then $\mathbf{c}^{\prime}$ satisfies 8.3(a).

Hence, if c satisfies 8.3(a), then the element $\mathscr{E}^{c}$ of $\mathbf{B}^{\prime}$ (d) (defined in 7.11(b)) is a linear combination of elements in $B_{\mathbf{i}} \cap J_{\mathbf{d}}^{\prime}$; hence, $\mathbf{B}^{\prime}(\mathbf{d})$ is contained in $J_{\mathbf{d}}^{\prime}$. This linear combination is given by a "unipotent" matrix; hence any element of $B_{\mathbf{i}} \cap J_{\mathbf{d}}^{\prime}$ is a linear combination of elements in $\mathbf{B}^{\prime}(\mathbf{d})$. Since $B_{\mathbf{i}} \cap J_{\mathbf{d}}^{\prime}$ is an $A^{\prime}$-basis of $J_{\mathbf{d}}^{\prime}$, it follows that $\mathbf{B}^{\prime}(\mathbf{d})$ is an $A^{\prime}$-basis of $J_{\mathbf{d}}^{\prime}$. The lemma is proved.

8.6. It is clear that $J_{\mathbf{d}}=J_{\mathbf{d}}^{\prime}+J_{\mathbf{d}}^{\prime \prime}$. Hence from 8.5 it follows that the union $\mathbf{B}^{\prime}(\mathbf{d}) \cup \mathbf{B}^{\prime \prime}(\mathbf{d})$ generates $J_{\mathbf{d}}$ as an $A^{\prime}$-vector space. Since this union is part of the basis $\mathbf{B}$ of $\mathbf{U}^{+}, 8.2$ (b) follows. It is clear that $\Psi\left(J_{\mathbf{d}}\right)=I_{\mathbf{d}}$ ( $\Psi$ as in 1.4). Since $\Psi(\mathbf{B})=\mathbf{B}$, we see that $8.2(\mathrm{a})$ is a consequence of $8.2(\mathrm{~b})$.

8.7. It is known that there is a simple $\mathrm{U}$-module $L_{\mathbf{d}}$ of finite dimension over $A^{\prime}$ with a nonzero vector $x_{0}$ such that $F_{i} x_{0}=0, K_{i} x_{0}=v^{-d_{i}} x_{0}$ for all $i$; moreover $\left(L_{\mathbf{d}}, x_{0}\right)$ is unique up to a unique isomorphism.

Lemma 8.8. There is a unique isomorphism of $\mathbf{Q}$-vector spaces ${ }^{-}: L_{\mathbf{d}} \cong L_{\mathbf{d}}$ such that $\overline{u x}=\bar{u} \bar{x}$ for all $u \in \mathbf{U}, x \in L_{\mathbf{d}}$ and $\overline{x_{0}}=x_{0}$. Its square is equal to 1 .

We can consider a new $\mathbf{U}$-module structure on $L_{\mathbf{d}}: u \in \mathbf{U}$ acts in the new module structure as $\bar{u}$ in the original module structure. In particular, multiplication by $v$ in the new module structure is multiplication by $v^{-1}$ in the original module structure. It is clear that the new module satisfies the defining property of the original module; we use the uniqueness statement in 8.7 and the lemma follows. 
8.9. Let $L_{\mathbf{d}, A}=U^{+} x_{0}=U x_{0} \subset L_{\mathbf{d}}$. Let $\mathscr{L}_{\mathbf{d}}$ be the $\mathbf{Z}\left[v^{-1}\right]$-submodule $\mathscr{L} x_{0}$ of $L_{\mathbf{d}}$. Let $\tau: \mathscr{L}_{\mathbf{d}} \rightarrow \mathscr{L}_{\mathbf{d}} / v^{-1} \mathscr{L}_{\mathbf{d}}$ be the canonical projection. From 2.3 we see that

(a) for any fixed $\mathbf{i} \in \mathscr{X}$, the elements $u\left(x_{0}\right) \quad\left(u \in B_{\mathbf{i}}\right)$ generate $\mathscr{L}_{\mathbf{d}}$ as a $\mathbf{Z}\left[v^{-1}\right]$ module; their images $\tau\left(u\left(x_{0}\right)\right)$ form a subset $\widetilde{B[\mathbf{d}]}$ of $\mathscr{L}_{\mathbf{d}} / v^{-1} \mathscr{L}_{\mathbf{d}}$ that is independent of $\mathbf{i}$.

Theorem 8.10. (a) The restriction of $\tau: \mathscr{L}_{\mathbf{d}} \rightarrow \mathscr{L}_{\mathbf{d}} / v^{-1} \mathscr{L}_{\mathbf{d}}$ defines an isomorphism of Z-modules $\tau^{\prime}: \mathscr{L}_{\mathbf{d}} \cap \overline{\mathscr{L}}_{\mathbf{d}} \cong \mathscr{L}_{\mathbf{d}} / v^{-1} \mathscr{L}_{\mathbf{d}}$.

(b) $B[\mathbf{d}]=\widetilde{B[\mathbf{d}]}-\{0\}$ is a $\mathbf{Z}$-basis of $\mathscr{L}_{\mathbf{d}} / v^{-1} \mathscr{L}_{\mathbf{d}}$.

(c) If we set $\mathbf{B}[\mathbf{d}]=\tau^{\prime-1}(B[\mathbf{d}])$ then $\mathbf{B}[\mathbf{d}]$ is a $\mathbf{Z}$-basis of $\mathscr{L}_{\mathbf{d}} \cap \overline{\mathscr{L}}_{\mathbf{d}}$.

(d) $\mathbf{B}[\mathbf{d}]$ is a $\mathbf{Z}\left[v^{-1}\right]$-basis of $\mathscr{L}_{\mathbf{d}}$, a $\mathbf{Z}[v]$-basis of $\overline{\mathscr{L}}_{\mathbf{d}}$, an $A$-basis of $L_{\mathbf{d}, A}$ and an $A^{\prime}$-basis of $L_{\mathrm{d}}$.

(e) Each element of $\mathbf{B}[\mathbf{d}]$ is fixed by ${ }^{-}: L_{\mathbf{d}} \rightarrow L_{\mathbf{d}}$.

(f) The map $\mathscr{E} \rightarrow \mathscr{E} x_{0}$ defines a bijection $\mathbf{B}-\Psi\left(\mathbf{B}^{\prime}(\mathbf{d}) \cup \mathbf{B}^{\prime \prime}(\mathbf{d})\right) \cong \mathbf{B}[\mathbf{d}]$.

It is known that the map $\mathrm{U}^{+} / I_{\mathrm{d}} \rightarrow L_{\mathrm{d}}, u \rightarrow u x_{0}$ is an isomorphism. Using 8.2 and its proof (8.6) we see that $\mathbf{B}-\Psi\left(\mathbf{B}^{\prime}(\mathbf{d}) \cup \mathbf{B}^{\prime \prime}(\mathbf{d})\right)$ is mapped by the last isomorphism bijectively onto an $A^{\prime}$-basis $\mathscr{B}$ of $L_{\mathrm{d}}$. Using 3.2 , we see that the elements of $\mathscr{B}$ are fixed by ${ }^{-}: L_{\mathbf{d}} \rightarrow L_{\mathbf{d}}$ and that they form a $\mathbf{Z}\left[v^{-1}\right]$-basis of $\mathscr{L}_{\mathrm{d}}$, a $\mathrm{Z}[v]$-basis of $\overline{\mathscr{L}}_{\mathrm{d}}$ and a $\mathrm{Z}$-basis of $\mathscr{L}_{\mathrm{d}} \cap \overline{\mathscr{L}}_{\mathrm{d}}$; moreover, we see from 3.2 that $\tau^{\prime}$ defines a bijection $\mathscr{B} \cong B[\mathrm{~d}]$. It follows immediately that all the statements of the theorem hold with $\mathbf{B}[\mathbf{d}]=\mathscr{B}$.

8.11. We call $\mathbf{B}[\mathbf{d}]$ the canonical basis of $L_{\mathrm{d}}$. It is compatible with the decomposition of $L_{\mathrm{d}}$ into weight spaces. By specializing $v$ to 1 we obtain a natural (integral) basis in any finite-dimensional simple module of the corresponding semisimple Lie algebra.

8.12. Let $Z_{\mathbf{d}}$ be the subset of $\mathbf{N}^{\nu} \times \mathbf{N}^{\nu}$ consisting of all pairs $\mathbf{c}=\left(c_{1}, \ldots, c_{\nu}\right)$, $\mathbf{c}^{\prime}=\left(c_{1}^{\prime}, \ldots, c_{\nu}^{\prime}\right)$ such that

$$
\begin{gathered}
R_{\mathbf{i}}^{\mathrm{j}}(\mathbf{c})=\mathbf{c}^{\prime}, \\
c_{i} \leq d_{i} \quad \text { for } 1 \leq i \leq t, \\
c_{i}^{\prime} \leq d_{n-i+1} \quad \text { for } 1 \leq i \leq n-t .
\end{gathered}
$$

$\left(R_{\mathbf{i}}^{\mathbf{j}}: \mathbf{N}^{\nu} \rightarrow \mathbf{N}^{\nu}\right.$ is as in 2.6. By definition, it is a "piecewise linear" function.)

Theorem 8.13. There is a canonical bijection $\mathbf{B}[\mathbf{d}] \cong Z_{\mathbf{d}}$. Hence $\operatorname{dim} L_{\mathbf{d}}=$ $\#\left(Z_{\mathbf{d}}\right)$. 
We have

$$
\begin{aligned}
\mathbf{B}_{\mathbf{d}} & \cong \mathbf{B}-\Psi\left(\mathbf{B}^{\prime}(\mathbf{d}) \cup \mathbf{B}^{\prime \prime}(\mathbf{d})\right) \\
& \cong \mathbf{B}-\left(\mathbf{B}^{\prime}(\mathbf{d}) \cup \mathbf{B}^{\prime \prime}(\mathbf{d})\right) \\
& =\left(\mathbf{B}-\mathbf{B}^{\prime}(\mathbf{d})\right) \cap\left(\mathbf{B}-\mathbf{B}^{\prime \prime}(\mathbf{d})\right)
\end{aligned}
$$

(the first bijection is given by 8.10 (f) and the second one by $\Psi$, see 3.3 ).

The canonical bijections $8.4(\mathrm{a})$ restrict to bijections

$$
\begin{aligned}
& \mathbf{B}-\mathbf{B}^{\prime}(\mathbf{d}) \cong B_{\mathbf{i}}-\left(B_{\mathbf{i}} \cap J_{\mathbf{d}}^{\prime}\right), \\
& \mathbf{B}-\mathbf{B}^{\prime \prime}(\mathbf{d}) \cong B_{\mathbf{j}}-\left(B_{\mathbf{j}} \cap J_{\mathbf{d}}^{\prime \prime}\right) .
\end{aligned}
$$

Combining the bijections above we obtain a bijection between $\mathbf{B}_{\mathbf{d}}$ and the set of all pairs $\left(\beta^{\prime}, \beta^{\prime \prime}\right) \in B_{\mathbf{i}} \times B_{\mathbf{j}}$ such that $\beta^{\prime} \in B_{\mathbf{i}}-\left(B_{\mathbf{i}} \cap J_{\mathbf{d}}^{\prime}\right), \beta^{\prime \prime} \in B_{\mathbf{j}}-\left(B_{\mathbf{i}} \cap J_{\mathbf{d}}^{\prime \prime}\right)$, and $f\left(\beta^{\prime}\right)=\beta^{\prime \prime}$ where $f: B_{\mathbf{i}} \cong B_{\mathrm{j}}$ is obtained by composing the bijections 8.4(a). We now use the bijections $8.3(\mathrm{c}),(\mathrm{d})$ and the theorem follows.

\section{INTERSECTION COHOMOLOGY}

9.1. In this section, $F$ is an algebraic closure of a finite field $F_{q}$. We fix an orientation $\Omega$ for our Dynkin graph, and we choose $\mathbf{i} \in \mathscr{X}$ adapted to $\Omega$.

Consider the partial order on $\mathbf{N}^{\nu}$ defined by $\mathbf{c}^{\prime} \preceq \mathbf{c}^{\prime \prime}$ if $\mathbf{c}^{\prime}, \mathbf{c}^{\prime \prime}$ have the same i- homogeneity and $\mathscr{O}_{\mathbf{c}^{\prime}}$ is contained in the Zariski closure of $\mathscr{O}_{c}$ (orbits as in 4.16).

9.2. We fix a prime number $l$, invertible in $F$. Given $\mathbf{d} \in \mathbf{N}^{n}$, let $\mathscr{K}_{\mathbf{d}}$ be the vector space of $\overline{\mathbf{Q}}_{l}$-valued functions on the set of $F_{q}$-rational points of $\mathbf{E}_{\mathbf{d}}$ that are constant on the orbits of the group of $G_{\mathbf{d}}\left(F_{q}\right)$ on $\mathbf{E}_{\mathbf{d}}\left(F_{q}\right)$ (see 4.16) or, equivalently, on the set of $F_{q}$-rational points of any $G_{\mathrm{d}}$-orbit on $\mathbf{E}_{\mathbf{d}}$. (The isotropy groups of the $G_{\mathbf{d}}$ action are connected.) Let $\gamma_{c}$ be the function that equals 1 on the $F_{q}$-rational points of $\left(\mathscr{O}_{c}\right)$ and is zero elsewhere. These functions form a $\overline{\mathbf{Q}}_{l}$-basis of $\mathscr{K}_{\mathbf{d}}$.

9.3. Let $\mathbf{d}, \mathbf{d}^{\prime}, \mathbf{d}^{\prime \prime} \in \mathbf{N}^{n}$ be such that $\mathbf{d}=\mathbf{d}^{\prime}+\mathbf{d}^{\prime \prime}$. Assume given functions $f^{\prime} \in \mathscr{K}_{\mathbf{d}^{\prime}}, f^{\prime \prime} \in \mathscr{K}_{\mathbf{d}^{\prime}}$. We define a function $f=f^{\prime} \star f^{\prime \prime} \in \mathscr{K}_{\mathbf{d}}$ as follows. We consider the diagram of varieties

$$
\mathbf{E}_{\mathbf{d}^{\prime}} \times \mathbf{E}_{\mathbf{d}^{\prime \prime}} \stackrel{\beta}{\stackrel{\beta^{\prime}}{\longrightarrow}} \mathbf{E}^{\prime} \stackrel{\beta^{\prime}}{\longrightarrow} \mathbf{E}^{\prime \prime} \stackrel{\beta^{\prime \prime}}{\longrightarrow} \mathbf{E}_{\mathbf{d}}
$$

as in $6.1(a)$. We set

$$
f(e)=\sum_{e^{\prime}, e^{\prime \prime}} \frac{\#\left\{\hat{e} \mid \beta(\hat{e})=\left(e^{\prime}, e^{\prime \prime}\right), \beta^{\prime \prime}\left(\beta^{\prime}(\hat{e})\right)=e\right\}}{\#\left(G_{\mathbf{d}^{\prime}}\left(F_{q}\right)\right) \#\left(G_{\mathbf{d}^{\prime \prime}}\left(F_{q}\right)\right)} f^{\prime}\left(e^{\prime}\right) f^{\prime \prime}\left(e^{\prime \prime}\right)
$$

where $e^{\prime}, e^{\prime \prime}, e, \hat{e}$ are $F_{q}$-rational points of $\mathbf{E}_{\mathbf{d}^{\prime}}, \mathbf{E}_{\mathbf{d}^{\prime \prime}}, \mathbf{E}_{\mathbf{d}}, \mathbf{E}^{\prime}$ respectively. The operation $f^{\prime} \star f^{\prime \prime}$ (convolution) defines an associative algebra structure on $\mathscr{K}=$ $\bigoplus_{\mathbf{d}} \mathscr{K}_{\mathbf{d}}$. Let us identify the fields $\overline{\mathbf{Q}}_{l}=\mathbf{C}$. We have a vector space isomorphism

$$
\mathscr{K} \cong R \Omega
$$


that takes the basis element $\gamma_{c}$ to $V_{c}$ (see 4.15). Using 6.8(a) we see that this is an algebra isomorphism.

9.4. Let $\mathbf{A}$ be the group ring of $\overline{\mathbf{Q}}_{l}^{*}$ over $\mathbf{Z}$. Let $\mathbf{K}_{\mathbf{d}}$ be the free A-module with basis $\left(\gamma_{\mathbf{c}}^{\prime}\right)$ indexed by the $G_{\mathbf{d}}$-orbits $\left(\mathscr{O}_{\mathbf{c}}\right)$ on $\mathbf{E}_{\mathbf{d}}$. We may identify $\mathbf{K}_{\mathbf{d}}$ with the Grothendieck group of the category of constructible, $G_{\mathrm{d}}$ - equivariant $\overline{\mathbf{Q}}_{l}$ sheaves on $\mathbf{E}_{\mathbf{d}}$, defined over $F_{q}$; the basis element $\left(\gamma_{\mathbf{c}}^{\prime}\right)$ corresponds to the constant sheaf $\overline{\mathbf{Q}}_{l}$ on the orbit $\left(\mathscr{\mathscr { O }}_{c}\right)$, extended by 0 on the complement; the Frobenius map acts as identity on the stalks at rational points of the orbit. Let $\mathscr{P}_{\mathbf{c}}$ be the intersection cohomology complex of the closure of $\mathscr{O}_{\mathbf{c}}$ in $\mathbf{E}_{\mathbf{d}}$ (with coefficients in $\overline{\mathbf{Q}}_{l}$ ) extended by zero on the complement of that closure, with the $F_{q}$ structure such that the Frobenius map acts as identity on the stalks $\left(\cong \overline{\mathbf{Q}}_{l}\right)$ of its 0 th cohomology sheaf at rational points of the orbit $\mathscr{O}_{c}$. We associate to $\mathscr{P}_{\mathrm{c}}$ an element

$$
\tilde{\gamma_{\mathbf{c}}^{\prime}}=\sum_{\mathbf{c}^{\prime} \leq \mathbf{c}} p_{\mathbf{c}^{\prime}}^{\mathbf{c}} \gamma_{\mathbf{c}^{\prime}}^{\prime} \in \mathbf{K}_{\mathbf{d}}
$$

where $p_{\mathbf{c}^{\prime}}^{\mathbf{c}}$ is the (formal) alternating sum of the eigenvalues (in $\overline{\mathbf{Q}}_{l}^{*}$ ) of the Frobenius map on the stalks of the cohomology sheaves of $\mathscr{P}_{c}$ at any $F_{q}$ rational point of $\mathscr{O}_{\mathbf{c}^{\prime}}$. (The sum is taken in $\mathbf{A}$ rather than in $\overline{\mathbf{Q}}_{l}$.) We have $p_{\mathbf{c}}^{\mathbf{c}}=1$. We define $p_{\mathbf{c}^{\prime}}^{\mathbf{c}}=0$ whenever the condition $\mathbf{c}^{\prime} \preceq \mathbf{c}$ is not satisfied.

9.5. We now show that the convolution operation of 9.3 makes also sense in the context of derived categories.

Consider again the diagram 6.1 (a) with $\mathbf{d}, \mathbf{d}^{\prime}, \mathbf{d}^{\prime \prime} \in \mathbf{N}^{n}$ such that $\mathbf{d}=\mathbf{d}^{\prime}+\mathbf{d}^{\prime \prime}$. Assume given two orbits $\mathscr{O}_{\mathbf{c}^{\prime}} \subset \mathbf{E}_{\mathbf{d}^{\prime}}, \mathscr{O}_{\mathbf{c}^{\prime \prime}} \subset \mathbf{E}_{\mathbf{d}^{\prime \prime}}$. Let $\mathscr{P}^{\prime}=\mathscr{P}_{\mathbf{c}^{\prime}}, \quad \mathscr{P}^{\prime \prime}=\mathscr{P}_{\mathbf{c}^{\prime \prime}}$ be as in 9.4. Then $\mathscr{P}^{\prime}$ (resp. $\mathscr{P}^{\prime \prime}$ ) is a $G_{\mathrm{d}^{\prime}}$ (resp. $G_{\mathrm{d}^{\prime \prime}}$ ) equivariant complex; hence using 6.1,6.2, there is a well-defined complex of sheaves $\widetilde{P}$ on $\mathbf{E}^{\prime \prime}$ such that

$$
\beta^{\prime *}(\widetilde{P})=\beta^{*}\left(\mathscr{P}^{\prime} \otimes \mathscr{P}^{\prime \prime}\right) .
$$

( $\widetilde{P}$ is (up to shift) a simple perverse sheaf on $\mathbf{E}^{\prime \prime}$ defined over $F_{q}$.) We define a complex of sheaves on $\mathbf{E}_{\mathbf{d}}$ (defined over $F_{q}$ ) by

$$
\mathscr{P}^{\prime} \star \mathscr{P}^{\prime \prime}=\beta_{*}^{\prime \prime} \tilde{P} \text {. }
$$

As in 9.4, taking formal alternating sums of eigenvalues of Frobenius on stalks, we associate to $\mathscr{P}^{\prime}, \mathscr{P}^{\prime \prime}, \mathscr{P}^{\prime} \star \mathscr{P}^{\prime \prime}$ elements $p^{\prime}, p^{\prime \prime}, p^{\prime} \star p^{\prime \prime}$ in $\mathbf{K}_{\mathrm{d}^{\prime}}, \mathbf{K}_{\mathrm{d}^{\prime \prime}}, \mathbf{K}_{\mathrm{d}^{\prime \prime}}$ respectively. It is easy to see that $p^{\prime}, p^{\prime \prime} \rightarrow p^{\prime} \star p^{\prime \prime}$ defines a A-bilinear pairing $\star: \mathbf{K}_{\mathbf{d}^{\prime}} \times \mathbf{K}_{\mathbf{d}^{\prime \prime}} \rightarrow \mathbf{K}_{\mathbf{d}}$ and hence a A-bilinear pairing $\star: \mathbf{K} \times \mathbf{K} \rightarrow \mathbf{K}$ where $\mathbf{K}=\sum_{\mathbf{d}} \mathbf{K}_{\mathbf{d}}$. We thus obtain an associative algebra structure on $\mathbf{K}$.

Since $\beta^{\prime \prime}$ is proper, $\mathscr{P}^{\prime} \star \mathscr{P}^{\prime \prime}$ above is a "pure complex." From the theory of pure complexes [BBD, 5.4.4, 5.3.9], it follows that for any $G_{\mathrm{d}}$ orbit $\mathscr{O}_{\mathbf{c}}$ in $\mathbf{E}_{\mathrm{d}}$ there exists a graded $\overline{\mathbf{Q}}_{l}$-vector space $\bigoplus_{s} \mathscr{V}_{c}^{s}$ with Frobenius action such that 


$$
p^{\prime} \star p^{\prime \prime}=\tilde{\gamma}_{\mathbf{c}^{\prime}}^{\prime} \tilde{\gamma}_{\mathbf{c}^{\prime \prime}}^{\prime \prime}=\sum_{\mathbf{c}} G\left(\mathbf{c}, \mathbf{c}^{\prime}, \mathbf{c}^{\prime \prime}\right) \tilde{\gamma}_{\mathbf{c}}^{\prime}
$$

where $G\left(\mathbf{c}, \mathbf{c}^{\prime}, \mathbf{c}^{\prime \prime}\right) \in \mathbf{A}$ is the (formal) alternating sum over $s$ of the eigenvalues of Frobenius on $\mathscr{V}_{\mathbf{c}}^{s}$ and

(b) the eigenvalues of Frobenius on $\mathscr{V}_{c}^{s}$ are algebraic numbers all of whose complex conjugates have absolute value $q^{s / 2}$.

9.6. There is a natural group homomorphism $l_{q}: \mathbf{K} \rightarrow \mathscr{K}$ that takes the basis element $\gamma_{c}^{\prime}$ to $\gamma_{c}$ and on coefficients is given by the ring homomorphism $\mathbf{A} \rightarrow$ $\overline{\mathbf{Q}}_{l}$ that is the identity on $\overline{\mathbf{Q}}_{l}^{*}$. It follows easily from the definitions that $l_{q}$ is a ring homomorphism.

If we replace $q$ by a power $q^{s}, \mathbf{K}, \mathscr{K}$ become $\mathbf{K}_{s}, \mathscr{K}_{s}$ and we have as above a ring homomorphism $l_{q^{s}}: \mathbf{K}_{s} \rightarrow \mathscr{K}_{s}$. On the other hand, we have a ring homomorphism $\mathbf{K} \rightarrow \mathbf{K}_{s}$ that is the identity on each basis element and on coefficients is given by the ring homomorphism $\mathbf{A} \rightarrow \mathbf{A}$ defined by raising to the $s$ th power on $\overline{\mathbf{Q}}_{l}^{*}$. Composing this with $l_{q^{s}}$ we obtain a ring homomorphism $\mathbf{K} \rightarrow \mathscr{K}_{s}$. Thus we have infinitely many homomorphisms of $\mathbf{K}$ to the various $\mathscr{K}_{s}(s=1,2, \ldots)$. Using these, one can deduce various properties of $\mathbf{K}$ from the corresponding properties of $\mathscr{K}$.

9.7. Let $U_{\mathrm{A}}, U_{\mathrm{A}}^{+}$be the A-algebras obtained from $U, U^{+}$by tensoring with $\mathbf{A}$ over $A$, where $\mathbf{A}$ is regarded as a $A$-algebra via the imbedding $A \subset \mathbf{A}$ that takes $v$ to $q^{1 / 2} \in \overline{\mathbf{Q}}_{l}^{*}$. Consider the ring homomorphism ${ }^{-}: \mathbf{A} \rightarrow \mathbf{A}$ that takes each element in $\overline{\mathbf{Q}}_{l}^{*}$ to its inverse. The imbedding $A \subset \mathbf{A}$ considered above is compatible with the involutions ${ }^{-}: A \rightarrow A,^{-}: \mathbf{A} \rightarrow \mathbf{A}$. Hence the ring involutions ${ }^{-}: U \rightarrow U,^{-}: U^{+} \rightarrow U^{+}$extend to ring involutions ${ }^{-}: U_{\mathbf{A}} \rightarrow U_{\mathbf{A}},{ }^{-}: U_{\mathbf{A}}^{+} \rightarrow U_{\mathbf{A}}^{+}(u \otimes a \rightarrow \bar{u} \otimes \bar{a})$. (On the other hand, ${ }^{-}$is not well defined on $U_{q}, U_{q}^{+}$, see 5.5.)

For $j \in[1, n]$, let $\gamma_{j}^{\prime}$ be the unique element $\gamma_{c}^{\prime}$ in the standard basis of $\mathbf{K}_{\mathbf{d}}$ where d has the $j$ th coordinate equal to 1 and the other coordinates equal to 0 .

Proposition 9.8. (a) The elements $\gamma_{j}^{\prime}(1 \leq j \leq n)$ generate $\mathbf{K}$ as an A-algebra.

(b) Let $\mathbf{z}=\left(z_{1}, \ldots, z_{n}\right) \in \mathbf{Z}^{n}$ be as in 5.5. There is a unique imbedding of A-algebras $\boldsymbol{\Gamma}: \mathbf{K} \hookrightarrow U_{\mathbf{A}}$ such that

$$
\Gamma\left(\gamma_{j}^{\prime}\right)=K_{j}^{-z_{j}} E_{j}
$$

for all $j \in[1, n]$.

(c) Let

$$
S(\mathbf{d})=\sum_{i}\left(d_{i}^{2}-d_{i}\right)\left(2 z_{. i}-1\right) / 2-\sum_{i \rightarrow j} d_{i} d_{j} z_{i} .
$$

There is a well-defined A-linear map $\Theta: \mathbf{K}_{\mathbf{d}} \rightarrow U_{\mathbf{A}}^{+}$such that

$$
\boldsymbol{\Gamma}(\boldsymbol{\xi})=v^{S(\mathbf{d})} K(\mathbf{d}) \Theta(\xi),
$$

for all $\xi \in \mathbf{K}_{\mathbf{d}}$, where $K(\mathbf{d})=K_{1}^{-z_{1} d_{1}} \cdots K_{n}^{-z_{n} d_{n}}$. 
(d) If c has i-homogeneity $\mathbf{d}$, then

$$
\boldsymbol{\Gamma}\left(\gamma_{\mathbf{c}}^{\prime}\right)=v^{-\delta_{\mathbf{c}}+r_{\mathbf{d}}} K(\mathbf{d}) E_{\mathbf{i}}^{\mathbf{c}} \in U_{\mathbf{A}}
$$

where

$$
\begin{gathered}
K(\mathbf{d})=K_{1}^{-z_{1} d_{1}} \cdots K_{n}^{-z_{n} d_{n}}, \\
r_{\mathbf{d}}=\sum_{i} d_{i}\left(d_{i}-1\right)\left(2 z_{i}-1\right) / 2-\sum_{i \rightarrow j} d_{i} d_{j} z_{j}
\end{gathered}
$$

and $\delta_{\mathrm{c}}$ is the codimension of the orbit $\mathscr{O}_{\mathrm{c}}$.

The analogous results for $U_{q}$ and $R \Omega$ are contained in 5.4(a), 5.8 (see 5.7) and 7.13(e). The proposition follows from those results by the method of 9.6.

9.9. Verdier duality induces a homomorphism $D: \mathbf{K}_{\mathbf{d}} \rightarrow \mathbf{K}_{\mathbf{d}}$ that is antilinear with respect to the ring homomorphism ${ }^{-}: \mathbf{A} \rightarrow \mathbf{A}$.

We have

$$
D\left(\gamma_{\mathbf{c}}^{\prime}\right)=\sum_{\mathbf{c}^{\prime} \preceq \mathbf{c}} r_{\mathbf{c}^{\prime}}^{\mathbf{c}} \gamma_{\mathbf{c}^{\prime}}^{\prime}
$$

where $r_{\mathbf{c}^{\prime}}^{\mathbf{c}} \in \mathbf{A}$ and $r_{\mathbf{c}}^{\mathbf{c}}=q^{-d(\mathbf{c})}, d(\mathbf{c})=\operatorname{dim}\left(\mathscr{O}_{\mathbf{c}}\right)$. Moreover,

$$
D\left(\tilde{\gamma}_{\mathbf{c}}^{\prime}\right)=q^{-d(\mathbf{c})} \tilde{\gamma}_{\mathbf{c}}^{\prime} \text {. }
$$

From the definition and from $6.1(\mathrm{~b}),(\mathrm{c}),(\mathrm{d})$, we see that

$$
D\left(\xi^{\prime} \star \xi^{\prime \prime}\right)=q^{m} D\left(\xi^{\prime}\right) D\left(\xi^{\prime \prime}\right)
$$

for $\xi^{\prime} \in \mathbf{K}_{\mathbf{d}^{\prime}}, \xi^{\prime \prime} \in \mathbf{K}_{\mathbf{d}^{\prime \prime}}$ where $m=-\sum_{i} d_{i}^{\prime} d_{i}^{\prime \prime}-\sum_{i \rightarrow j} d_{i}^{\prime} d_{j}^{\prime \prime}$.

Proposition 9.10. For any $\xi \in \mathbf{K}_{\mathbf{d}}$ we have

$$
\Theta(D(\xi))=\overline{\Theta(\xi)}
$$

When $\xi=\gamma_{j}^{\prime}$ is one of the algebra generators in $9.8(\mathrm{a})$, the identity (a) is obvious: we have $D(\xi)=\xi, \Theta(\xi)=E_{j}$. It is then enough to verify the following statement for general $\xi$ : if (a) holds for some $\xi^{\prime} \in \mathbf{K}_{\mathbf{d}^{\prime}}, \xi^{\prime \prime} \in \mathbf{K}_{\mathbf{d}^{\prime \prime}}$ such that $\xi=\xi^{\prime} \star \xi^{\prime \prime}$, then it also holds for $\xi$.

Applying $\Gamma$ to 9.9 (c) and using the multiplicativity of $\Gamma$ we obtain

$$
\begin{aligned}
v^{S(\mathbf{d})} K(\mathbf{d}) \Theta(D(\xi)) & =v^{2 m} v^{S\left(\mathbf{d}^{\prime}\right)} K\left(\mathbf{d}^{\prime}\right) \Theta\left(D\left(\xi^{\prime}\right)\right) v^{S\left(\mathbf{d}^{\prime \prime}\right)} K\left(\mathbf{d}^{\prime \prime}\right) \Theta\left(D\left(\xi^{\prime \prime}\right)\right) \\
& =v^{2 m+S\left(\mathbf{d}^{\prime}\right)+S\left(\mathbf{d}^{\prime \prime}\right)+g} K(\mathbf{d}) \overline{\Theta\left(\xi^{\prime}\right) \Theta\left(\xi^{\prime \prime}\right)}
\end{aligned}
$$

where

$$
g=\sum_{i, j} a_{i j} d_{i}^{\prime} d_{j}^{\prime \prime} z_{j}
$$

Hence

$$
\Theta(D(\xi))=v^{2 m+S\left(\mathbf{d}^{\prime}\right)+S\left(\mathbf{d}^{\prime \prime}\right)-S(\mathbf{d})+g} \overline{\Theta\left(\xi^{\prime}\right) \Theta\left(\xi^{\prime \prime}\right)} .
$$


Similarly, applying $\Gamma$ to $\xi=\xi^{\prime} \star \xi^{\prime \prime}$ we have

$$
\begin{aligned}
v^{S(\mathbf{d})} K(\mathbf{d}) \Theta(\xi) & =v^{S\left(\mathbf{d}^{\prime}\right)} K\left(\mathbf{d}^{\prime}\right) \Theta\left(\xi^{\prime}\right) v^{S\left(\mathbf{d}^{\prime \prime}\right)} K\left(\mathbf{d}^{\prime \prime}\right) \Theta\left(\xi^{\prime \prime}\right) \\
& =v^{S\left(\mathbf{d}^{\prime}\right)+S\left(\mathbf{d}^{\prime \prime}\right)+g} K(\mathbf{d}) \Theta\left(\xi^{\prime}\right) \Theta\left(\xi^{\prime \prime}\right) .
\end{aligned}
$$

Hence

$$
\Theta(\xi)=v^{S\left(\mathbf{d}^{\prime}\right)+S\left(\mathbf{d}^{\prime \prime}\right)+g-S(\mathbf{d})} \Theta\left(\xi^{\prime}\right) \Theta\left(\xi^{\prime \prime}\right)
$$

Applying to this ${ }^{-}$, we obtain

$$
\overline{\Theta(\xi)}=v^{-S\left(\mathbf{d}^{\prime}\right)-S\left(\mathbf{d}^{\prime \prime}\right)-g+S(\mathbf{d})} \overline{\Theta\left(\xi^{\prime}\right) \Theta\left(\xi^{\prime \prime}\right)} .
$$

Comparing this with (c), we obtain

$$
\Theta(D(\xi))=v^{2 m+2 S\left(\mathbf{d}^{\prime}\right)+2 S\left(\mathbf{d}^{\prime \prime}\right)-2 S(\mathbf{d})+2 g} \overline{\Theta(\xi)} .
$$

It remains to show

$$
S(\mathbf{d})-S\left(\mathbf{d}^{\prime}\right)-S\left(\mathbf{d}^{\prime \prime}\right)=m+g .
$$

Using the definitions, we have

$$
m+g=\sum_{i}\left(2 z_{i}-1\right)-\sum_{i \rightarrow j} d_{i}^{\prime} d_{j}^{\prime \prime} z_{j}-\sum_{j \rightarrow i} d_{i}^{\prime} d_{j}^{\prime \prime} z_{j}-\sum_{i \rightarrow j} d_{i}^{\prime} d_{j}^{\prime \prime} .
$$

We substitute here

$$
\sum_{i \rightarrow j} d_{i}^{\prime} d_{j}^{\prime \prime} z_{j}=\sum_{i \rightarrow j} d_{i}^{\prime} d_{j}^{\prime \prime}\left(z_{i}-1\right)
$$

and

and we obtain

$$
\sum_{j \rightarrow i} d_{i}^{\prime} d_{j}^{\prime \prime} z_{j}=\sum_{i \rightarrow j} d_{i}^{\prime \prime} d_{j}^{\prime} z_{i}
$$

$$
m+g=\sum_{i}\left(2 z_{i}-1\right)-\sum_{i \rightarrow j} d_{i}^{\prime} d_{j}^{\prime \prime} z_{i}-\sum_{i \rightarrow j} d_{i}^{\prime \prime} d_{j}^{\prime} z_{i} .
$$

This is clearly equal to $S(\mathbf{d})-S\left(\mathbf{d}^{\prime}\right)-S\left(\mathbf{d}^{\prime \prime}\right)$. The proposition is proved.

9.11. Recall from $7.8(\mathrm{f})$ the following identity in $U^{+}$:

$$
\overline{E_{\mathbf{i}}^{\mathbf{c}}}=\sum_{\mathbf{c}^{\prime}} \omega_{\mathbf{c}^{\prime}}^{\mathbf{c}} E_{\mathbf{i}}^{\mathbf{c}^{\prime}}
$$

where $\omega_{\mathbf{c}^{\prime}}^{\mathbf{c}} \in A$ are uniquely determined. Let $\mathscr{E}^{\mathfrak{c}} \in \mathbf{B}$ be the unique element such that

$$
\mathscr{E}^{\mathrm{c}}=\sum_{\mathbf{c}^{\prime}} \zeta_{\mathbf{c}^{\prime}}^{\mathbf{c}} E_{\mathbf{i}}^{\mathbf{c}^{\prime}}
$$

(sum over all $\mathbf{c}^{\prime}$ of $\mathbf{i}$-homogeneity $\mathbf{d}$ ) where $\zeta_{\mathbf{c}}^{\mathbf{c}}=1$,

$$
\zeta_{\mathbf{c}^{\prime}}^{\mathbf{c}} \in v^{-1} \mathbf{Z}\left[v^{-1}\right] \text { for } \mathbf{c}^{\prime} \neq \mathbf{c}
$$

(see 3.2). 
9.12. If we assume that

$$
\omega_{\mathbf{c}^{\prime}}^{\mathbf{c}} \neq 0 \Longrightarrow \mathbf{c}^{\prime} \preceq \mathbf{c}
$$

and

$$
\omega_{\mathrm{c}}^{\mathrm{c}}=1
$$

for all c, then by an argument almost identical to the one in 7.11 we see that one can solve uniquely the system of equations

$$
Z_{\mathbf{c}^{\prime}}^{\mathbf{c}}=\sum_{\mathbf{c}^{\prime \prime}: \mathbf{c}^{\prime} \preceq \mathbf{c}^{\prime \prime} \preceq \mathbf{c}} \omega_{\mathbf{c}^{\prime}}^{\mathbf{c}^{\prime \prime}} \overline{Z_{\mathbf{c}^{\prime \prime}}^{\mathbf{c}}}
$$

for all $\mathbf{c}^{\prime} \preceq \mathbf{c}$ with unknowns $Z_{\mathbf{c}^{\prime}}^{\mathbf{c}} \in \mathbf{Z}\left[v^{-1}\right], \mathbf{c}^{\prime} \preceq \mathbf{c}$, so that $Z_{\mathbf{c}}^{\mathbf{c}}=1$ for all $\mathbf{c}$ and $Z_{\mathbf{c}^{\prime}}^{\mathbf{c}} \in v^{-1} \mathbf{Z}\left[v^{-1}\right]$ for all $\mathbf{c}^{\prime} \prec \mathbf{c}$. Moreover, we have

$$
\zeta_{\mathbf{c}^{\prime}}^{\mathbf{c}}=Z_{\mathbf{c}^{\prime}}^{\mathbf{c}}
$$

if $\mathbf{c}^{\prime} \preceq \mathbf{c}$ and

$$
\zeta_{\mathbf{c}^{\prime}}^{\mathbf{c}}=0
$$

otherwise. Now (b) certainly holds (see 7.9). The assumption (a) is also satisfied, as we shall see in the following subsection. We have the following result, reminiscent of [KL2].

Theorem 9.13. (a) If $\mathscr{O}_{\mathbf{c}^{\prime}} \not \subset \overline{\mathscr{O}_{c}}$ then $\zeta_{c^{\prime}}^{c}=0$.

(b) If $\mathscr{O}_{\mathbf{c}^{\prime}} \subset \overline{\mathscr{O}_{c}}$ then

where $p_{\mathbf{c}^{\prime}}^{\mathbf{c}}$ is as in 9.4 .

$$
\zeta_{\mathbf{c}^{\prime}}^{\mathbf{c}}=v^{d\left(\mathbf{c}^{\prime}\right)-d(\mathbf{c})} p_{\mathbf{c}^{\prime}}^{\mathbf{c}}
$$

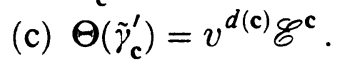

We set $\widetilde{\mathscr{E}^{c}}=\Theta\left(\tilde{\gamma}_{c}^{\prime}\right)$. From 9.10 and $9.9($ b) it follows that

$$
\overline{\widetilde{\mathscr{E}^{c}}}=q^{-d(\mathbf{c}) \widetilde{\mathscr{E}^{c}}} \text {. }
$$

From $9.8(\mathrm{c}),(\mathrm{d})$, it follows that

$$
\Theta\left(\gamma_{\mathbf{c}}^{\prime}\right)=v^{-S(\mathbf{d})-\delta_{\mathbf{c}}+r_{\mathbf{d}}} E_{\mathbf{i}}^{\mathbf{c}} \in U_{\mathbf{A}}
$$

(notation of $9.8(\mathrm{c}),(\mathrm{d}))$. Hence, applying $\Theta$ to $9.4(\mathrm{a})$, we have

$$
\widetilde{\mathscr{E}^{\mathbf{c}}}=\sum_{\mathbf{c}^{\prime}} v^{-S(\mathbf{d})-\delta_{\mathbf{c}^{\prime}+r_{\mathbf{d}}}} p_{\mathbf{c}^{\prime}}^{\mathbf{c}} E_{\mathbf{i}}^{\mathbf{c}^{\prime}} \text {. }
$$

We have

$$
\begin{aligned}
-S(\mathbf{d})-\delta_{\mathbf{c}^{\prime}}+r_{\mathbf{d}}= & -\sum_{i}\left(d_{i}^{2}-d_{i}\right)\left(2 z_{i}-1\right) / 2+\sum_{i \rightarrow j} d_{i} d_{j} z_{i} \\
& +\sum_{i} d_{i}\left(d_{i}-1\right)\left(2 z_{i}-1\right) / 2-\sum_{i \rightarrow j} d_{i} d_{j} z_{j}-\delta_{\mathbf{c}^{\prime}} \\
= & \sum_{i \rightarrow j} d_{i} d_{j}-\delta_{\mathbf{c}^{\prime}} \\
= & \operatorname{dim} \mathbf{E}_{\mathbf{d}}-\delta_{\mathbf{c}^{\prime}}=d\left(\mathbf{c}^{\prime}\right) .
\end{aligned}
$$


Thus, we can rewrite (e) in the form

$$
\widetilde{\mathscr{E}^{\mathbf{c}}}=\sum_{\mathbf{c}^{\prime}} v^{d\left(\mathbf{c}^{\prime}\right)} p_{\mathbf{c}^{\prime}}^{\mathbf{c}} E_{\mathbf{i}}^{\mathbf{c}^{\prime}}
$$

Applying - to (f) and taking into account (d) and 9.11(a), we obtain

$$
\sum_{\mathbf{c}^{\prime}} v^{-d\left(\mathbf{c}^{\prime}\right)} \overline{p_{\mathbf{c}^{\prime}}^{\mathbf{c}}} \sum_{\mathbf{c}^{\prime \prime}} \omega_{\mathbf{c}^{\prime \prime}}^{\mathbf{c}^{\prime}} E_{\mathbf{i}}^{\mathbf{c}^{\prime \prime}}=v^{-2 d(\mathbf{c})} \sum_{\mathbf{c}^{\prime \prime}} v^{d\left(\mathbf{c}^{\prime \prime}\right)} p_{\mathbf{c}^{\prime \prime}}^{\mathbf{c}} E_{\mathbf{i}}^{\mathbf{c}^{\prime \prime}} .
$$

It follows that

$$
v^{d\left(\mathbf{c}^{\prime \prime}\right)-d(\mathbf{c})} p_{\mathbf{c}^{\prime \prime}}^{\mathbf{c}}=\sum_{\mathbf{c}^{\prime}} v^{-d\left(\mathbf{c}^{\prime}\right)+d(\mathbf{c})} \overline{p_{\mathbf{c}^{\mathbf{c}}}^{\mathbf{c}}} \omega_{\mathbf{c}^{\prime \prime}}^{\mathbf{c}^{\prime}}
$$

or, if we set

$$
\mathbf{p}_{\mathbf{c}^{\prime}}^{\mathbf{c}}=v^{d\left(\mathbf{c}^{\prime}\right)-d(\mathbf{c})} p_{\mathbf{c}^{\prime}}^{\mathbf{c}}
$$

that

$$
\mathbf{p}_{\mathbf{c}^{\prime \prime}}^{\mathbf{c}}=\sum_{\mathbf{c}^{\prime}} \overline{\mathbf{p}_{\mathbf{c}^{\prime}}^{\mathbf{c}}} \omega_{\mathbf{c}^{\prime \prime}}^{\mathbf{c}^{\prime}}
$$

for all $\mathbf{c}^{\prime \prime}$. Since the matrix $\left(\mathbf{p}_{\mathbf{c}^{\prime}}^{\mathbf{c}}\right)$ is upper triangular (with respect to $\preceq$ ) with 1 on diagonal, the same must be true (by $(\mathrm{g}))$ for the matrix $\left(\omega_{\mathbf{c}^{\prime}}^{\mathrm{c}}\right)$. Thus, we see that $9.12(\mathrm{a}),(\mathrm{b})$ hold so that the results in 9.12 are applicable. As in 9.12 we can write

$$
\zeta_{\mathbf{c}^{\prime \prime}}^{\mathbf{c}}=\sum_{\mathbf{c}^{\prime}} \overline{\zeta_{\mathbf{c}^{\prime}}^{\mathbf{c}}} \omega_{\mathbf{c}^{\prime \prime}}^{\mathbf{c}^{\prime}}
$$

for all $\mathbf{c}^{\prime \prime}$. Substracting this from $(\mathrm{g})$, we obtain

$$
\mathbf{p}_{\mathbf{c}^{\prime \prime}}^{\mathbf{c}}-\zeta_{\mathbf{c}^{\prime \prime}}^{\mathbf{c}}=\sum_{\mathbf{c}^{\prime}}\left(\overline{\mathbf{p}_{\mathbf{c}^{\prime}}^{\mathbf{c}}}-\overline{\zeta_{\mathbf{c}^{\prime}}^{\mathbf{c}}}\right) \omega_{\mathbf{c}^{\prime \prime}}^{\mathbf{c}^{\prime}}
$$

for all $\mathbf{c}^{\prime \prime}$. Let $\mu_{\mathbf{c}^{\prime}}^{\mathbf{c}}=\mathbf{p}_{\mathbf{c}^{\prime}}^{\mathbf{c}}-\zeta_{\mathbf{c}^{\prime}}^{\mathbf{c}}$. Since $\mathbf{p}_{\mathbf{c}^{\prime}}^{\mathbf{c}}, \zeta_{\mathbf{c}^{\prime}}^{\mathbf{c}}, \omega_{\mathbf{c}^{\prime}}^{\mathbf{c}}$ vanish unless $\mathbf{c}^{\prime} \preceq \mathbf{c}$, and $\omega_{\mathrm{c}}^{\mathrm{c}}=1$ (see 9.12) we can deduce from $(\mathrm{h})$ that

$$
\mu_{\mathbf{c}^{\prime \prime}}^{\mathbf{c}}-\overline{\mu_{\mathbf{c}^{\prime \prime}}^{\mathbf{c}}}=\sum_{\mathbf{c}^{\prime}: \mathbf{c}^{\prime \prime} \prec \mathbf{c}^{\prime} \preceq \mathbf{c}} \overline{\mu_{\mathbf{c}^{\prime}}^{\mathbf{c}}} \omega_{\mathbf{c}^{\prime \prime}}^{\mathbf{c}^{\prime}}
$$

for all $\mathbf{c}^{\prime \prime} \preceq \mathbf{c}$.

We shall prove that

$$
\mu_{\mathrm{c}^{\prime \prime}}^{\mathrm{c}}=0
$$

for all $\mathbf{c}^{\prime \prime} \preceq \mathbf{c}$ by induction on $d(\mathbf{c})-d\left(\mathbf{c}^{\prime \prime}\right) \geq 0$. If $\mathbf{c}=\mathbf{c}^{\prime \prime}$, then $\mathbf{p}_{\mathbf{c}^{\prime \prime}}^{\mathbf{c}}=1, \zeta_{\mathbf{c}^{\prime \prime}}^{\mathbf{c}}=$ $1, \mu_{\mathbf{c}^{\prime \prime}}^{\mathbf{c}}=0$. Assume now that $\mathbf{c}^{\prime \prime} \prec \mathbf{c}$. We may assume that $\mu_{\mathbf{c}^{\prime}}^{\mathbf{c}}=0$ for all $\mathbf{c}^{\prime}$ such that $\mathbf{c}^{\prime \prime} \prec \mathbf{c}^{\prime} \preceq \mathbf{c}$. Then the right-hand side of $(\mathrm{e})$ is zero and therefore its left-hand side is also zero:

$$
\mu_{\mathbf{c}^{\prime \prime}}^{\mathrm{c}}=\overline{\mu_{\mathbf{c}^{\prime \prime}}^{\mathrm{c}}}
$$


By Gabber's purity theorem [BBD, 5.3.4], $p_{\mathbf{c}^{\prime \prime}}^{\mathbf{c}} \in \mathbf{A}$ is a (formal) $\mathbf{Z}$-linear combination of elements of $\overline{\mathbf{Q}}_{l}^{*}$ that are algebraic numbers all of whose complex conjugates have absolute value $\leq q^{\left(d(\mathbf{c})-d\left(\mathbf{c}^{\prime \prime}\right)-1\right) / 2}$. Using this, together with 9.11(c) and the definition of $\mathbf{p}_{\mathbf{c}^{\prime \prime}}^{\mathbf{c}}$, we see that $\mu_{\mathbf{c}^{\prime \prime}}^{\mathbf{c}} \in \mathbf{A}$ is a (formal) $\mathbf{Z}$-linear combination of elements of $\overline{\mathbf{Q}}_{l}^{*}$ that are algebraic numbers all of whose complex conjugates have absolute value $\leq q^{-1 / 2}$. This is compatible with $(\mathrm{k})$ only if both sides of $(k)$ are zero. Thus, $(j)$ is proved. The theorem follows.

\section{Purity}

10.1. We preserve the setup of 9.1. We also fix $\mathbf{c} \in \mathbf{N}^{\nu}$ of i-homogeneity $\mathbf{d}$. For any $k \in[1, \nu]$ we denote by $\mathbf{c}_{k}$ the element of $\mathbf{N}^{\nu}$ whose coordinates are zero except for the $k$-coordinate that is the same as the $k$ th coordinate of $\mathbf{c}$. Let $\mathbf{d}^{k} \in \mathbf{N}^{n}$ be the i-homogeneity of $\mathbf{c}_{k}$. We have $\mathbf{V}_{\mathbf{c}}=\bigoplus_{k} \mathbf{V}_{\mathbf{c}_{k}}$ compatibly with the module structures. We denote by $V_{\mathbf{c}, i}$ the $i$-component of $\mathbf{V}_{\mathbf{c}}$. We identify $\bigoplus_{i \rightarrow j} \operatorname{Hom}\left(V_{\mathbf{c}, i}, V_{\mathbf{c}, j}\right)$ with $\mathbf{E}_{\mathbf{d}}$ by choosing bases of each $V_{\mathbf{c}, i}$. The module structure of $\mathbf{V}_{\mathbf{c}}$ corresponds to a particular element $f=\left(f_{i j}\right) \in \mathbf{E}_{\mathbf{d}}$.

Given two modules $\mathbf{V}, \mathbf{V}^{\prime}$ in $M \Omega$ we denote $H\left(\mathbf{V}, \mathbf{v}^{\prime}\right)=\bigoplus_{i} \operatorname{Hom}\left(V_{i}, V_{i}^{\prime}\right)$. Let $H_{0}\left(\mathbf{V}, \mathbf{V}^{\prime}\right)$ be the vector space consisting of all element- $\cap f H\left(\mathbf{V}, \mathbf{V}^{\prime}\right)$ that are morphisms in $M \Omega$.

Lemma 10.2. (a) We have

$$
\operatorname{dim} H_{0}\left(\mathbf{V}_{\mathbf{c}}, \mathbf{V}_{\mathbf{c}}\right)=-\sum_{h \leq k ; i \rightarrow j} d_{i}^{h} d_{j}^{k}+\sum_{h \geq k ; i} d_{i}^{h} d_{i}^{k}
$$

(b) If $h>k$ then

$$
H_{0}\left(\mathbf{V}_{\mathbf{c}_{h}}, \mathbf{V}_{\mathbf{c}_{k}}\right)=0
$$

(c) If $h \leq k$, then

$$
\operatorname{dim} H_{0}\left(\mathbf{V}_{\mathbf{c}_{h}}, \mathbf{V}_{\mathbf{c}_{k}}\right)=-\sum_{i \rightarrow j} d_{i}^{h} d_{j}^{k}+\sum_{i} d_{i}^{h} d_{i}^{k}
$$

We define a linear map

$$
H\left(\mathbf{V}_{\mathbf{c}}, \mathbf{V}_{\mathbf{c}}\right) / H_{0}\left(\mathbf{V}_{\mathbf{c}}, \mathbf{V}_{\mathbf{c}}\right) \rightarrow \mathbf{E}_{\mathbf{d}}
$$

as follows. To an element $\xi \in H\left(\mathbf{V}_{\mathbf{c}}, \mathbf{V}_{\mathbf{c}}\right)$ that restricts to $\xi_{i}: \mathbf{V}_{\mathbf{c}, i} \rightarrow \mathbf{V}_{\mathbf{c}, i}$ for any $i$, we associate the element $\left(\phi_{i j}\right) \in \mathbf{E}_{\mathbf{d}}$ given by $\phi_{i j}=\xi_{j} f_{i j}-f_{i j} \dot{\xi}_{i}$ for all $i \rightarrow j$. This clearly factors through a map (d). It is also clear that (d) is injective.

Let $\mathscr{T}$ be the image of the map (d). It is easy to see that $f+\mathscr{T}$ is exactly the tangent space at $f$ to the $G_{\mathbf{d}}$-orbit in $\mathbf{E}_{\mathbf{d}}$ passing through $f$. Hence $\operatorname{dim} \mathscr{T}$ (or, equivalently, $\operatorname{dim} H\left(\mathbf{V}_{\mathbf{c}}, \mathbf{V}_{\mathbf{c}}\right) / H_{0}\left(\mathbf{V}_{\mathbf{c}}, \mathbf{V}_{\mathbf{c}}\right)$ ) is equal to the dimension of that orbit, which is given by 6.6 .

We have obviously $\operatorname{dim} H\left(\mathbf{V}_{\mathbf{c}}, \mathbf{V}_{\mathbf{c}}\right)=\sum_{h, k, i} d_{i}^{h} d_{i}^{k}$ and (a) follows. 
Now (b) is the same as 4.9(c). Next assume that $h<k$. Let

$$
\begin{gathered}
D_{1}=\operatorname{dim} H_{0}\left(\mathbf{V}_{\mathbf{c}_{h}}, \mathbf{V}_{\mathbf{c}_{k}}\right), \quad D_{2}=\operatorname{dim} H_{0}\left(\mathbf{V}_{\mathbf{c}_{h}}, \mathbf{V}_{\mathbf{c}_{h}}\right), \\
D_{3}=\operatorname{dim} H_{0}\left(\mathbf{V}_{\mathbf{c}_{k}}, \mathbf{v}_{\mathbf{c}_{k}}\right), \quad D_{4}=\operatorname{dim} H_{0}\left(\mathbf{V}_{\mathbf{c}_{h}} \oplus \mathbf{V}_{\mathbf{c}_{k}}, \mathbf{v}_{\mathbf{c}_{h}} \oplus \mathbf{V}_{\mathbf{c}_{k}}\right) .
\end{gathered}
$$

Then, by (b), we have $D_{4}=D_{1}+D_{2}+D_{3}$. Moreover, $D_{2}, D_{3}, D_{4}$ are given by formulas that are special cases of (a). Hence we can find $D_{1}=D_{4}-D_{2}-D_{3}$ and we see that (c) holds in this case. Finally, if $h=k$, then (c) is a special case of (a). The lemma is proved.

10.3. We have a direct sum decomposition $\mathbf{E}_{\mathbf{d}}=\mathbf{E}_{\mathbf{d}}^{h, k}$ indexed by $(h, k) \in$ $[1, \nu] \times[1, \nu]$ where

$$
\mathbf{E}_{\mathbf{d}}^{h, k}=\bigoplus_{i \rightarrow j} \operatorname{Hom}\left(V_{\mathbf{c}_{h}, i}, V_{\mathbf{c}_{k}, j}\right) .
$$

Lemma 10.4. $\mathscr{T}$ (see 10.2) contains the subspace $\bigoplus_{h \leq k} \mathbf{E}_{\mathbf{d}}^{h, k}$ of $\mathbf{E}_{\mathbf{d}}$.

It is enough to show that for any $h \leq k$, the map 10.2(d) restricts to an isomorphism

$$
H\left(\mathbf{V}_{\mathbf{c}_{h}}, \mathbf{V}_{\mathbf{c}_{k}}\right) / H_{0}\left(\mathbf{V}_{\mathbf{c}_{h}}, \mathbf{V}_{\mathbf{c}_{k}}\right) \rightarrow \mathbf{E}_{\mathbf{d}}^{h, k}
$$

The map (a) is certainly injective, since $10.2(\mathrm{~d})$ is injective. Using $10.2(\mathrm{c})$, we see that the first space in (a) has dimension $\sum_{i \rightarrow j} d_{i}^{h} d_{j}^{k}$, which is clearly equal to $\operatorname{dim} \mathbf{E}_{\mathbf{d}}^{h, k}$. Hence (a) must be an isomorphism and the lemma is proved.

10.5. For each $t \in F^{*}$, we define an element $\lambda(t) \in G_{\mathbf{d}}$ by the requirement that its component in $G L\left(V_{\mathbf{c}, i}\right)$ acts on the summand $V_{\mathbf{c}_{k}, i}$ as $t^{k}$ times identity.

(a) In the action of $G_{\mathbf{d}}$ on $\mathbf{E}_{\mathbf{d}}, \lambda(t)$ acts on the summand $\mathbf{E}_{\mathbf{d}}^{h, k}$ as $t^{k-h}$ times identity.

Note that $f$ is contained in $\bigoplus_{k} \mathbf{E}_{\mathbf{d}}^{k, k}$; hence it is fixed by $\lambda(t)$. Now $\mathscr{T}$ is stable under the 1-parameter group $\lambda$ since it is the sum of its intersections with the various $\mathbf{E}_{\mathbf{d}}^{h, k}$ that are stable. Hence there exists a linear subspace $\mathscr{T}^{\prime}$ that is a complement of $\mathscr{T}$ in $\mathbf{E}_{\mathbf{d}}$ and is stable under the 1-parameter group $\lambda$. Note that

(b) the action of the 1-parameter group $\lambda$ on $\mathscr{T}^{\prime}$ has all weights of the form $t \rightarrow t^{s}$, for some $s<0$.

This follows from (a) and 10.3.

Proposition 10.6. Assume tinat $f$ is an $F_{q}$-rational point of the orbit $\mathscr{O}_{\mathbf{c}}$ in $\mathbf{E}_{\mathbf{d}}$. Let $\mathscr{O}_{\mathbf{c}^{\prime}}$ be an orbit whose closure contains $\mathscr{O}_{\mathbf{c}}$. Let $\mathscr{H}_{f}^{a}$ be the stalk at $f$ of the ath cohomology sheaf of the intersection cohomology complex of the closure of $\mathscr{O}_{\mathbf{c}^{\prime}}$. Then all eigenvalues of the Frobenius map on $\mathscr{H}_{f}^{a}$ are algebraic numbers all of whose complex conjugates have absolute value $q^{a / 2}$.

The proof has some similarity to a proof in [KL2]. We may assume that the result is true when $f$ is replaced by a rational point in an orbit of dimension 
stricly bigger than that of $\mathscr{O}_{c}$. Let $\mathscr{T}^{\prime}$ and $\lambda$ be as in 10.5. We may assume that they are defined over $F_{q}$. From the definition of $\mathscr{T}^{\prime}$ we see easily that $f+\mathscr{T}^{\prime}$ is a transversal slice in $\mathbf{E}$ to the $G_{\mathrm{d}}$-orbit of $f$, and from $10.5(\mathrm{~b})$ we see that $\lambda$ defines a linear contraction of that slice to $f$. Now any $G_{\mathrm{d}}$-orbit is stable under $\lambda$. Hence the intersection of $f+\mathscr{T}^{\prime}$ with the closure of $\mathscr{O}_{\mathbf{c}^{\prime}}$ is stable under $\lambda$. Consider the cohomology sheaves of the intersection complex of this intersection. The eigenvalues of Frobenius on its stalks at points other than $f$ have a property like the one asserted in the proposition, by the induction hypothesis. We only have to show that they have that property at $f$. But this follows from [KL2, 4.5(b)], where it is deduced from the hard Lefschetz theorem of Deligne.

Corollary 10.7. (a) If $a$ is odd, then $\mathscr{H}_{f}^{a}=0$.

(b) $\sum_{a} \operatorname{dim} \mathscr{H}_{f}^{2 a} v^{2 a}=v^{d\left(\mathbf{c}^{\prime}\right)-d(\mathbf{c})} \zeta_{\mathbf{c}}^{c^{\prime}}$.

(c) In particular, $v^{d\left(\mathbf{c}^{\prime}\right)-d(\mathbf{c})} \zeta_{\mathbf{c}}^{\mathbf{c}^{\prime}}$ is a polynomial in $v^{2}$ with coefficients in $\mathbf{N}$.

(d) All eigenvalues of Frobenius on $\mathscr{H}_{f}^{2 a}$ are equal to $q^{a}$.

Let $\zeta_{\mathbf{c}}^{\prime \mathbf{c}^{\prime}}=v^{d\left(\mathbf{c}^{\prime}\right)-d(\mathbf{c})} \zeta_{\mathbf{c}}^{\mathbf{c}^{\prime}}$. The equation

$$
\zeta_{\mathbf{c}}^{\mathbf{c}^{\prime}}=\sum_{\mathbf{c}^{\prime \prime}} \overline{\zeta_{\mathbf{c}^{\prime \prime}}^{\mathbf{c}^{\prime}}} \omega_{\mathbf{c}}^{\mathbf{c}^{\prime \prime}}
$$

(see 9.12) implies

$$
\zeta_{\mathbf{c}}^{\prime \mathbf{c}^{\prime}}=\sum_{\mathbf{c}^{\prime \prime}} v^{2 d\left(\mathbf{c}^{\prime}\right)-2 d\left(\mathbf{c}^{\prime \prime}\right)} \overline{\zeta_{\mathbf{c}^{\prime \prime}}^{\prime \mathbf{c}^{\prime}}} \omega_{\mathbf{c}}^{\prime \mathbf{c}^{\prime \prime}}
$$

where $\omega_{\mathbf{c}}^{\prime \mathbf{c}^{\prime \prime}}$ is in $\mathbf{Z}\left[v^{2}, v^{-2}\right]$ (see 7.14). We want to show that

$$
\zeta_{\mathbf{c}}^{\mathbf{c}^{\prime}} \in \mathbf{Z}\left[v^{2}, v^{-2}\right] \text {. }
$$

We may assume that this is known when $\mathbf{c}$ is replaced by any $\mathbf{c}^{\prime \prime}$ with $\mathbf{c} \prec \mathbf{c}^{\prime \prime} \preceq$ $\mathbf{c}^{\prime}$ and that $\mathbf{c} \prec \mathbf{c}^{\prime}$. Then the previous equation shows that

$$
\zeta_{\mathbf{c}}^{\prime \mathbf{c}^{\prime}}-v^{2 d\left(\mathbf{c}^{\prime}\right)-2 d(\mathbf{c})} \overline{\zeta_{\mathbf{c}}^{\prime \mathbf{c}^{\prime}}} \in \mathbf{Z}\left[v^{2}, v^{-2}\right]
$$

Now $\zeta_{\mathbf{c}}^{\prime \mathbf{c}^{\prime}}$ involves only powers of $v$ with exponent $\left\langle d\left(\mathbf{c}^{\prime}\right)-d(\mathbf{c})-1\right.$, while $v^{2 d\left(\mathbf{c}^{\prime}\right)-2 d(\mathbf{c})} \overline{\zeta_{\mathbf{c}}^{\prime \mathbf{c}^{\prime}}}$ involves only powers of $v$ with exponent $>d\left(\mathbf{c}^{\prime}\right)-d(\mathbf{c})+1$. This, together with (f) implies (e). From (e) and 9.13(b) we see that $p_{c}^{c^{\prime}}$ is a formal Z-linear combination (in A ) of integral powers of $q$. This fact, together with 10.6, clearly implies (a) and (d); using again 9.13 (b) we see that (b) holds also. The corollary is proved.

10.8. The previous result shows that the coefficients $p_{\mathrm{c}}^{\mathbf{c}^{\prime}}$ in the identity

$$
\tilde{\gamma}_{\mathbf{c}^{\prime}}^{\prime}=\sum_{\mathbf{c}} p_{\mathbf{c}}^{\mathbf{c}^{\prime}} \gamma_{\mathbf{c}}^{\prime}
$$


are (as elements of $\mathbf{A}$ ) formal $\mathbf{Z}$-linear combination of integral powers of $q$. On the other hand, from 5.12 we see that the structure constants of the algebra $\mathbf{K}$ with respect to the basis $\left(\gamma_{c}^{\prime}\right)$ are (as elements of $\mathbf{A}$ ) formal $\mathbf{Z}$-linear combinations of integral powers of $q$. From these two facts it follows that

(a) the structure constants $G\left(\mathbf{c}, \mathbf{c}^{\prime}, \mathbf{c}^{\prime \prime}\right) \in \mathbf{A}$ of the algebra $\mathbf{K}$ with respect to the basis $\left(\tilde{\gamma}_{\mathrm{c}}^{\prime}\right)$ are formal $\mathrm{Z}$-linear combinations of integral powers of $q$.

Here

$$
\tilde{\gamma}_{\mathbf{c}^{\prime}}^{\prime} \tilde{\gamma}_{\mathbf{c}^{\prime \prime}}^{\prime}=\sum_{\mathbf{c}} G\left(\mathbf{c}, \mathbf{c}^{\prime}, \mathbf{c}^{\prime \prime}\right) \tilde{\gamma}_{\mathbf{c}}^{\prime} .
$$

(See 9.5.) Now let $\mathscr{G}\left(\mathbf{c}, \mathbf{c}^{\prime}, \mathbf{c}^{\prime \prime}\right) \in A$ be the structure constants of the algebra $U^{+}$with respect to the basis $\mathscr{E}^{\mathrm{c}}$. Thus

$$
\mathscr{E}^{\mathbf{c}^{\prime}} \mathscr{E}^{\mathbf{c}^{\prime \prime}}=\sum_{\mathbf{c}} \mathscr{G}\left(\mathbf{c}, \mathbf{c}^{\prime}, \mathbf{c}^{\prime \prime}\right) \mathscr{E}^{\mathbf{c}}
$$

Applying the homomorphism $\Gamma$ to (b) and using 9.13(c), we have

$$
\text { (d) } v^{d\left(\mathbf{c}^{\prime}\right)+d\left(\mathbf{c}^{\prime \prime}\right)+S\left(\mathbf{d}^{\prime}\right)+S\left(\mathbf{d}^{\prime \prime}\right)} K\left(\mathbf{d}^{\prime}\right) \mathscr{E}^{\mathbf{c}^{\prime}} K\left(\mathbf{d}^{\prime \prime}\right) \mathscr{E}^{\mathbf{c}^{\prime \prime}}=\sum_{\mathbf{c}} G\left(\mathbf{c}, \mathbf{c}^{\prime}, \mathbf{c}^{\prime \prime}\right) v^{d(\mathbf{c})+S(\mathbf{d})} \mathscr{E}^{\mathbf{c}} \text {, }
$$

where $\mathbf{d}, \mathbf{d}^{\prime}, \mathbf{d}^{\prime \prime}$ are the i-homogeneities of $\mathbf{c}, \mathbf{c}^{\prime}, \mathbf{c}^{\prime \prime}$ respectively. Since

$$
\mathscr{E}^{\mathbf{c}^{\prime}} K\left(\mathbf{d}^{\prime \prime}\right)=v^{m} K\left(\mathbf{d}^{\prime \prime}\right) \mathscr{E}^{\mathbf{c}^{\prime}}
$$

with $m=\sum_{i, j} d_{i}^{\prime} d_{j}^{\prime \prime} z_{j} a_{i j}$, we see from (c) and (d) that

$$
\mathscr{G}\left(\mathbf{c}, \mathbf{c}^{\prime}, \mathbf{c}^{\prime \prime}\right)=v^{M} G\left(\mathbf{c}, \mathbf{c}^{\prime}, \mathbf{c}^{\prime \prime}\right)
$$

where

$$
M=d(\mathbf{c})-d\left(\mathbf{c}^{\prime}\right)-d\left(\mathbf{c}^{\prime \prime}\right)+S(\mathbf{d})-S\left(\mathbf{d}^{\prime}\right)-S\left(\mathbf{d}^{\prime \prime}\right)-m .
$$

With this notation, we have the following result.

Theorem 10.9. (a) Any structure constant $G\left(\mathbf{c}, \mathbf{c}^{\prime}, \mathbf{c}^{\prime \prime}\right) \in \mathbf{A}$ of the algebra $\mathbf{K}$ with respect to the basis $\left(\tilde{\gamma}_{\mathrm{c}}^{\prime}\right)$ is a formal linear combination with integer, $\geq 0$ coefficients of integral powers of $q$.

(b) Any structure constant $\mathscr{G}\left(\mathbf{c}, \mathbf{c}^{\prime}, \mathbf{c}^{\prime \prime}\right) \in A$ of the algebra $U^{+}$with respect to the basis $\mathbf{B}$ (consisting of the elements $\mathscr{E}^{\mathbf{c}}$ ) is a linear combination with integer, $\geq 0$ coefficients of powers of $v$ with exponents of a fixed parity.

(a) follows immediately by combining $10.8(a)$ with $9.5(a),(b)$. Now (b) follows from (a) and 10.8(e).

Corollary 10.10. Let $\mathscr{E} \in \mathbf{B}$, let $i \in[1, n]$ and let $N \in \mathbf{N}$. Write

$$
E_{i}^{(N)} \mathscr{E}=\sum_{\mathscr{E}^{\prime}} M_{\mathscr{E}^{\prime}} \mathscr{\mathscr { C }}^{\prime}, \quad \mathscr{E} E_{i}^{(N)}=\sum_{\mathscr{E}^{\prime}} M_{\mathscr{E}^{\prime}}^{\prime} \mathscr{E}^{\prime}
$$

(sum over $\mathscr{E}^{\prime} \in \mathbf{B}$ ) with $M_{\mathscr{E}^{\prime}}, M_{\mathscr{E}^{\prime}}^{\prime} \in A$. Then, for each $\mathscr{E}^{\prime} \in \mathbf{B}, M_{\mathscr{E}^{\prime}}$ is a linear combination with integer $\geq 0$ coefficients of powers of $v$ with exponents of a fixed parity; the same holds for $M_{\mathcal{E}^{\prime}}^{\prime}$.

Indeed, $E_{i}^{(N)} \in \mathbf{B}$. 
Corollary 10.11. The action of $E_{i}^{(N)}$ on the U-module $L_{\mathbf{d}}$ is given by a matrix (with respect to the canonical basis $\mathbf{B}[\mathbf{d}]$ ) in which any entry (in $A$ ) is a linear combination with integer $\geq 0$ coefficients of powers of $v$ with exponents of a fixed parity.

\section{THE CYCLIC QUIVER}

11.1. Consider the oriented graph with vertices $\{1,2, \ldots, n\}$ and oriented edges $n \rightarrow n-1, n-1 \rightarrow n-2, \ldots, 3 \rightarrow 2, \ldots, 2 \rightarrow 1,1 \rightarrow n$. We call it the cyclic quiver. Then we can define a category of modules $M \Omega$ associated to this quiver just as in 4.1. As in 4.2, each module in $M \Omega$ has a well-defined dimension in $\mathbf{N}^{n}$. We shall be interested in the full subcategory $M^{0} \Omega$ of $M \Omega$ consisting of modules $\left(V_{i}, f_{i j}\right)$ such that $f_{21} f_{32} \cdots f_{n-1, n-2} f_{n, n-1} f_{1 n}: V_{1} \rightarrow$ $V_{1}$ is nilpotent. (This is equivalent to the condition that $f_{32} \cdots f_{n, n-1} f_{1 n} f_{21}$ : $V_{2} \rightarrow V_{2}$ is nilpotent.) Let $k^{\prime} \leq k$ be two integers. We define a module $\mathbf{V}\left(k^{\prime}, k\right) \in M^{0} \Omega$ as follows. By definition, $\mathbf{V}\left(k^{\prime}, k\right)_{i}$ is the $F$-vector space with basis $\left\{b(i, h) \mid k^{\prime} \leq h \leq k, h \equiv i \bmod n\right\} \quad(1 \leq i \leq n)$; we define a linear map $f_{i, i-1}: \mathbf{V}\left(k^{\prime}, k\right)_{i} \rightarrow \mathbf{V}\left(k^{\prime}, k\right)_{i-1}$ by $f_{i, i-1}(b(i, h))=b(i-1, h-1)$. (Here, $i-1$ is interpreted as $n$ in the case where $i=1$ and $b(i-1, h-1)$ is interpreted as 0 if $h=k^{\prime}$.) It is clear that $\mathbf{V}\left(k^{\prime}, k\right)$ is an indecomposable module in $M^{0} \Omega$ and that $\mathbf{V}\left(k^{\prime}, k\right) \cong \mathbf{V}\left(k_{1}^{\prime}, k_{1}\right)$ if and only if $k_{1}^{\prime}=k^{\prime}+m n$, $k_{1}=k+m n$ for some integer $m$. One can also verify that any indecomposable module in $M^{0} \Omega$ is isomorphic to some $\mathbf{V}\left(k^{\prime}, k\right)$. The simple modules are $\mathbf{V}(i, i) \quad(1 \leq i \leq n)$.

11.2. We can associate to our quiver some vector spaces $\mathbf{E}_{\mathbf{d}}$ with $G_{\mathrm{d}}$-action $\left(\mathbf{d} \in \mathbf{N}^{n}\right)$, as in 4.16. Each point of $\mathbf{E}_{\mathbf{d}}$ defines a module in $M \Omega$. The points of $\mathbf{E}_{\mathrm{d}}$ for which the corresponding module is in $M^{0} \Omega$ form a closed subvariety $\mathbf{E}_{\mathbf{d}}^{0}$ of $\mathbf{E}_{\mathbf{d}}$, stable under $G_{\mathbf{d}}$. The action of $G_{\mathbf{d}}$ on $\mathbf{E}_{\mathbf{d}}^{0}$ has only finitely many orbits (called strata); they are in 1-1 correspondence with the isomorphism classes of modules in $M^{0} \Omega$ of dimension $\mathbf{d}$; hence they can be parametrized by sequences of positive integers $\left(c_{k^{\prime}, k}\right)$ that specify how many times an indecomposable module $\mathbf{V}\left(k^{\prime}, k\right)$ appears in a given module.

The following result is a common generalization of results in [L4, Z].

Theorem 11.3. The closure of any $G_{\mathbf{d}}$ orbit in $\mathbf{E}_{\mathbf{d}}^{0}$ is locally isomorphic to the closure of an affine Schubert variety of type $A_{N-1}$ where $N=d_{1}+\cdots+d_{n}$.

The proof will be given in 11.4, 11.5.

11.4. Let $\mathbf{d}=\left(d_{1}, \ldots, d_{n}\right) \in \mathbf{N}^{n}$ and let $N=d_{1}+\cdots+d_{n}$. Let $L$ be an $N$-dimensional $F((\varepsilon))$-vector space. A lattice in $L$ is a free $F[[\varepsilon]]$-submodule of rank $N$ of $L$. We fix a sequence of lattices

$$
\mathscr{L}_{1} \supset \mathscr{L}_{2} \supset \cdots \supset \mathscr{L}_{n}
$$


in $L$ such that $\mathscr{L}_{n} \supset \varepsilon \mathscr{L}_{1}, \operatorname{dim}\left(\mathscr{L}_{i} / \mathscr{L}_{i+1}\right)=d_{i}$ for $i=1, \ldots, n-1$ and $\operatorname{dim}\left(\mathscr{L}_{n} / \varepsilon \mathscr{L}_{1}\right)=d_{n}$. (Dimensions are always taken over $F$.)

Let $Z$ be the set of all sequences

$$
\mathscr{M}_{1} \supset \mathscr{M}_{2} \supset \cdots \supset \mathscr{M}_{n}
$$

of lattices in $L$ such that $\mathscr{M}_{n} \supset \varepsilon \mathscr{M}_{1}, \mathscr{M}_{i} \subset \mathscr{L}_{i}$ for all $i$ and $\operatorname{dim} \mathscr{L}_{i} / \mathscr{M}_{i}=d_{i}$ for all $i$.

For example, the sequence $\mathscr{L}_{2} \supset \mathscr{L}_{3} \supset \cdots \supset \mathscr{L}_{n} \supset \varepsilon \mathscr{L}_{1}$ belongs to $Z$. It is clear that $Z$ is in a natural way a projective variety over $F$. We fix an $F$-subspace $P$ of $\mathscr{L}_{1}$ with a direct sum decomposition $P=\bigoplus_{i=1}^{n} P_{i}$ such that $\operatorname{dim} P_{i}=d_{i}, \mathscr{L}_{i}=\bigoplus_{j=i}^{n} P_{j} \oplus\left(\bigoplus_{j \in[1, n], h \geq 1} \varepsilon^{h} P_{j}\right)$ for $i \in[1, n]$.

Let $Z^{\prime}$ be the subset of $Z$ defined by the condition

$$
\mathscr{M}_{i} \oplus P_{i}=\mathscr{L}_{i} \text { for all } i \text {. }
$$

It is clear that $Z^{\prime}$ is an open dense subvariety of $Z$. An $F$-subspace $\mathscr{M}_{i}$ of $\mathscr{L}_{i}$ satisfies (a) precisely when it is the set of all vectors of the form

$$
\sum_{i<j \leq n} \phi_{0 j}^{i}\left(p_{0 j}\right)+\sum_{1 \leq j \leq n ; h \geq 1} \phi_{h j}^{i}\left(p_{h j}\right)-\sum_{i<j \leq n} p_{0 j}-\sum_{1 \leq j \leq n ; h \geq 1} \varepsilon^{h} p_{h j}
$$

where $p_{h j}$ runs through $P_{j}(h \geq 1$ or $h=0$ and $i<j), \phi_{h j}^{i}: P_{j} \rightarrow P_{i}$ are fixed $F$-linear maps defined for $h \geq 1$ or $h=0$ and $i<j$ and $\phi_{h j}^{i}=0$ for large $h$. (Note that the $\phi_{h j}^{i}$ are uniquely determined by $\mathscr{M}_{i}$.)

The condition $\mathscr{M}_{i} \supset \mathscr{M}_{i-1}$ (for subspaces satisfying (a)) can be written in terms of the coordinates $\phi_{h j}^{i}, \phi_{h j}^{i-1}$ as follows:

$$
\begin{gathered}
\phi_{h j}^{i-1}=\phi_{0 i}^{i-1} \phi_{h j}^{i} \quad(h \geq 1,2 \leq i \leq n), \\
\phi_{0 j}^{i-1}=\phi_{0 i}^{i-1} \phi_{0 j}^{i} \quad(2 \leq i<j \leq n) .
\end{gathered}
$$

The condition that $\mathscr{M}_{n} \supset \varepsilon \mathscr{M}_{1}$ can be written in terms of the coordinates $\phi_{h j}^{1}$, $\phi_{h j}^{n}$ as follows:

$$
\begin{array}{cc}
\phi_{h j}^{n}=\phi_{11}^{n} \phi_{h-1, j}^{1} & (h \geq 2), \\
\phi_{1 j}^{n}=\phi_{11}^{n} \phi_{0, j}^{1} & (j \geq 2), \\
\phi_{h+1,1}^{n}=\phi_{11}^{n} \phi_{h, 1}^{1} & (h \geq 1) .
\end{array}
$$

Hence we may identify $Z^{\prime}$ with the space of all sequences of linear maps $\phi_{h j}^{i}$ : $P_{i} \rightarrow P_{j}(h \geq 1$ or $h \geq 0$ and $i<j)$ satisfying the relations (b)-(f) above. (Note that the conditions $\varepsilon \mathscr{M}_{i} \subset \mathscr{M}_{i}$ are automatically satisfied; they follow from the conditions $\mathscr{M}_{1} \supset \mathscr{M}_{2} \supset \cdots \supset \mathscr{M}_{n} \supset \varepsilon \mathscr{M}_{1}$. .) From (b) we have

$$
\phi_{h j}^{i}=\phi_{0, i+1}^{i} \phi_{0, i+2}^{i+1} \cdots \phi_{0 n}^{n-1} \phi_{h j}^{n} \quad(h \geq 1) \text {. }
$$


From (c) we have

$$
\phi_{0 j}^{i}=\phi_{0, i+1}^{i} \phi_{0, i+2}^{i+1} \cdots \phi_{0 j}^{j-1} \quad(1 \leq i<j \leq n) .
$$

Note that $(b),(c)$ are equivalent to $\left(b^{\prime}\right),\left(c^{\prime}\right)$. From $(d)$ and $\left(b^{\prime}\right)$ we have

$$
\phi_{h j}^{n}=\phi_{11}^{n} \phi_{02}^{1} \phi_{03}^{2} \cdots \phi_{0 n}^{n-1} \phi_{h-1, j}^{n} \quad(h \geq 2) \text {. }
$$

Hence (d) may replaced by the condition

$$
\phi_{h j}^{n}=\left(\phi_{11}^{n} \phi_{02}^{1} \phi_{03}^{2} \cdots \phi_{0 n}^{n-1}\right)^{h-1} \phi_{i j}^{n} \quad(h \geq 1) .
$$

Similarly, (e) may be replaced by the condition

$$
\phi_{1 j}^{n}=\phi_{11}^{n} \phi_{02}^{1} \phi_{03}^{2} \cdots \phi_{0 j}^{j-1} \quad(j \geq 2) \text {. }
$$

Thus all variables can be expressed in terms of

$$
\phi_{11}^{n}, \phi_{02}^{1}, \phi_{03}^{2}, \ldots, \phi_{0 n}^{n-1}
$$

and the equations ( $f$ ) are in terms of these variables the identities expressing that $\left(\phi_{11}^{n} \phi_{02}^{1} \phi_{03}^{2} \cdots \phi_{0 n}^{n-1}\right)^{h} \phi_{11}^{n}$ is equal to itself. Thus (f) is a consequence of (b)-(e). Note that

$$
\phi_{h 1}^{n}=\left(\phi_{11}^{n} \phi_{02}^{1} \phi_{03}^{2} \cdots \phi_{0 n}^{n-1}\right)^{h-1} \phi_{11}^{n} \quad(h \geq 1) .
$$

For large $h$ we have $\phi_{h 1}^{n}=0$, and hence from (g) we see that the right-hand side of ( $\mathrm{g}$ ) is 0 for large $(\mathrm{h})$; it follows that $\phi_{11}^{n} \phi_{02}^{1} \phi_{03}^{2} \cdots \phi_{0 n}^{n-1}: P_{n} \rightarrow P_{n}$ is nilpotent. We have thus defined an isomorphism of algebraic varieties between $Z^{\prime}$ and the set of sequences

$$
\phi_{02}^{1}, \phi_{03}^{2}, \ldots, \phi_{0 n}^{n-1}, \phi_{11}^{n}
$$

of linear maps $P_{2} \rightarrow P_{1}, P_{3} \rightarrow P_{2}, \ldots, P_{n} \rightarrow P_{n-1}, P_{1} \rightarrow P_{n}$ respectively such that $\phi_{11}^{n} \phi_{02}^{1} \phi_{03}^{2} \cdots \phi_{0 n}^{n-1}: P_{n} \rightarrow P_{n}$ is nilpotent. But this set of sequences may be clearly identified with $\mathbf{E}_{\mathbf{d}}^{0}$. Hence we have defined an isomorphism

$$
Z^{\prime} \cong \mathbf{E}_{\mathbf{d}}^{0} \text {. }
$$

11.5. The group $\mathscr{G}$ of all $F((\varepsilon))$-linear isomorphisms $L \rightarrow L$ that map each $\mathscr{L}_{i}$ onto itself acts naturally on $Z$. It has finitely many orbits; the closure of any orbit is an affine Schubert variety. We now give an explicit description of the orbits.

If $\mathscr{M}_{1} \supset \mathscr{M}_{2} \supset \cdots \supset \mathscr{M}_{n}$ is a point of $Z$, we consider the linear maps induced by inclusions:

$$
\mathscr{L}_{1} / \mathscr{M}_{1} \leftarrow \mathscr{L}_{2} / \mathscr{M}_{2} \leftarrow \cdots \leftarrow \mathscr{L}_{n} / \mathscr{M}_{n}
$$

and the linear map $\mathscr{L}_{1} / \mathscr{M}_{1} \rightarrow \mathscr{L}_{n} / \mathscr{M}_{n}$ induced by multiplication by $\varepsilon$. The collection of these maps clearly defines a module in $M^{0} \Omega$. This gives a map from $Z$ to the set of isomorphism classes of modules in $M^{0} \Omega$ of dimension d. The fibres of this map are called the strata of $Z$. They coincide with the orbits of $\mathscr{G}$ on $Z$. It is clear that the intersections of the strata of $Z$ with $Z^{\prime}$ correspond under $11.4(\mathrm{~h})$ to the strata of $\mathbf{E}_{\mathbf{d}}^{0}$ described in 11.2. Since $Z^{\prime}$ is open, dense in $Z$, we see that 11.3 follows. 
Now using [KL2] we deduce

Corollary 11.6. The local intersection cohomology of the closure of any stratum of $\mathbf{E}_{\mathbf{d}}^{0}$ vanishes in odd degrees. Its Poincaré polynomials are given by some of the polynomials of [KL1] for an affine Weyl group of type $A_{N-1}$.

11.7. Let $E$ be an $N$-dimensional complex vector space with a given semisimple automorphism $s$. Fix a nonzero complex number $\zeta$. Let $P$ be the vector space of all endomorphisms $\xi: E \rightarrow E$ such that $s \xi=\zeta \xi s$. Let $P_{0}$ be the set of all elements of $P$ that are nilpotent. The centralizer of $s$ in $G L(E)$ acts on $P_{0}$ with finitely many orbits (strata). If $\zeta$ is of infinite order, the strata are known to enter naturally in the representation theory of the affine Hecke algebra with parameter $\zeta$ (Deligne-Langlands conjecture). In this case, it follows from $[Z]$ that the closures of the strata are locally isomorphic to usual Schubert varieties. In the case where $\zeta^{n}=1$, it follows from 11.3 that the closures of the strata are locally isomorphic to affine Schubert varieties. (The case $n=1$ is treated in [L4].)

\section{COMMENTS ON THE NON-SIMPLY LACED CASE.}

12.1. The discussion in $[R]$ applies also to the non-simply laced case. We present an alternative approach, which is adapted to the point of view of orbits and perverse sheaves. Assume that we are given a connected simply laced Dynkin graph and an automorphism $\tau$ of it that keeps fixed at least one vertex. Then $\tau$ acts naturally on $R^{+}$and its set of orbits may be regarded as the set of positive roots $R^{\prime+}$ of a non-simply laced root system $R^{\prime}$. We choose an orientation $\Omega$ of our Dynkin graph with the property that $\Omega$ is invariant under $\tau$. If $\mathbf{d}=\left(d_{1}, \ldots, d_{n}\right) \in \mathbf{N}^{n}$, we define $\tau(\mathbf{d})=\left(d_{\tau(1)}, \ldots, d_{\tau(n)}\right) \in \mathbf{N}^{n}$. (We identify $1, \ldots, n$ with the vertices of the Dynkin diagram.)

Consider the vector space $\mathbf{E}_{\mathbf{d}}$ (as in 4.16) over $F$, an algebraic closure of the finite field $F_{q}$. We define an isomorphism $\tau: \mathbf{E}_{\mathbf{d}} \cong \mathbf{E}_{\tau(\mathbf{d})}$ as follows: if $\left(f_{i j}\right)$ is a point of $\mathbf{E}_{\mathbf{d}}$, its image under $\tau$ is $\left(f_{i j}^{\prime}\right) \in \mathbf{d}_{\tau(\mathbf{d})}$ where $f_{i j}^{\prime}=f_{\tau(i), \tau(j)}: F^{d_{\tau(i)}} \rightarrow$ $F^{d_{\tau(j)}}$ for any $i \rightarrow j$ in $\Omega$. This isomorphism commutes with the standard Frobenius map on $\mathbf{E}_{\mathbf{d}}, \mathbf{E}_{\tau(\mathbf{d})}$ (defined by raising the obvious coordinates on these vector spaces to the $q$ th power). Similarly, we have an obvious isomorphism $\tau: G_{\mathrm{d}} \cong G_{\tau(\mathbf{d})}$.

Assume now that $\tau(\mathbf{d})=\mathbf{d}$. Then $\tau: \mathbf{E}_{\mathbf{d}} \cong \mathbf{E}_{\mathbf{d}}$ is an automorphism of finite order that commutes with the standard Frobenius map on $\mathbf{E}_{\mathbf{d}}$. Hence the composition of $\tau$ with this standard Frobenius map is a new Frobenius map $\mathrm{Fr}$ on $\mathbf{E}_{\mathbf{d}}$. Moreover, the composition of $\tau: G_{\mathbf{d}} \cong G_{\mathrm{d}}$ with the standard Frobenius map of $G_{\mathrm{d}}$ is a new Frobenius map $F r$ on $G_{\mathrm{d}}$. The natural action of $G_{\mathrm{d}}$ on $\mathbf{E}_{\mathbf{d}}$ is clearly compatible with the new Frobenius maps. We now define $\mathscr{K}_{\mathbf{d}}^{\tau}$ (as in 9.2) to be the vector space of all functions on $\mathbf{E}_{\mathbf{d}}^{F r}$ with values in $\overline{\mathbf{Q}}_{l}$ that are constant on the orbits of $G_{\mathbf{d}}^{F r}$. We define $\mathscr{K}^{\tau}$ to be the direct sum of all $\mathscr{K}_{\mathbf{d}}^{\tau}$ where $\mathbf{d}$ is subject to $\tau(\mathbf{d})=\mathbf{d}$. We can define an algebra structure on 
$\mathscr{K}^{\tau}$ just as in 9.3 (using the new Frobenius maps instead of the standard ones). Then $\mathscr{K}^{\tau}$ has a basis consisting of the characteristic functions of the various orbits which are stable under $F r$. These orbits are in 1-1 correspondence with the functions $R^{+} \rightarrow \mathbf{N}$ that are constant on the orbits of $\tau$ on $R^{+}$, hence also with the functions $R^{\prime+} \rightarrow \mathbf{N}$. From this algebra one can then recover the + part of the quantized enveloping algebra corresponding to the non-simply laced root system $R^{\prime}$ just as in 5.7.

12.2. It is likely that most of the results of this paper extend to the non-simply laced case.

\section{COMMENTS ON FOURIER TRANSFORM}

13.1. We preserve the setup of 9.1. In addition to the orientation $\Omega$, we consider another orientation $\Omega^{\prime}$. Let $\mathbf{S}$ be the set of arrows $i \rightarrow j$ of $\Omega$ such that $j \rightarrow i$ is an arrow of $\Omega^{\prime}$ and let $\mathbf{T}$ be the set of arrows $i \rightarrow j$ of $\Omega$ such that $i \rightarrow j$ is an arrow of $\Omega^{\prime}$. We shall denote by $\mathbf{E}_{\mathbf{d}}^{\prime}, \mathscr{K}_{\mathbf{d}}^{\prime}, \mathscr{K}^{\prime}$ the objects defined like $\mathbf{E}_{\mathrm{d}}, \mathscr{K}_{\mathrm{d}}, \mathscr{K}$ but in terms of $\Omega^{\prime}$ instead of $\Omega$. We define

$$
\mathbf{E}_{\mathbf{d}, \mathbf{S}}=\bigoplus_{i \rightarrow j} \operatorname{Hom}\left(F^{d_{i}}, F^{d_{j}}\right)
$$

sum over all arrows $i \rightarrow j$ in $\mathbf{S}$ and

$$
\mathbf{E}_{\mathbf{d}, \mathbf{T}}=\bigoplus_{i \rightarrow j} \operatorname{Hom}\left(F^{d_{i}}, F^{d_{j}}\right)
$$

sum over all arrows $i \rightarrow j$ in $\mathbf{T}$. We have canonical isomorphisms

$$
\mathbf{E}_{\mathbf{d}} \cong \mathbf{E}_{\mathbf{d}, \mathbf{S}} \oplus \mathbf{E}_{\mathbf{d}, \mathbf{T}}, \quad \mathbf{E}_{\mathbf{d}}^{\prime} \cong \mathbf{E}_{\mathbf{d}, \mathbf{S}}^{*} \oplus \mathbf{E}_{\mathbf{d}, \mathrm{T}},
$$

which are compatible with the natural actions of $G_{\mathrm{d}}$. Let $f$ be a function on the set of rational points of $\mathbf{E}_{\mathbf{d}}$ with values in $\overline{\mathbf{Q}}_{l}$. Its Fourier transform $\hat{f}$ is a function on the set of rational points of $\mathbf{E}_{\mathbf{d}}^{\prime}$ with values in $\overline{\mathbf{Q}}_{l}$ defined in terms of a fixed nontrivial additive character $\theta: F_{q} \rightarrow \overline{\mathbf{Q}}_{l}^{*}$ by

$$
\hat{f}\left(e_{1}+e_{2}\right)=q^{-\operatorname{dim} \mathbf{E}_{\mathrm{d} . \mathrm{s}} / 2} \sum_{e_{1}^{\prime}, e_{2}} \theta\left(e_{1}, e_{1}^{\prime}\right) f\left(e_{1}^{\prime}+e_{2}\right)
$$

where $e_{1}, e_{1}^{\prime}, e_{2}$ denote $F_{q}$-rational points of $\mathbf{E}_{\mathbf{d}, \mathbf{S}}, \mathbf{E}_{\mathbf{d}, \mathbf{S}}^{*}, \mathbf{E}_{\mathbf{d}, \mathbf{T}}$ respectively and $\left(e_{1}, e_{1}^{\prime}\right)$ is the canonical pairing $\mathbf{E}_{\mathbf{d}, \mathbf{S}} \times \mathbf{E}_{\mathbf{d}, \mathbf{S}}^{*} \rightarrow F$. The map $f \rightarrow \hat{f}$ is compatible with the action of the group of rational points of $G_{d}$; hence we get an isomorphism $\mathscr{K}_{\mathrm{d}} \cong \mathscr{K}_{\mathrm{d}}^{\prime}$. One can show that this is almost compatible with the convolution operation on $\mathscr{K}, \mathscr{K}^{\prime}$ in the sense that

$$
\widehat{f^{\prime} \star f^{\prime \prime}}=q^{\sum_{i \rightarrow j}\left(d_{i}^{\prime} d_{j}^{\prime \prime}-d_{j}^{\prime} d_{i}^{\prime \prime}\right) / 2}{\hat{f^{\prime}}}^{\prime}{\hat{f^{\prime \prime}}}^{\prime \prime}
$$

for any $f^{\prime} \in \mathscr{K}_{\mathbf{d}^{\prime}}, f^{\prime \prime} \in \mathscr{K}_{\mathbf{d}^{\prime \prime}}$, where $i \rightarrow j$ runs over all arrows in $\mathbf{S}$.

This explains why the algebra $\mathscr{K}$ is almost independent of the orientation (it is really independent of the orientation if one takes its semidirect product 
with $U_{q}^{0}$ ). Moreover, Fourier transform does not preserve the characteristic functions of orbits but it does preserve the characteristic functions of the corresponding intersection cohomology complexes. This explains why $\mathbf{U}^{+}$has many bases of PBW type, and one canonical basis $\mathbf{B}$.

\section{REFERENCES}

[BBD] A.A. Beilinson, J. Bernstein, and P. Deligne, Faisceaux pervers, Astérisque no. 100, Soc. Math. France, Paris, 1982.

[BZ] A. D. Berenstein and A.V. Zelevinskii, Tensor product multiplicities and convex polytopes in partition spaces, J. Geom. Phys. (to appear).

[BGP] I.N. Bernstein, I.M. Gelfand, and V.A. Ponomarev, Coxeter functors and Gabriel's theorem, Russian Math. Surveys 28 (1973), 17-32.

[B] N. Bourbaki, Groupes et algèbres de Lie, Hermann, Paris, 1968, Chapters 4-6.

[DK] C. De Concini and D. Kazhdan, Special bases for $S_{N}$ and $G L(n)$, Israel J. Math. 40 (1981), 275-290.

[DL] M. Dyer and G. Lusztig, Appendix to [L2], Geom. Dedicata (to appear).

[G] P. Gabriel, Unzerlegbare darstellungen I, Manuscripta Math. 6 (1972), 71-103.

[KL1] D. Kazhdan and G. Lusztig, Representations of Coxeter groups and Hecke algebras, Invent. Math. 53 (1979), 165-184.

[KL2] _ Schubert varieties and Poincaré duality, Proc. Sympos. Pure Math., vol. 36, Amer. Math. Soc., Providence, RI, 1980, pp. 185-203.

[L1] G. Lusztig, Finite dimensional Hopf algebras arising from quantized universal enveloping algebras, J. Amer. Math. Soc. 3 (1990), 257-296.

[L2] _ Quantum groups at roots of 1 , Geom. Dedicata (to appear).

[L3] Left cells in Weyl groups, Lie Group Representations. I, Lecture Notes in Math., vol. 1024, Springer-Verlag, 1983, pp. 99-111.

[L4] _ Green polynomials and singularities of unipotent classes, Adv. in Math. 42 (1981), 169-178.

[R] C. M. Ringel, Hall algebras and quantum groups, Invent. Math. (to appear).

[Z] A.V. Zelevinskii, Two remarks on graded nilpotent classes, Russian Math. Surveys 40 (1985), 249-250.

Department of Mathematics, Massachusetts Institute of Technology, Cambridge, MASSACHUSETTS 02139 